NBSIR 82-2656

\title{
Simulation of the Guard Control Station in a Computerized Site Security Monitor and Response System
}

U.S. DEPARTMENT OF COMMERCE

National Bureau of Standards

Center for Computer Systems Engineering Institute for Computer Sciences and Technology

Washington, DC 20234

January 1983

Task Code B99QMXPF

Work Unit 00065

Sponsored by:

Defense Nuclear Agency

100 Nashington, DC 20305 

SIMULATION OF THE GUARD CONTROL

STATION IN A COMPUTERIZED SITE

R. T. Moore

A. W. Holt

A. L. Koenig

A. Mink

G. Nacht

U.S. DEPARTMENT OF COMMERCE

National Bureau of Standards

Center for Computer Systems Engineering

Institute for Computer Sciences and Technology

Washington, DC 20234

January 1983

Task Code B990MXPF

Work Unit 00065

Sponsored by:

Defense Nuclear Agency

Washington, DC 20305

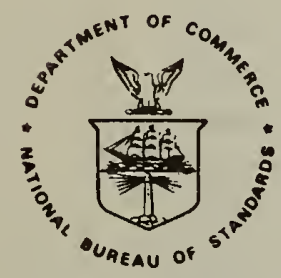

U.S. DEPARTMENT OF COMMERCE, Malcolm Baldrige, Secretary NATIONAL BUREAU OF STANDARDS, Ernest Ambler, Director 

TABLE OF CONTENTS

Page

INTRODUCTION $\ldots \ldots \ldots \ldots \ldots \ldots \ldots \ldots \ldots \ldots \ldots \ldots \ldots \ldots \ldots$

Design Objectives ........................ 4

Features Contributing to Reliability ........... 4

Features Contributing to Ease of Operation ...... 5

Purpose of the Mock-up ....................6

INITIAL DESIGN $\ldots \ldots \ldots \ldots \ldots \ldots \ldots \ldots \ldots \ldots \ldots \ldots \ldots \ldots \ldots$

SYSTEM CONFIGURATION .......................

Description of the GCS Console .............. Il

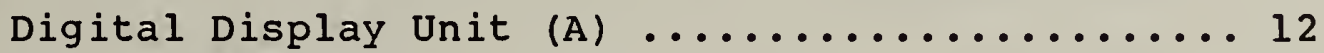

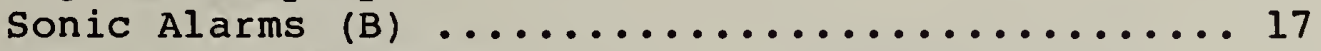

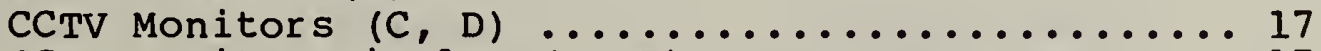

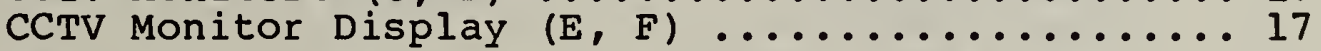

Key Port (G) .......................... 18

Voice Unit Speaker $(\mathrm{H}) \ldots \ldots \ldots \ldots \ldots \ldots \ldots . \ldots \ldots$

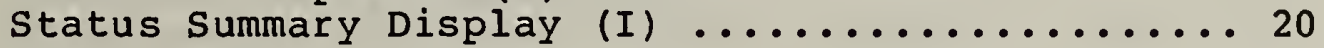

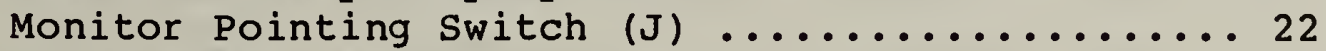

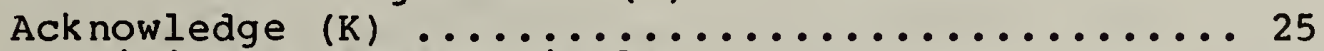

10-Digit Keypad and Display $(\mathrm{L}, \mathrm{M}) \ldots \ldots \ldots \ldots . \ldots . . \ldots 25$

Select Zone $(\mathrm{N}) \ldots \ldots \ldots \ldots \ldots \ldots \ldots \ldots \ldots . \ldots \ldots$

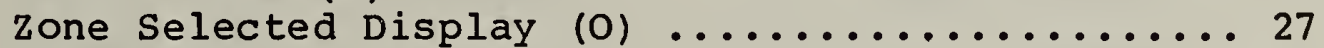

CCTV Controls $(\mathrm{P}, \mathrm{Q}, \mathrm{R}) \ldots \ldots \ldots \ldots \ldots \ldots \ldots \ldots . \ldots \ldots$

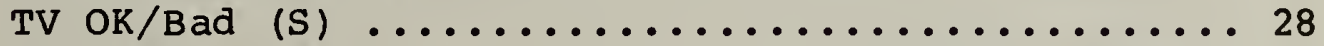

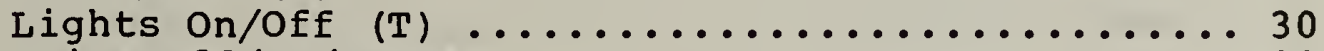

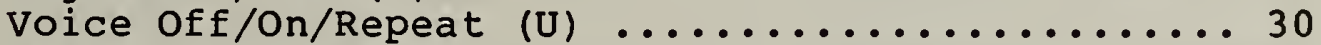

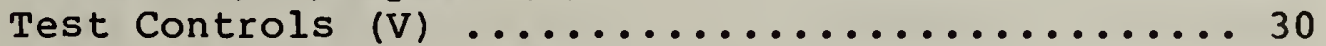

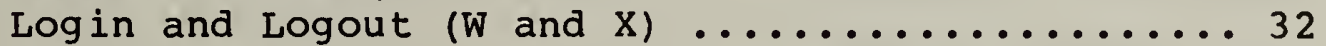

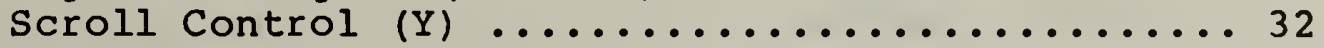

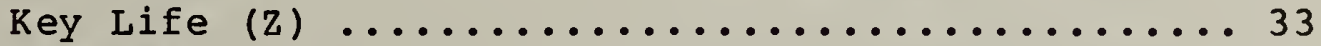

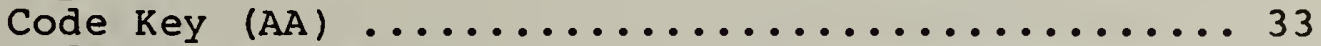

Code Master Key $(B B) \ldots \ldots \ldots \ldots \ldots \ldots \ldots . \ldots \ldots$

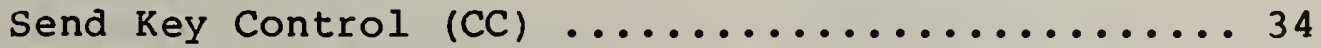

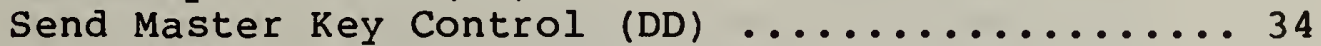

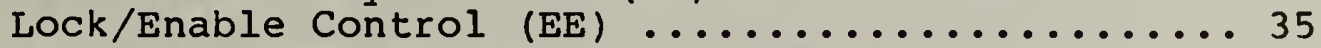

Call BAF (GG) and Call ARF \& BAF (FF) ......... 35

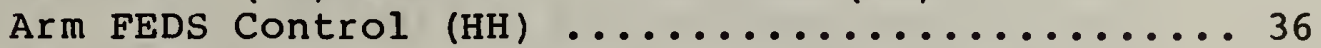

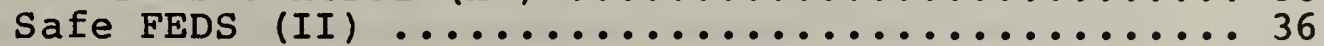

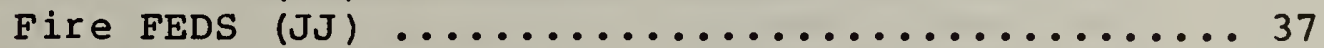

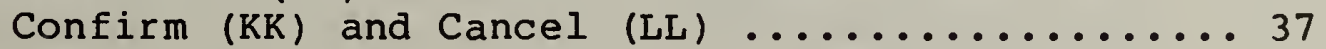




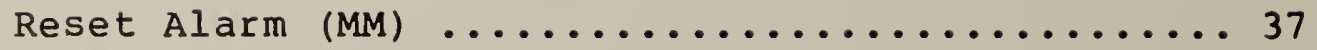

Date Display $(\mathrm{NN}) \ldots \ldots \ldots \ldots \ldots \ldots \ldots \ldots \ldots . \ldots \ldots$

Time Display $(\mathrm{OO}) \ldots \ldots \ldots \ldots \ldots \ldots \ldots \ldots \ldots . \ldots \ldots$

GCS HARDWARE DESCRIPTION ...................... 38

Console Processor Interface Boards ............. 39

GCS Special Interface Boards ................. 39

Switch Debounce .........................40

Lamp Drivers ............................ 40

LCD Decoder/Drivers ...................... 41

Keypad/10-digit Display .................. 41

Monitor Pointing Switch .................. 42

CCTV subsystem .......................43

Cameras .............................443

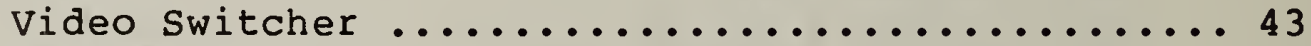

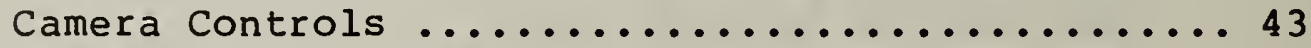

Camera Motor Drive ....................... 44

Voice Unit ............................44

Sonic Alarms ......................... 45

Console shell .......................... 45

Cabling $\ldots \ldots \ldots \ldots \ldots \ldots \ldots \ldots \ldots \ldots \ldots \ldots \ldots \ldots \ldots \ldots \ldots \ldots$

Power supplies .........................46

SIMULATION SOFTWARE $\ldots \ldots \ldots \ldots \ldots \ldots \ldots \ldots \ldots \ldots \ldots \ldots \ldots \ldots$

Software Design ....................... 47

The Console Process ......................48

The scheduler .......................... 48

The Device Driver ....................... 48

The Clock .............................. 50

The Message Handler ....................... 50

The Message Handler and the DDU Manager ........ 53

The DDU Updater ........................ 53

The Console Monitor .................... 53

The Light Monitor ...................... 53

The scroller ............................ 54

The Debugger .......................... 54 
The Event Process $\ldots \ldots \ldots \ldots \ldots \ldots \ldots \ldots \ldots \ldots \ldots$

Console Handler ..................... 56

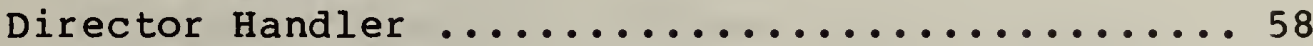

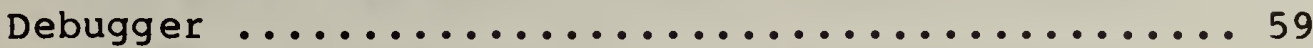

The Director Process ...................6 60

EXPERIMENTS USING THE SYSTEM $\ldots \ldots \ldots \ldots \ldots \ldots \ldots \ldots$

Validation of system Performance ............61

Preparation of Training Materials .......... 62

Tests of Training Materials ..............64

Test by an Electronics Technician ..........6 64

Test by a secretary ....................6 64

Training of Military Personnel $\ldots \ldots \ldots \ldots \ldots \ldots 6$

SUMMARY AND CONCLUSIONS $\ldots \ldots \ldots \ldots \ldots \ldots \ldots \ldots \ldots \ldots$

Console Design Changes .................6 67

10-Digit Keypad ......................6 67

Selected Zone Status Display ..............66 68

Organization of Status Display on DDU ........6 69

Log in/Logout ........................ 70

CCTV Facilities .................... 70

Pan/Tilt/Zoom Control .................... 71

Monitor Pointing Switch ................. 71

Scroll Control ....................... 71

Emergency Controls .................... 72

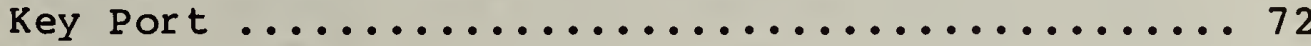

Electronic Key ........................ 72

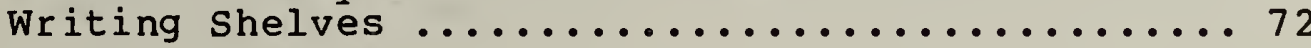

Summary of Changes $\ldots \ldots \ldots \ldots \ldots \ldots \ldots \ldots \ldots \ldots \ldots$

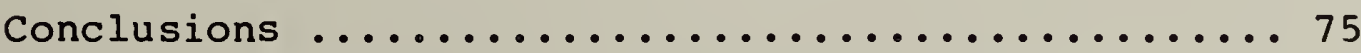

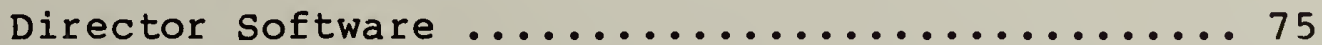

GCS Video .......................... 76

Features Not Implemented $\ldots \ldots \ldots \ldots \ldots \ldots \ldots 76$

BIBLIOGRAPHY AND REFERENCES $\ldots \ldots \ldots \ldots \ldots \ldots \ldots \ldots \ldots$

GLOSSARY OF ACRONYMS AND ABBREVIATIONS $\ldots \ldots \ldots \ldots \ldots 78$

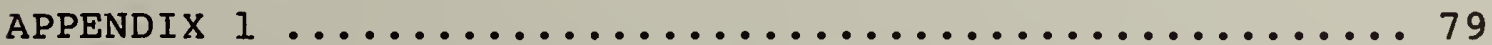


Outline of GCS Console Instruction Course ....... 79

A Sample Scenario .................... 85

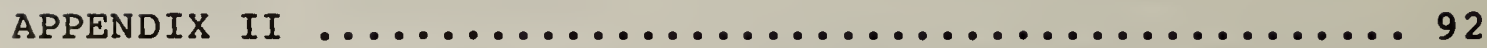

Summary of Responses to Request for Comments ..... 92 


\section{LIST OF FIGURES}

Figure 1A. Block Diagram of CSSMRS . . . . . . . . . . . 2

Figure 1B. Expanded Block Diagram of CSSMRS . . . . . . . . 3

Figure 2 . Guard Control Station . • . • . . . . . . 10

Figure 3. Overview of the GCS Console . . . . . . . . . 12

Figure 4. Digital Display Unit . . . . . . . . . . . . . 13

Figure 5. Alarm Message . . . . . . . . . . . . . 14

Figure 6. More Alarms Message . . • . . . . . . . 14

Figure 7. Zone Specific Alarm Information . . . . . . . 15

Figure 8. A Guidance Message at the Bottom of the Display . . . 16

Figure 9. Right-hand Monitor Pair . • . . . . . . . 18

Figure 10 . Left-hand Monitor Pair . . . . . . . . . 19

Figure 11 . Site Status Summary Display . • . • • . . . 20

Figure 12 . Horizontal Console Panel . . . . . . . . . . 22

Figure 13 . Center Group of Controls . • . . . • . . . . 23

Figure 14. Right-hand Control Group . • . . . . . • . 26

Figure 15 . Left-hand Control Group . . . . . . . . . 29

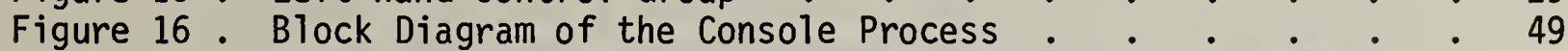

Figure 17. Organizational Block Diagram of Event Process Software : 55

Figure 18. Arrangement of CCTV Cameras and Still Pictures . . . 63

Figure 19. Console Back Panel . • . • . . . . . . . 73

Figure 20 . Console Table Top $\quad$.

\section{LIST OF TABLES}

Table 1. Site Status Summary Display . . . . . . . . 21

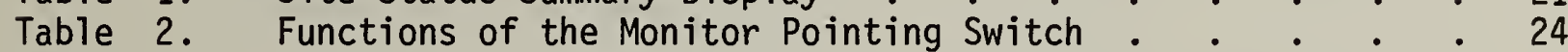

Table 3. Test Control Functions . . . . . . . . . . 31

Table 4. List of Messages from Console Process to Event Process. . 51

Table 5. List of Messages from Event Process to Console Process $\quad 52$

Table 6. List of Messages from Director Process to Event Process . 57

Table 7. List of Messages from Event Process to Director Process . 58

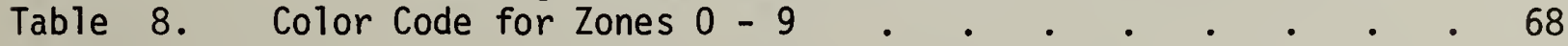



Simulation of the Guard Control station

in a Computerized site security

Monitor and Response system

R. T. Moore

A. W. Holt

A. L. Koenig

A. Mink

G. Nacht

This report describes a mock-up of a Guard Control station that was used in simulating the performance of this component of a Computerized Site security Monitor and Response system. The mock-up was interconnected to three microcomputer systems in an arrangement that permitted simulation of physical security scenarios in an interactive mode. Seven different individuals were allowed to act as operators of the Guard Control station Console following a brief period of training. Based both on observations of their actions and on their comments, it was determined that the CSSMRS concepts were effective and easy to learn. The Console was judged to have met most design objectives, but certain controls and displays were identified as candidates for minor modification or relocation to further improve the overall effectiveness of the console.

Key words: alarm annunciation; physical security; security systems; simulation.

\section{INTRODUCTION}

The Computerized Site Security Monitor and Response System (CSSMRS) [1] was conceived as an integrated, computer based system that could enhance the physical security of storage sites for nuclear weapons and materials through the use of state-of-the-art technology. It proposed a distributed network of microprocessors, each associated with the security measures allocated to a protected zone. These measures might include sensors to detect an intrusion, physical or psychological deterrents to forced entry, closed circuit television to assess sensor alarms, and features to aid in the control of access to that zone. 
Each of these remote microprocessors communicates with a central, triply redundant, computer system via a dual loop, fiber optic, CROSSFIRE[*] data communications network. The central computer complex maintains a continuously updated record of the status of each of the security zones. It presents appropriate information and instructions to each of the two Guard Control Stations, (GCS) as well as to higher command levels. It receives, relays, or executes commands from the GCS. A highly simplified block diagram of CSSMRS is shown in figure $1 \mathrm{~A}$.

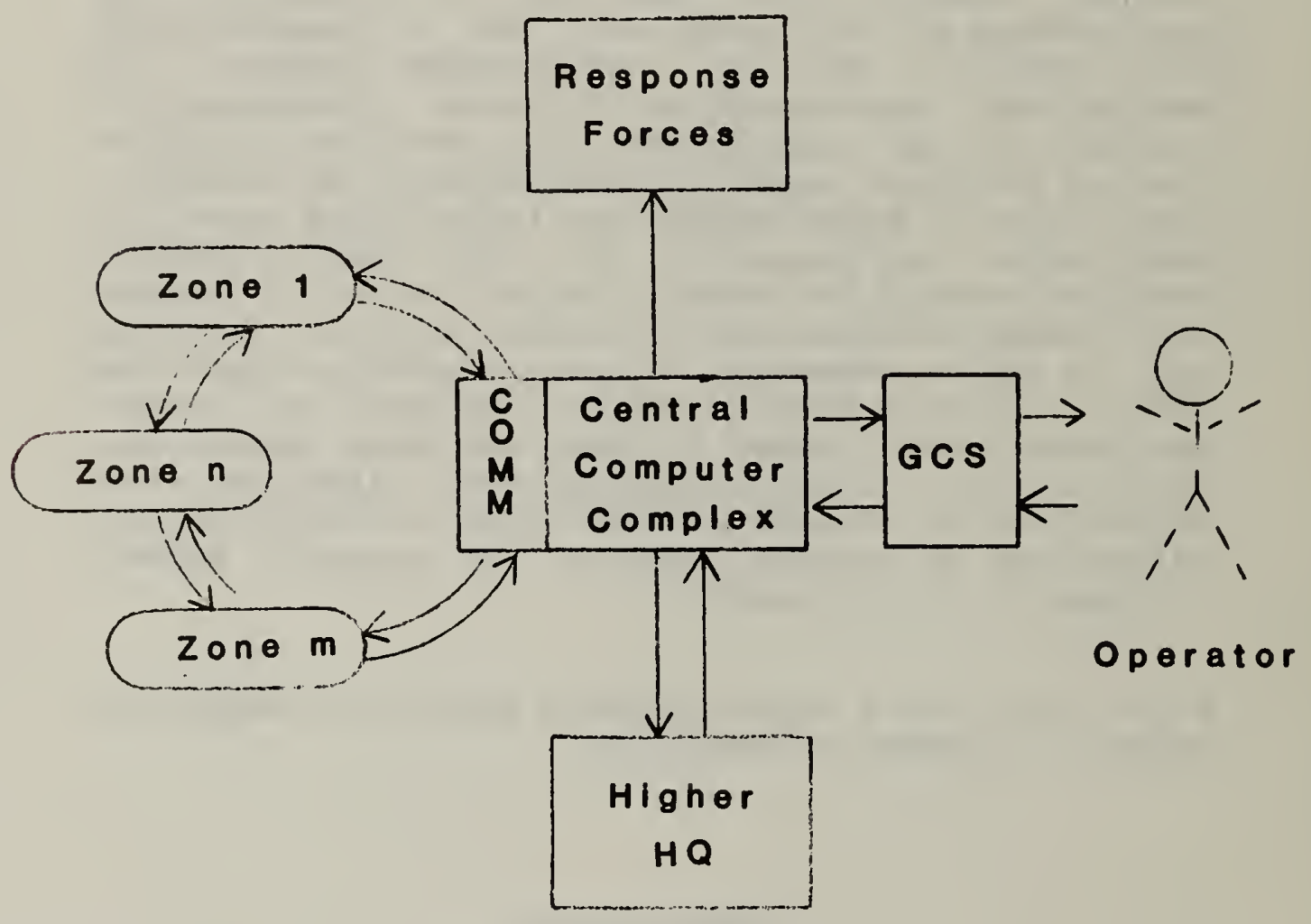

Figure 1A. Block Diagram of CSSMRS.

In figure $1 B$, the block diagram of the CSSMRS has been expanded somewhat to show additional functional details.

[*] The CROSSFIRE data link is an invention of Arthur W. Holt. 


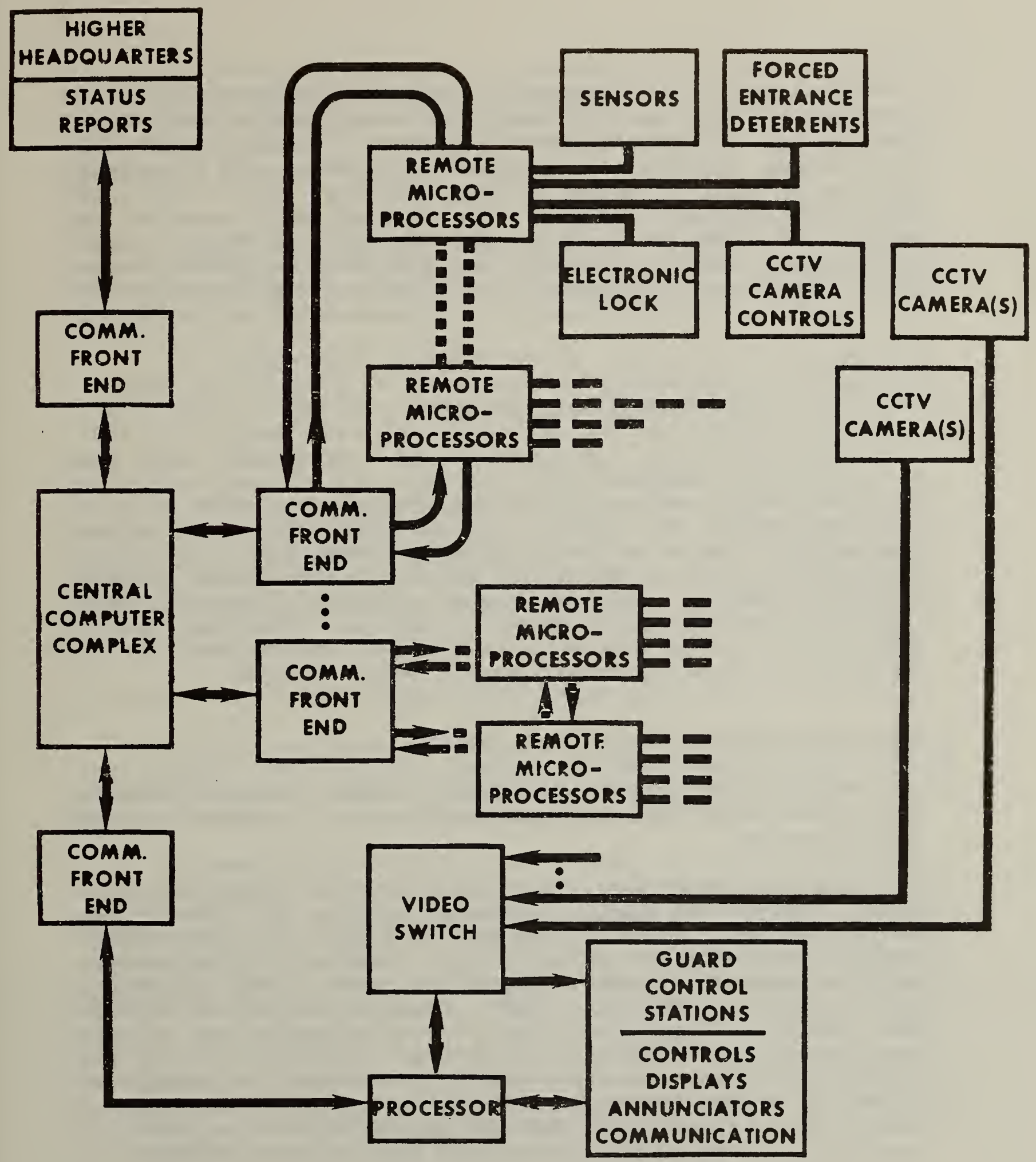

Figure 1B. Expanded Block Diagram of CSSMRS. 
The CSSMRS was designed to provide timely, accurate and unambiguous information about the site security status and the progress of any attempted intrusion. To the extent that is feasible, appropriate response initiatives are programmed into the system. Changes in site security status and the resulting response actions are automatically reported upchannel to higher command levels. Backup and reserve forces are automatically called out in the event of certain identifiable threat situations, particularly those in which the continued survival of local guard forces might be in doubt.

\section{Design Objectives}

While the overall design objective of CSSMRS was that of providing improved physical security, it was recognized that this objective could only be realized with a robust, reliable system, that is easy to use, and is tolerant of human foibles. Users of an unreliable system will not develop confidence in it. Even when an unreliable system is functioning correctly, there may be an element of residual doubt about the correctness of its operation. A system that is difficult to learn to use or to use, will cause the user to perform more slowly, commit more errors, or both.

\section{Features Contributing to Reliability}

Some of the more significant CSSMRS design features that contribute to system reliability and integrity include the following:

The CROSSFIRE data link connecting the remote microprocessors with the central computer complex (CU) uses a dual loop fiber optic channel that does not produce compromising emanations and is immune to electromagnetic interference. The arrangement is highly tamper resistant, and communication cannot be lost as a result of any single break, even of both fibers as described in [1]. Signalling is at a rate that permits the use of inexpensive, lightly stressed, components but still permits response times that are short compared to human reaction times. The use of dual loops together with a continuous polling mode of operation makes it possible to immediately detect and localize any outage.

Communications between the site and higher headquarters are conducted over two different, alternately routed, hard wire, circuits. Continuous testing insures the prompt detection of any fault in either of these redundant circuits. 
The central computer complex is triply redundant. Correct system operation continues as long as any two of these three computers are operational. A fourth computer is on ready stand-by for plug-in replacement if any one of the three working units should fail.

The use of mechanically rotating memory components has been banned throughout the system. This single design feature eliminates a major, if not the major, cause of computer system failures.

Two independent Guard Control Stations are provided. Either one of these is capable of performing all necessary $\mathrm{man} / \mathrm{mach} i n e$ interface functions. This provides an opportunity for load sharing as weil as providing reliability through redundancy.

Alternative back-up power sources have been specified for all system components. There is a back-up for the back-up at the central station, and the weapon storage magazines are equipped with an emergency, manual override for the electronic lock[*] that does not require electrical power to operate.

Features Contributing to Ease of Operation

The general arrangement of the GCS console is based on the human engineering design criteria set forth in MILSTD-1472A [2]. Other features that contribute to the ease of learning to operate the system include the following:

Minimal special skills are required of an operator. The GCS operator does not have to know how to type in order to operate the system. All controls are functional, and labeled in simple, easy to understand terms. The selection of primary controls that are candidates for actuation under any given circumstance is guided by illuminating those controls. Flashing lights indicate "must choose" options. Steady lights indicate reasonable but non-mandatory choices. Guidance messages are provided in the event an inappropriate control is actuated. Certain critical and potentially consequential actions require that the action be confirmed before it is executed. This provides protection against accidentally invoking one of these actions.

[*] The electronic lock is an invention of Jacob Rabinow and Arthur $W$. Holt. 
Closed circuit television (CCTV) coverage of a zone is automatically selected and presented to the GCS operator whenever an alarm occurs in that zone. This permits immediate assessment prior to any action on the part of the operator. This is particularly useful in protecting against high speed penetration of an outer perimeter zone where the penetrator is within the field of view of the camera for only two or three seconds.

Information is presented to the operator both visually and audibly. Alarms are accompanied by a series of sonic alerts, and acknowledgement must be made in less than 40 seconds or reserve forces (BAF) are called out automatically. Alarm information is displayed on a Digital Display Unit (DDU), and is also articulated by a speech synthesis system. The last audible message may be repeated by the operator on command.

Special steps have been taken to protect the operator from information overload. When there are multiple alarms, the details about only one of them are presented to the operator. The display does indicate the number of other alarms so that the operator is aware of the backlog without being overwhelmed by its detail. Alarms, other than the one currently being displayed, are held in a rotary queue. They can be brought up sequentially by actuating a single control. The operator does not have to know the identity of any alarmed zones to call them up on the display.

Site status information is displayed whenever any zone is selected that is not in an alarm condition. In addition, overall site summary information is displayed by a cluster of colored indicator lamps for quick look assessment by supervisory personnel.

Controls for the Pan, Tilt and Zoom functions of CCTV cameras that are so equipped have been human engineered so that the hand motions are the same as required if the operator were holding the camera in his hand.

\section{Purpose of the Mock-up}

The Guard Control station mock-up (GCS) was assembled to provide a test bed that could be used with computer simulation to test and evaluate the various novel design features affecting the man-machine interface in the CSSMRS. In particular, it was intended to provide information about the ease or difficulty that non-technical personnel would have in learning to use the system, and to show whether or 
not that use would be direct, easy, and relatively error free. It was expected to help provide answers to questions of the following types:

Do users find that the Guard Control station is friendly or intimidating? Hopefully, the increasing availability and popularity of video games may have provided opportunities for many non-technical individuals to use computer based devices and learn to feel comfortable with them.

Are the locations of the controls and indicators handy and easy to use and do they have clear and easily understood labels and nomenclature? This was a major objective in the layout of the GCS, but only actual operating experience would reveal how well that objective has been attained.

Are the cues and guidance messages adequate to support effective operation of the GCS by an operator with limited technical background and minimal training? Extensive audible and visual cues have been provided including sonic alarms, voiced messages, and the use of colors and lights in the controls and indicators.

Are the displays, including both graphics and video, adequate in terms of size, resolution, location, and information content?

These and related questions could best be answered by the construction of a GCS that could be used in a simulation environment to provide a highly realistic demonstration of the behavior of such a station in a real CSSMRS. Such a model would provide nearly all. of the audible, visual and tactile stimuli to its operator that would be provided by a real GCS. It would react to $h$ is responses in the same way as a real GCS. By teaching operators to use the GCS and by observing their actions during the conduct of threat scenarios on the simulator, it was expected that insight could be gained regarding the adequacy of the GCS design and its interaction with the balance of a cSSMRS. 


\section{INITIAL DESIGN}

A detailed set of performance specifications was developed from a general description of the GCS component of CSSMRS [1]. These were in the form of state descriptions and contained a list of all GCS controls, indicators, and displays, and reflected their response to differing stimuli.

A complete state space description for the GCS was found to be too large to be accomplished within available resources. Instead, a number of major conditions were identified, which could co-exist at the same time. A state space description of each of these major conditions was specified which assumed the major conditions existed independent of each other. During a second iteration of these independent state spaces, an attempt was made to include variations resulting from the co-existence of other major conditions. The resulting specifications provide a description for each state of each major condition. This description consists of the current condition of the GCS (its initial state), the action causing a transition and the resulting condition of the GCS (its next state). The condition of the GCS, as described includes the controls that are active, the illumination state of each indicator and control, the contents of each CCTV monitor, any messages on the DDU that are of concern to the current state, and the contents of a new message and its disposition for the DDU, the Voice Unit and the Logging Printer.

Specifications for the physical layout of the Console portion of a GCS were taken from the MIL-STD-1472A [2]. A cardboard mock-up was made so that experimentation with various positions and combinations of the controls, indicators and displays could be conducted.

At this point it was necessary to consider the fabrication of the GCS Console, the hardware to be used, and the software required to drive the hardware. For the sake of expediency and economy, the selection of hardware and software were based on availability and familiarity rather than optimization of function and speed.

The hardware would be centered around existing, available processors. The GCS Console, its lights, switches, displays, and the interfaces between them and the processors would be obtained commercially, if they were available. Otherwise they would be custom designed and fabricated. 
The software would be written in the $C$ language [*] with some assembly lanquage where necessary. Three cooperating processes were involved in the software design. One, the Console Process, was to service the console inputs and outputs. The second, the Event process, was to service and maintain the history of events from the individual zones via the C.U. and from the Console Process. A third process, the Director Process, was also required to coordinate events and simulate the C.U. and the actions that could occur at the various zones.

Each process was placed on a separate processor. This allowed the Console process to run stand alone in a polling mode, while the other processes were run under UNIX [3] [4] an interupt driven operating system. Running under an operating system was possible only for this mock-up, since time was not as critical for the Event and Director processes as it was for the Console Process. UNIX also served as the development system environment.

These initial design decisions led to an efficient implementation of the GCS simulation model in a straightforward manner.

SYSTEM CONFIGURATION

The principal GCS components of CSSMRS in this simulation model are shown in figure 2. They consist primarily of three parts, the Console itself, the Console Process and the Event Process. GCS features detailed in NBSIR 79-1725 that were not implemented in this model include the Logging Printer, the Audio Recorder, the Geographics Display Unit, the Radio, Telephone and Intercommunications facilities, and the Duress alarm functions.

The Console is the principal interface between the Operator and CSSMRS. It provides the Operator with closed circuit television (CCTV) pictures of the site, interactive controls, and both audio and video messages. The messages can be of three types; routine status messages, alarm messages, or operator guidance messages. Status messages may describe the state of equipment or conditions on the site,

[*] UNIX is a trademark of Bell Laboratories. 


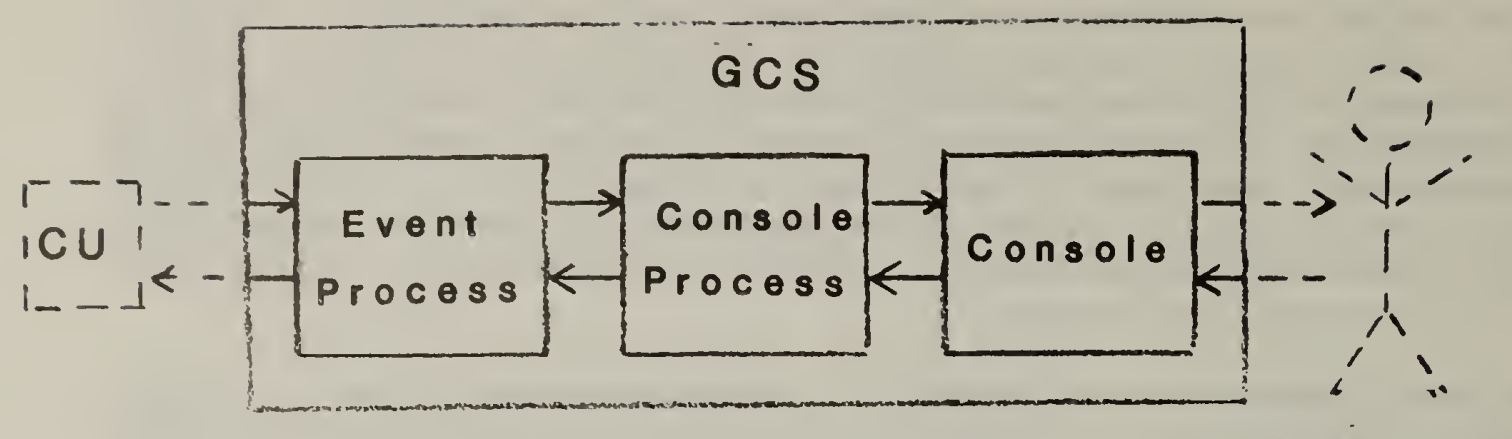

Operator

Figure 2. Guard Control station

such as malfunctioning sensors, presence of uncleared alarm conditions, or zones in access condition. A new alarm condition replaces status messages and demands positive acknowledgement by the operator. When that acknowledgement occurs, the message is expanded to provide details about the nature of the alarm and suggested Operator actions. Operator guidance messages are presented to indicate results of tests, candidate Operator actions, or actuation of an inappropriate control. As a message is presented to the operator on the Digital Display Unit (DDU), it is also vocalized by a Voice Unit. Controls on the Console allow the Operator to either have the last voice message repeated if desired, or to turn the Voice Unit off if the audio is distracting.

The function of the Console Process is to service the Console. It polls the state of the Console's controls and detects any changes. It exchanges messages with the Event Process and maintains a necessary subset of the system's database for its use. It checks the legality of the Operator's actions and generates guidance messages for certain incorrect actions. It reflects the current state of the site by various indicator lights on the Console, messages displayed on the DDU, and outputs by the Voice Unit.

The function of the Event Process is the maintenance of the system's database, i. e., the history of events from the Console Process and the CU. It maintains a detailed list of the state of each zone (secure, accessed, alarmed, etc.,) and the state of each Sensor and Forced Entry Deterrent device (FED) at each zone (disabled, active, alarmed, etc..). 
It maintains a list of all DDU messages currently resident at the Console Process. The Event process communicates with the Console process and with the $C U$. In many cases the Event process is able to determine the preferred choice of operator response to the current site status and, via messages to the Console process, issue guidance prompts to the Operator.

A third process, the Director Process, was necessary to simulate actions at the various zones which were forwarded via the dual loop communications network, filtered through the CU, and passed to the GCS. This was the means by which sensor alarms, maintenance functions, equipment failures, and other zone and $C U$ events were simulated. Since this Process is not actually part of the GCS model, it will not be discussed in depth in this report.

Each of the processes was placed on a separate processor. The reasons for this were dictated by requirements and system considerations and will be discussed in later sections of this report. Modified 16-bit parallel ports were used for communications between processes. Since the standard circuit cards for these ports were designed for communication between a processor and a peripheral device, they had to be modified for a processor-to-processor communication link.

Description of the GCS Console

The GCS Simulation Model is shown in figure 3. The Console is mounted on a dual pedestal base where each pedestal is a standard 19 inch equipment rack. The pedestal on the left side contains the Console Processor, interface and driver circuit cards, and power supplies. The pedestal on the right side contains the Voice Unit and video switches for the CCTV's.

The vertical portion of the Console may be considered as four functional sections: the Left and Right CCTV Monitors Sections, the status summary Display and the DDU Section. These will each be described in detail below. The letters in the illustrations are used to identify the various components of the system in the descriptions that follow. 


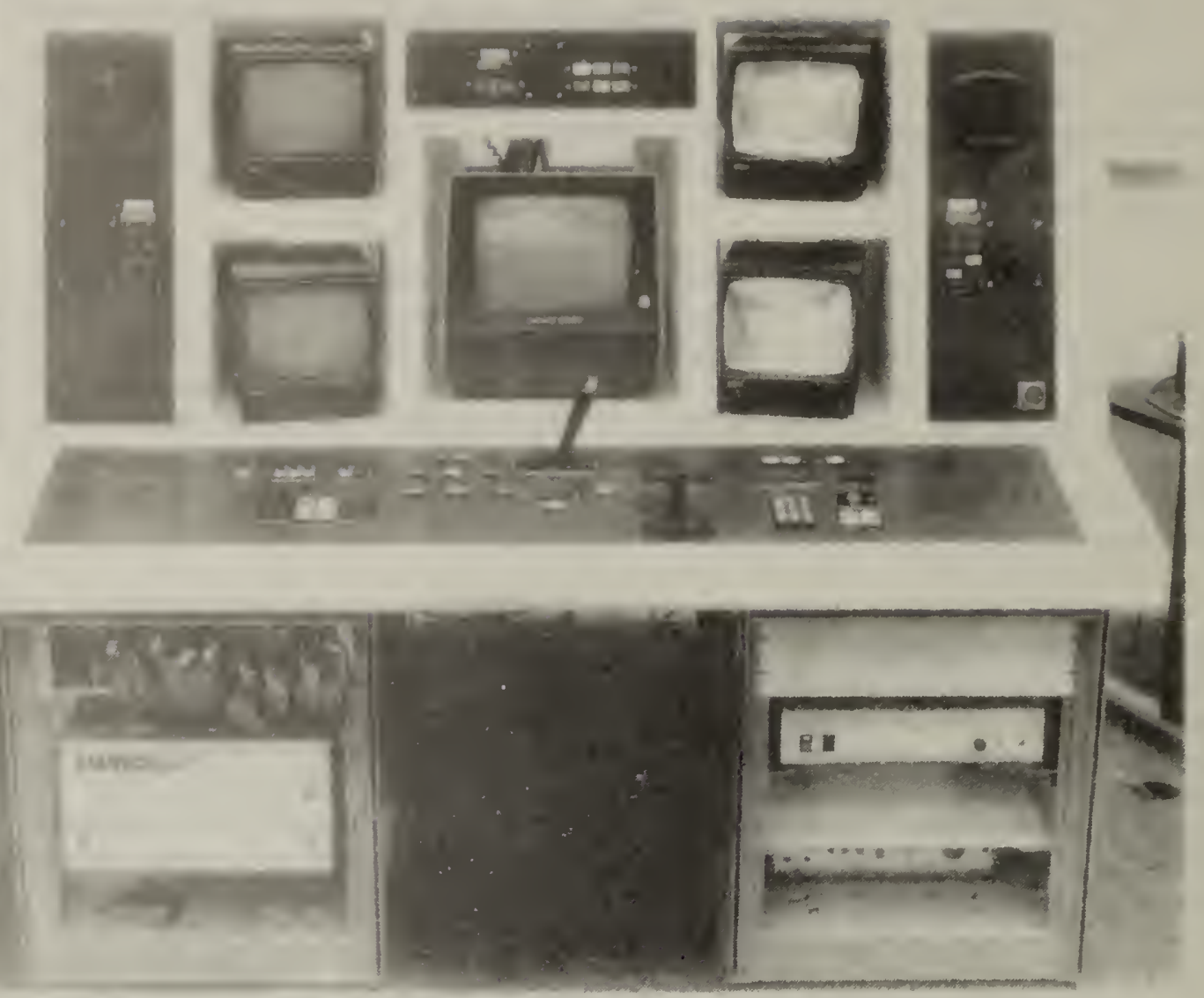

Figure 3. Overview of the GCS Console

Digital Display Unit (A)

The Digital Display Unit, DDU, figure 4, is central to the GCS. It is a $30.5 \mathrm{~cm}(12$ inch) diagonal screen cathode ray tube capable of displaying information using a format of 24 lines of 80 characters each. It is also capable of displaying special information using any combination of double width or double height characters. For this simulation model the DDU displays four classes of information: new alarm information, alarmed zone information, status information and guidance messages. These messages were also audibly annunciated when they were initiated. 


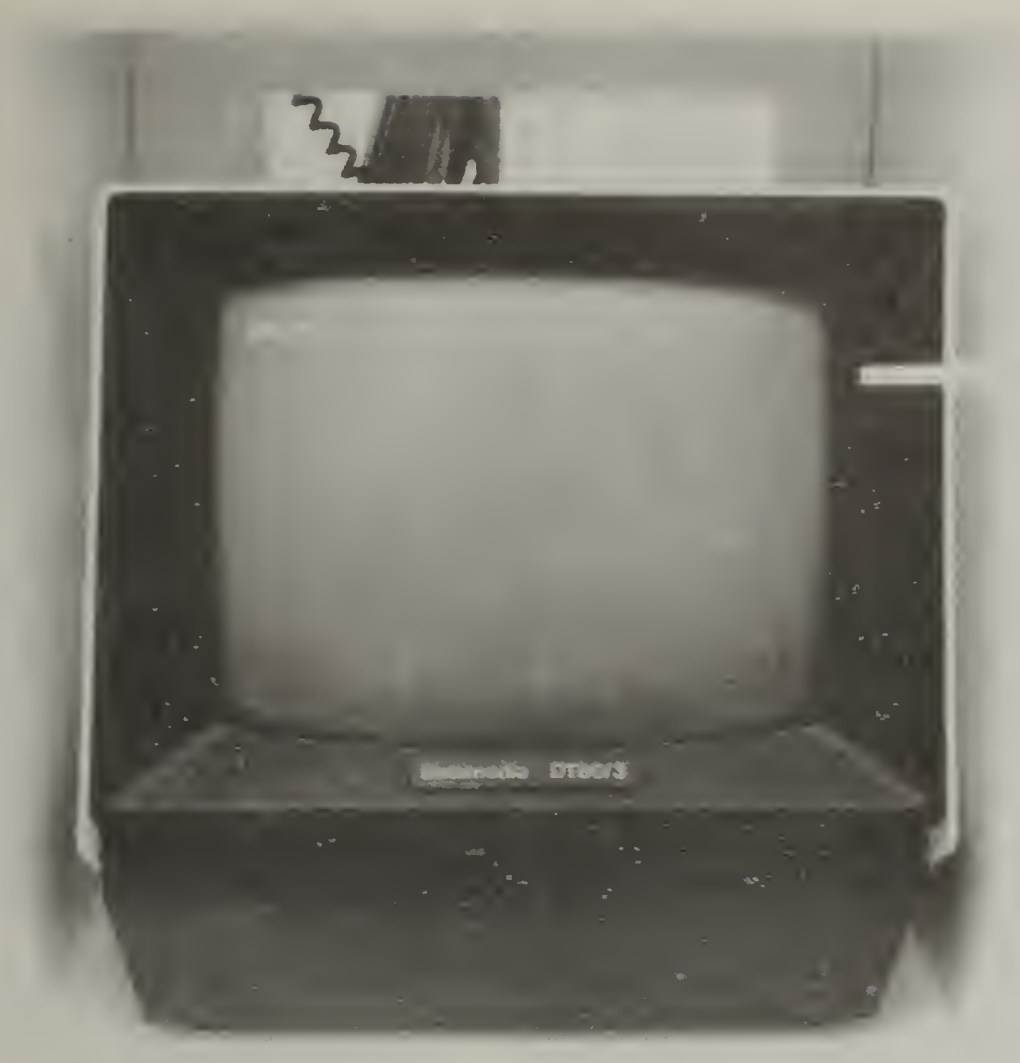

A

\section{Figure 4. Digital Display Unit}

When an alarm occurs (a sensor has been activated), the information currently displayed on the DDU is removed and replaced with a special "Acknowledge" request to the Operator, figure 5. This also starts an audio alarm sequence which will be explained later. Other new alarms cause a "More Alarms" message to be added to the screen, figure 6 .

All new alarm messages are double wide except for the word "Acknowledge", which is double height and width. When the Operator acknowledges the new alarm then, depending on the state of the zone, the DDU will display either site status information or alarmed zone information. 


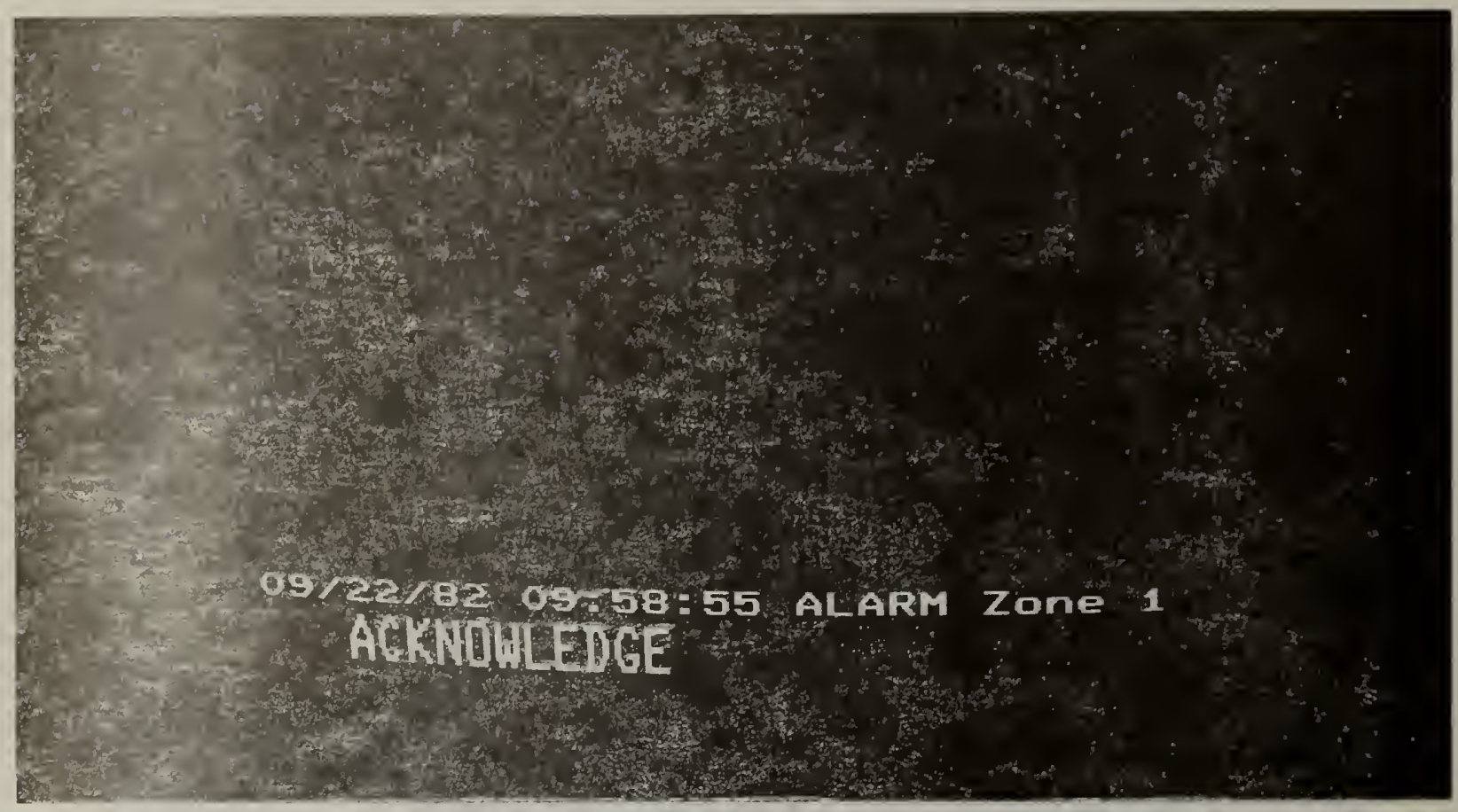

Figure 5. Alarm message.

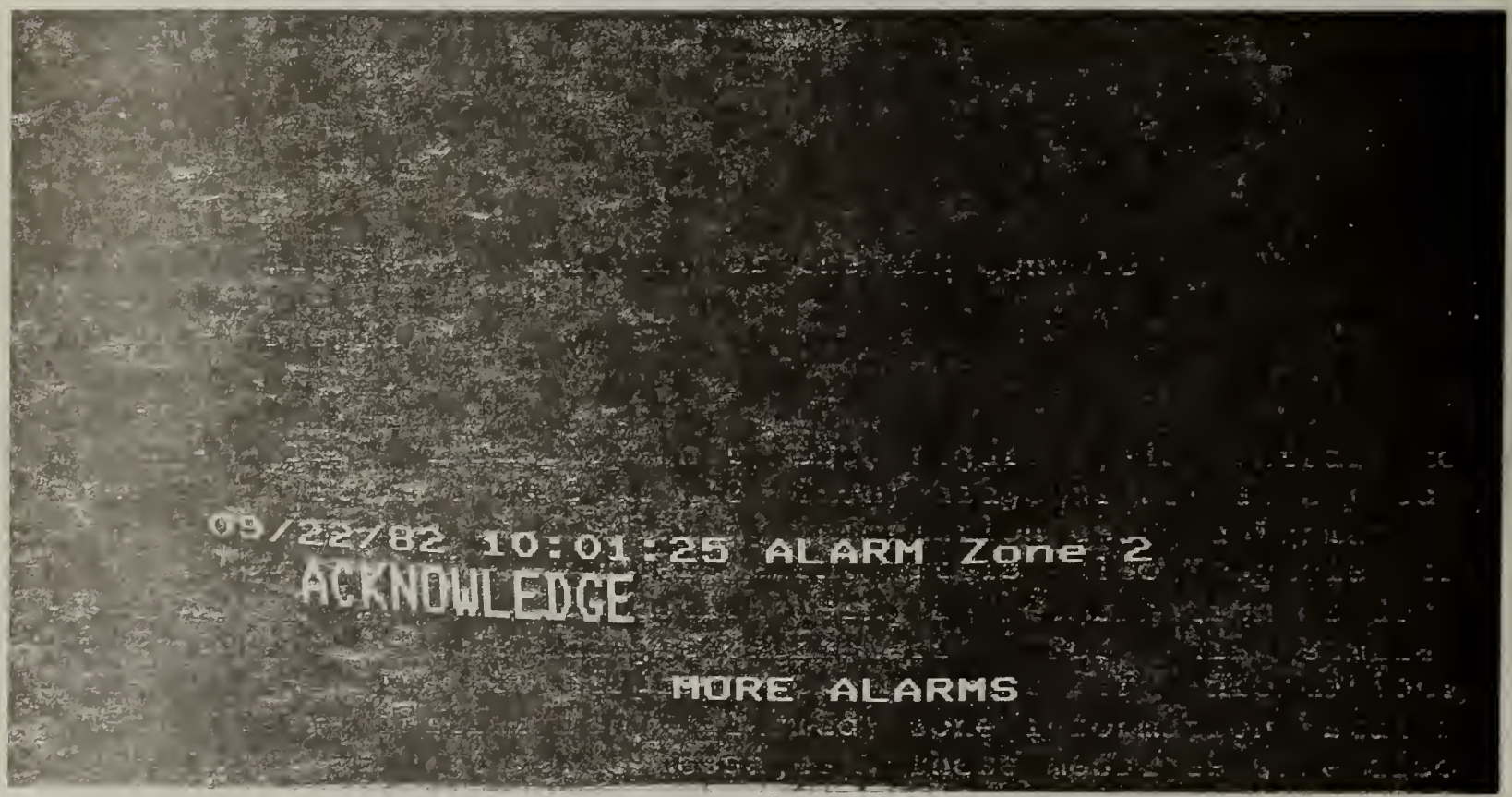

Figure 6. More alarms message 
If the Console controls are associated with or logically attached to an alarmed zone, then the information displayed on the DDU is specific to that zone, figure 7 . It will tell the operator, for example, which sensors at that zone are alarmed, which equipment at that zone is malfunctioning, the state of the Forced Entrance Deterrent systems (FEDS) at that zone and how many other zones are alarmed. All information displayed in this format is double wide.

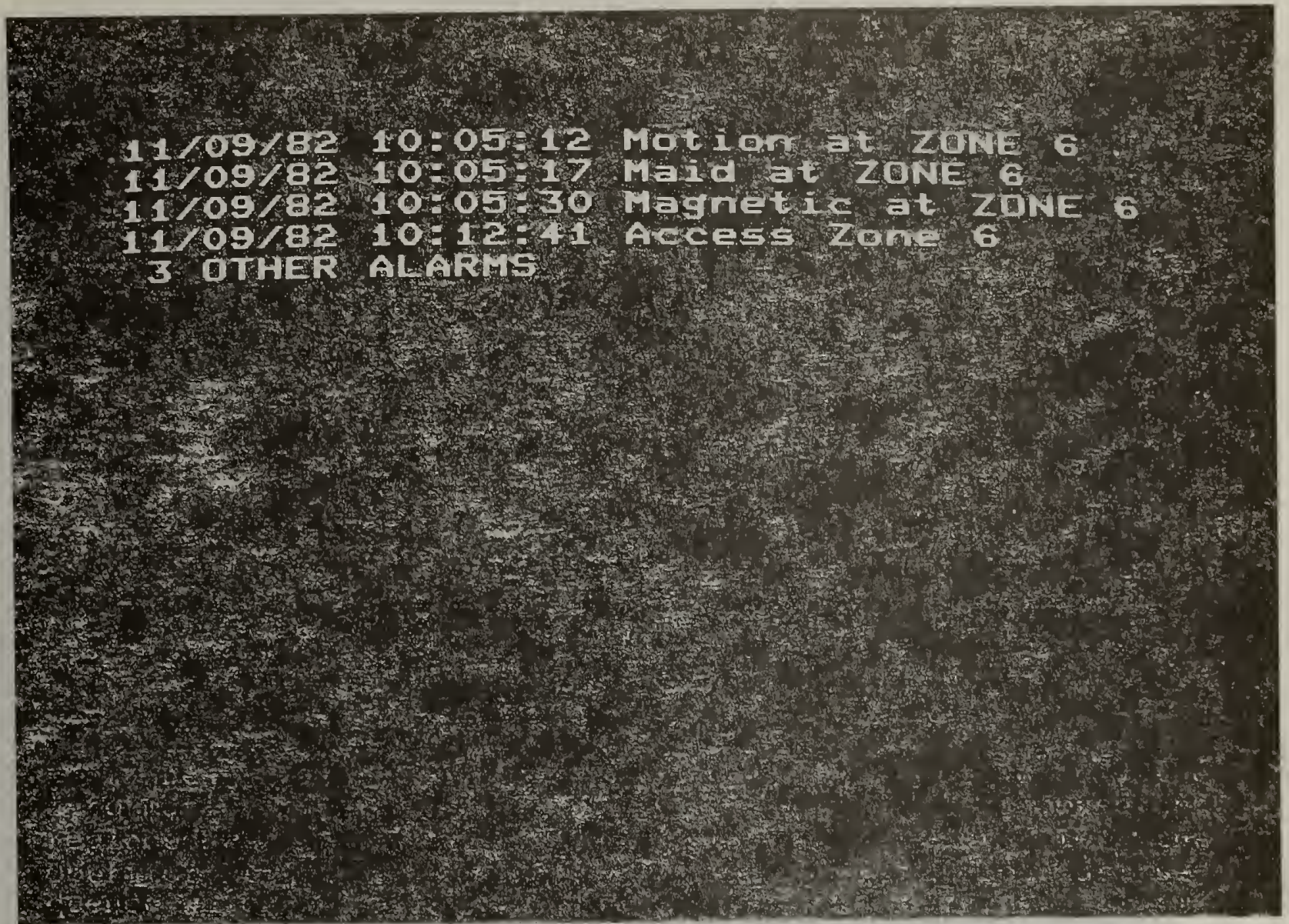

Figure 7. Zone specific alarm information.

If the Console controls are attached to a nonalarmed zone then site status information is displayed, figure 8. This is not zone specific and will tell the operator, for example, which operators are currently logged in, which zones are in access, which equipment is malfunctioning and at which zone, how many zones are in the alarmed state and, if there are any communication 
failures, where and what type of failures have occurred. This information is displayed in the standard sized format except for the number of zones in the alarmed state. This message is double width so that it may be more readily seen.

The bottom line of the DDU is used for guidance messages to the GCS Operator, figure 8. These messages usually indicate inappropriate use of a control or recommended response actions to the current situation.

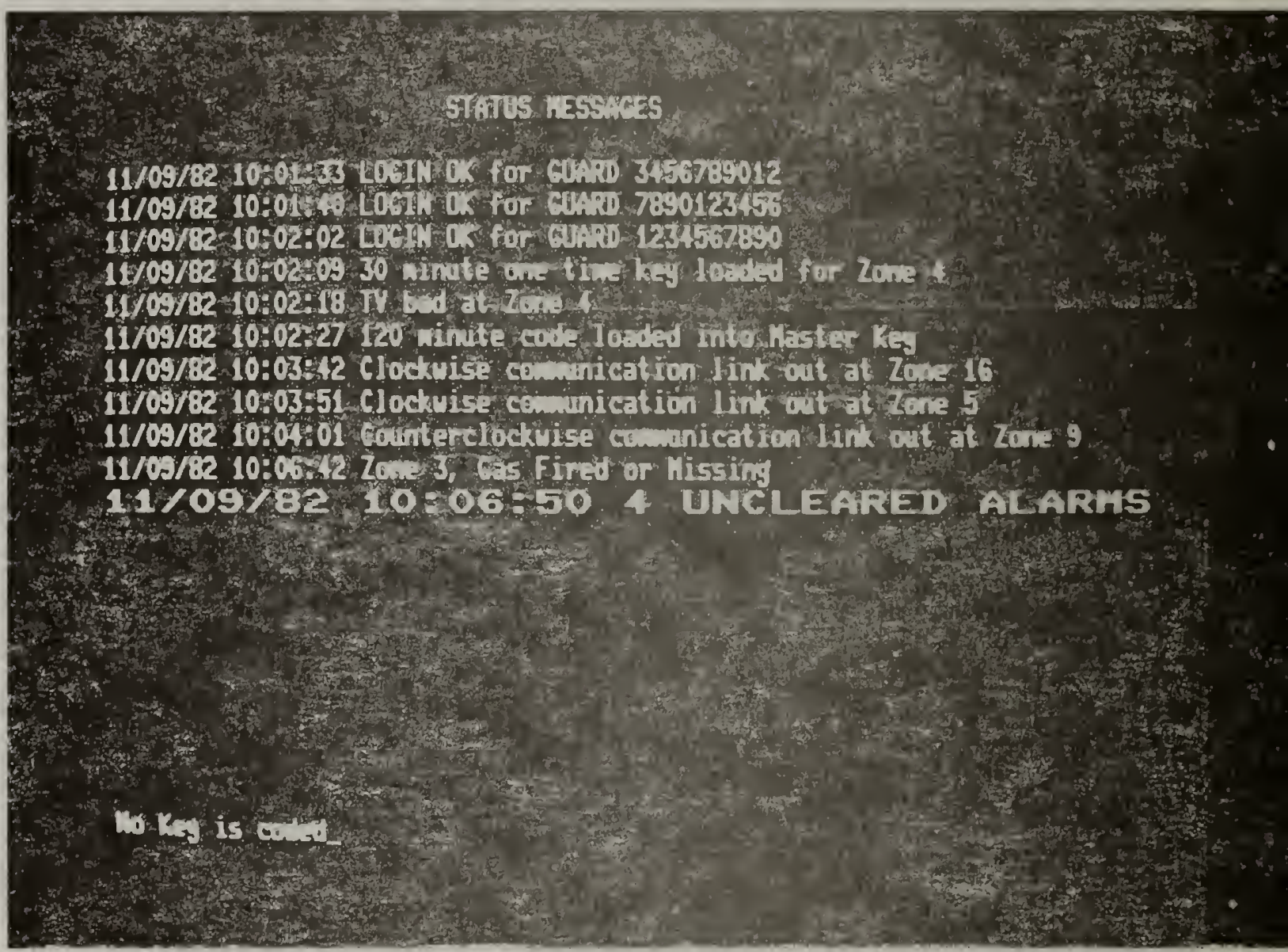

Figure 8. A guidance message at the bottom of the display. 
The detection of a new alarm condition starts a sequence of audio alarms. This sequence is also initiated by the GCS Operator actuating the FIRE FEDS, the CALL BAF or the CALL BAF \& ARF Controls. (These controls are described later in this report.) This audio alarm sequence is terminated as soon as the GCS Operator acknowledges the new alarm condition or verifies the action. The first tone is $2900 \mathrm{~Hz}$ interrupted and lasts for 15 seconds. The second tone is $4500 \mathrm{~Hz}$, steady, and lasts for 15 seconds. The third and final tone in the sequence is a very loud buzzer and lasts until an Operator acknowledges.

CCTV Monitors (C, D)

A pair of CCTV Monitors, figures 9 and 10 , are mounted on either side of the DDU. At the GCS Operators discretion either pair may be connected to the assessment camera(s) at any zone that is equipped with cameras. Normally, the other Monitor pair is dark. When an alarm condition occurs, the previously dark CCTV Monitor pair is automatically selected and connected to the camera(s) at the alarming zone. If there is a pair of cameras at a particular zone then both Monitors are used. If there is only one camera available then only the lower Monitor is used and the upper one is dark. In all cases any Monitor not connected to a camera is dark.

CCTV Monitor Display (E, F)

Associated with each Monitor pair, (see figure 9, for example) there is an LCD numeric display (E) indicating the zone whose camera is connected to the Monitor. Also there is a set of indicators (F) showing which types of camera controls (pan, tilt, etc), if any, are available at that zone. 

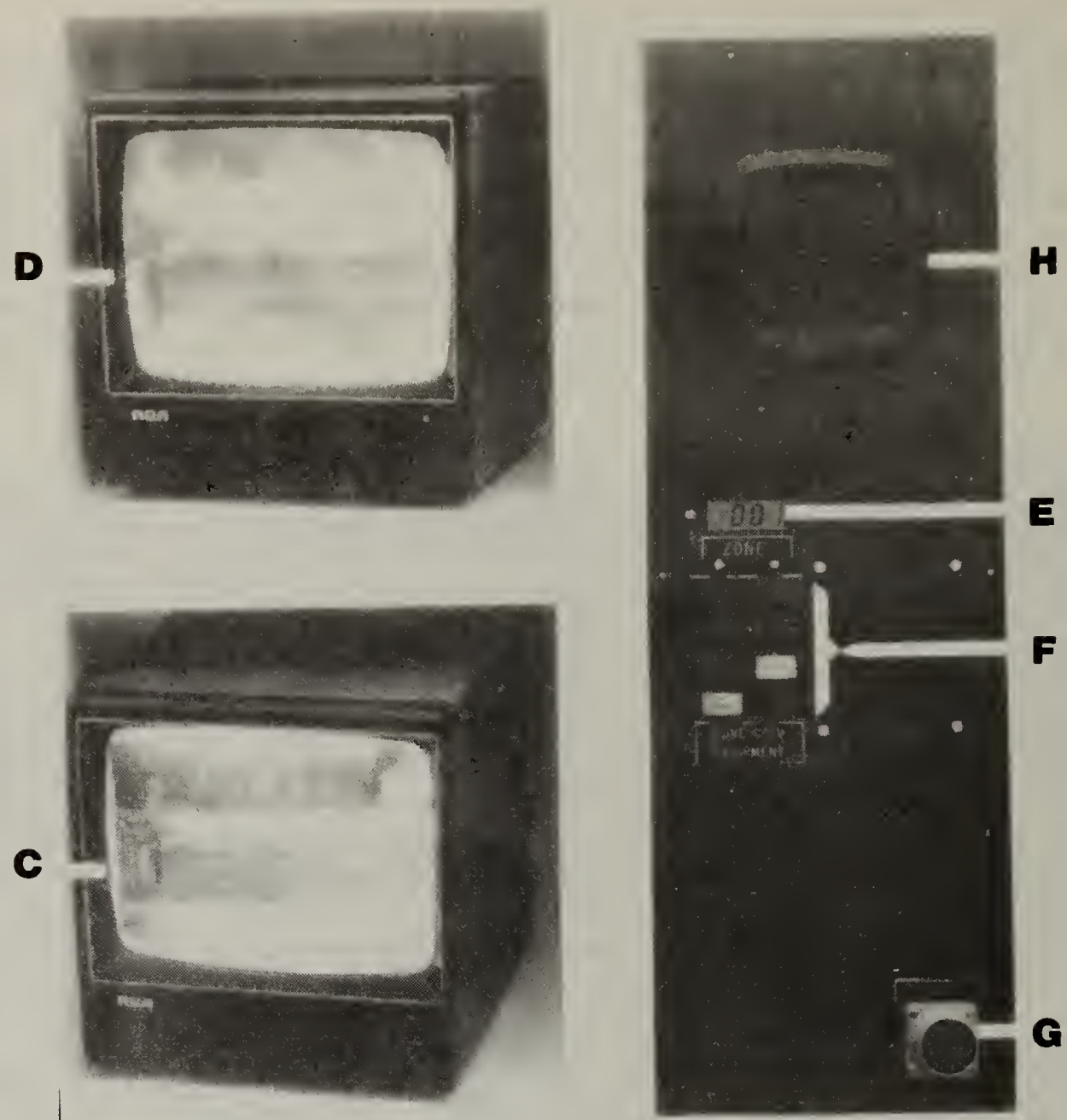

Figure 9. Right-hand monitor pair

Key Port (G)

This is the connector, figure 9, into which the Electronic Keys are inserted. It permits the loading of a random number code and time period for which this code is valid. 

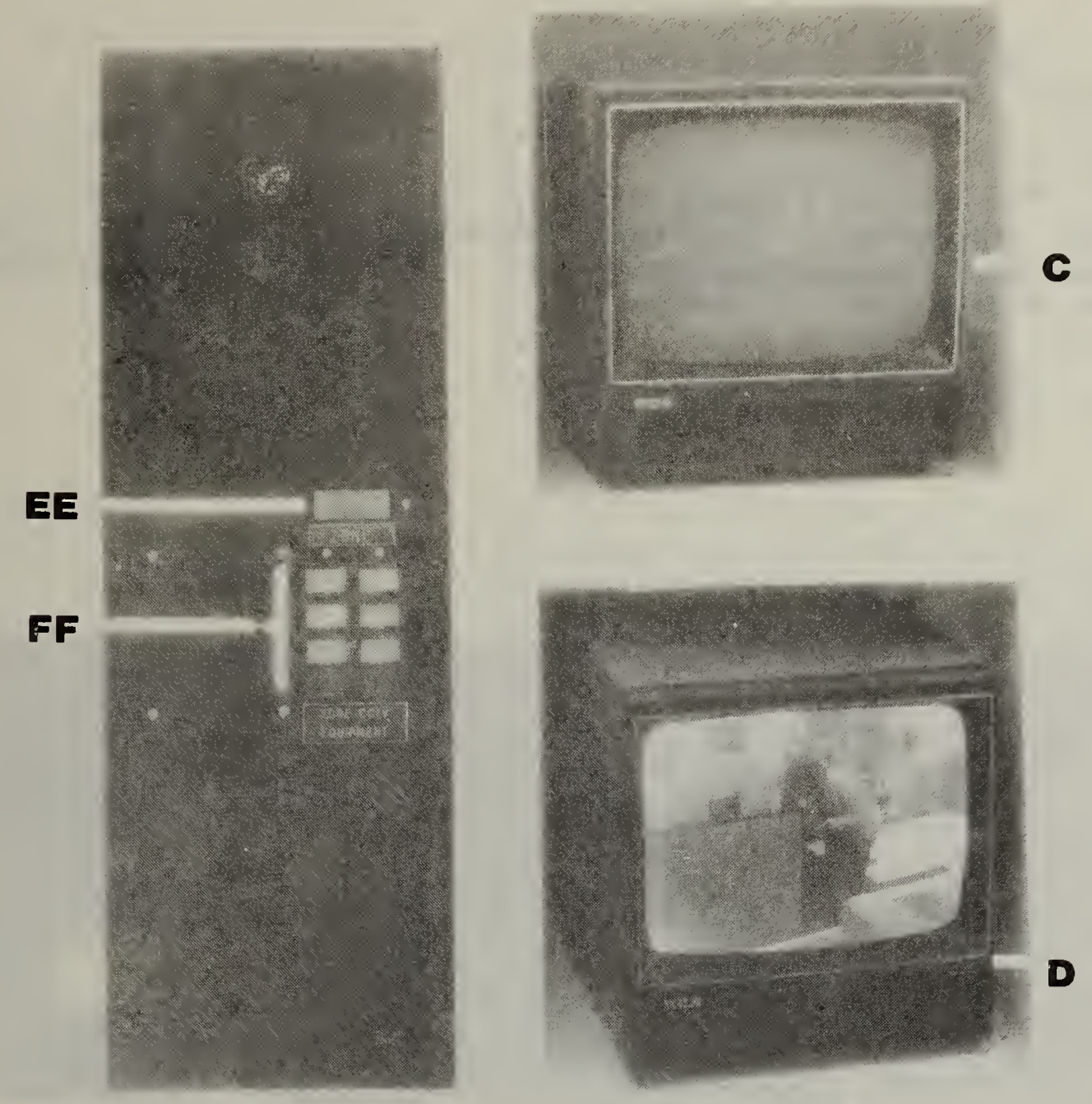

Figure 10. Left-hand monitor pair

Voice Unit Speaker (H)

The GCS Simulation Model is equipped with a speech synthesizer. This permits the annunciation of each message as it is displayed on the DDU, thus providing both audible and visible means of information transfer from the System to the GCS Operator. Figure 9 shows the location of the speaker for this Voice Unit. 
The Status Summary Display, figure 11 , is a group of six indicator lights. These indicators provide site status information to the operator. Their functions are summarized in Table 1.

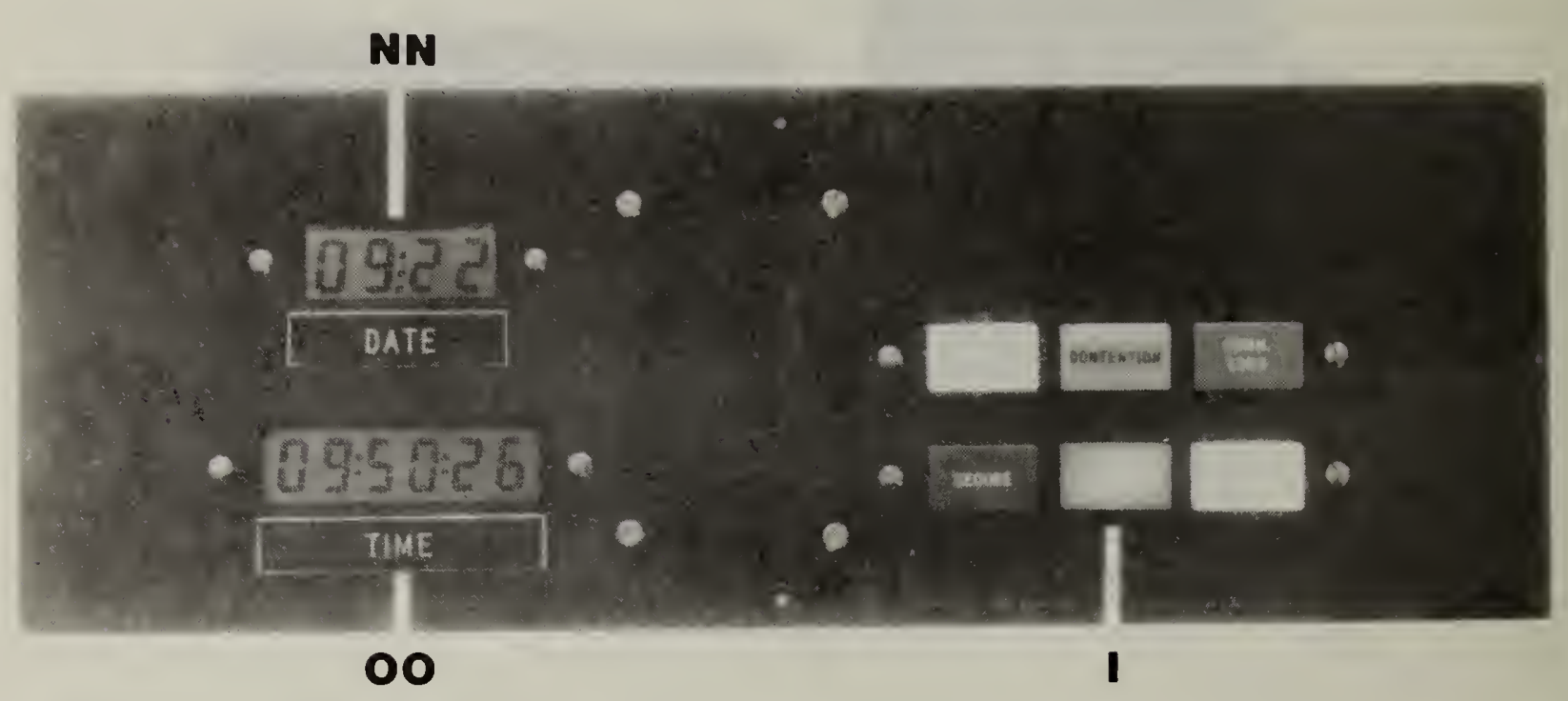

Figure 11. Site status summary display 
TABLE 1 -- Site Status Summary Display

Indicator Color Function

Repairs

Required

Contention

Communication

Loss

Secure

Alarm

Access
White ON when any critical system component is in need of maintenance action, e.g., sensor has failed test or a zone is operating on backup battery power. Can be $\mathrm{ON}$ in company with any other indicator.

Yellow on for the duration of any conflicting or contentious commands issued by the two guard control stations. Can be oN in company with any other indicator.

Blue ON when there is a loss of communication with any zone causing that zone to be assumed to be operating in autonomous mode. Can be on with any other indicator except "Secure".

Green All zones in the site are secure. This light can be ON only with with "Contention" or "Repairs Required". Lighting of any other indicators causes this one to be turned OFF.

Unacknowledged alarm condition exists. This changes to Red (steady) when acknowledgement occurs. Can be oN with any other indicator except "Secure".

Red Acknowledged but uncleared alarm (steady) conditions exist. Can be ON with any other indicator except "Secure".

Yellow Authorized Access is occurring in one or more zones. Can be ON with any other indicator except "Secure". 
The horizontal portion of the GCS console is shown on figure 12. All of the controls that the Operator must handle are located on this horizontal area which is shown in more detail in figures 13 through 15.

\section{is a}

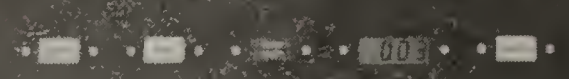

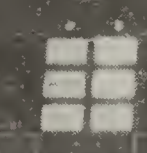

-.

Figure 12. Horizontal console panel

Monitor Pointing Switch (J)

A Monitor Pointing Switch (MPS), figure 13, is provided on the Console to facilitate the rapid and unambiguous switching of CCTV Monitors and attachable Controls from one alarmed zone to another.

The MPS is a two position, toggle switch with a hand sized handle having a light at the outer end. The throw is about 90 degrees. The tip of the handle always points toward the CCTV Monitor associated with the currently attached zone. If the switchable controls are not attached to a zone, then the CCTV Monitor to which the MPS points is dark. The functions of the MPS are summarized in Table 2. 
The indicator light in the end of the MPS handle acts as a prompt to the operator in requesting an acknowledgement when a new alarm condition is detected. It flashes when a new alarm condition is detected on a previously secure site. It flashes alternately with the light in the ACKNOWLEDGE switch (K) if a new alarm is detected at a zone not already alarmed while there are other uncleared, acknowledged alarmed zones present. The light is turned off when the acknowledgment occurs.

\section{JJ FF GG HH II}
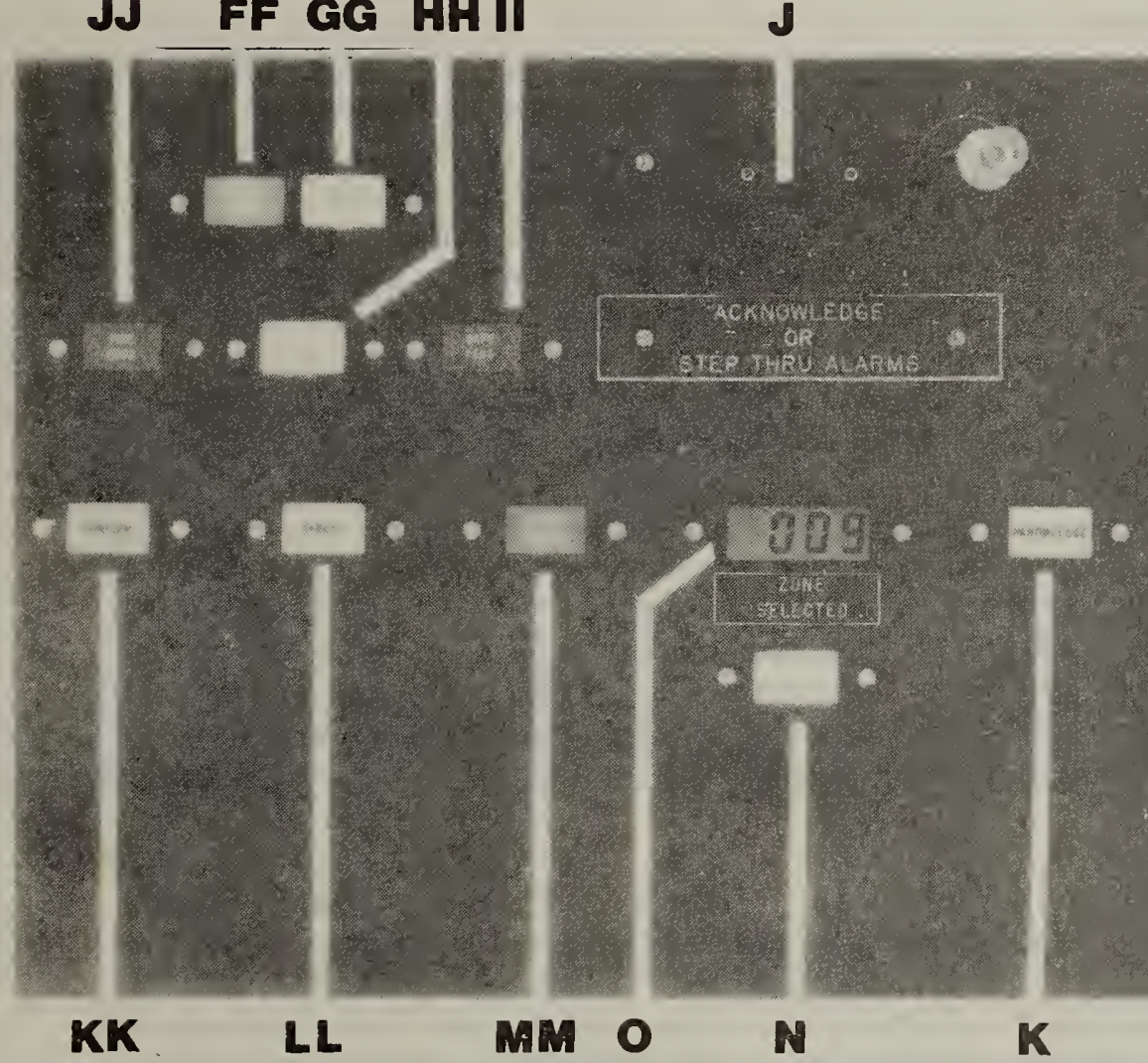

LL

MM

N

$\mathbf{K}$

$\mathbf{P}$

Figure 13. Center group of controls 
TABLE 2 .

Functions of the Monitor Pointing Switch

1) When the MPS is switched from one side to the other, the CCTV Monitors from which it is moved go dark. The dark Monitors are now ready to receive the video from the cameras at any newly alarmed zone.

2) All outstanding, unacknowledged (new) alarms are acknowledged and the zones at which these alarms have occured are added to the alarm queue.

3) If the site is secure (no outstanding, uncleared alarms) and a zone has been selected to which the CCTV Monitors and switchable Controls are attached, then, as the MPS is moved from one side to the other, the video will also move from the Monitors on one side to the other. That is, the video always appears on the side to which the MPS points. The CCTV Monitors to which the MPS does NOT point will normally be dark.

cleared alarmed zone, then, when the MPS is moved from one side to the other, the switchable Controls will be attached to this zone and the video from the cameras at this zone will be displayed on the Monitors to which the MPS now points.

5) If there is more than one acknowledged, uncleared alarmed zone, then operation of the MPS attaches the CCTV Monitors and switchable Controls to each of the alarmed zones in turn. That is, the zone at the top of the alarm queue is attached and the previously attached zone is moved to the bottom of the queue. This way each of the alarmed zones may be rapidly examined in turn merely by moving the MPS from one side to the other.

6) If there are alarmed zones in the queue and a new alarm is detected at a zone not currently attached, then the video from that newly alarmed zone will appear on the previously dark CCTV Monitors (the side to which the 
MPS is NOT pointing). Operation of the MPS will then acknowledge the new alarm and add it to the top of the alarm queue. The MPS now points to the CCTV Monitors where the newly alarmed zone's video is displayed, and the switchable controls are attached to this zone. The CCTV Monitors on the other side will go dark. If the previously attached zone is on the alarm queue, it is moved to the bottom.

Acknowledge (K)

The ACKNOWLEDGE switch, figure 13, is a momentary action, lighted push switch. Its operation will acknowledge all outstanding, unacknowledged alarms, but will not change the zone to which the CCTV Monitors and switchable Controls are attached.

The indicator light in this switch acts as a prompt to the operator to acknowledge newly detected alarm conditions. It flashes if a previously unalarmed sensor is alarmed at a zone already in the alarm queue. It flashes alternately with the light in the MPS handle if a new alarm is detected at a zone not previously alarmed while there are other uncleared, acknowledged alarmed zones present. The light is turned off when the acknowledgment occurs.

Operation of this switch when there are no outstanding alarms to acknowledge causes an audible beep to occur and a guidance message, "No alarm to acknowledge", to appear on the DDU and to be vocalized.

10-Digit Keypad and Display (L, M)

The 10-Digit Keypad (L), figure 14, is the means by which the GCS Operator may enter numerical data into the system. This data may represent either the zone number to which the Operator wishes to attach the CCTV Monitors and the switchable Controls or the identification number of the GCS Operator who wishes to $\log$ on or to log off duty. A CLEAR Key is provided to erase incorrect entries. 


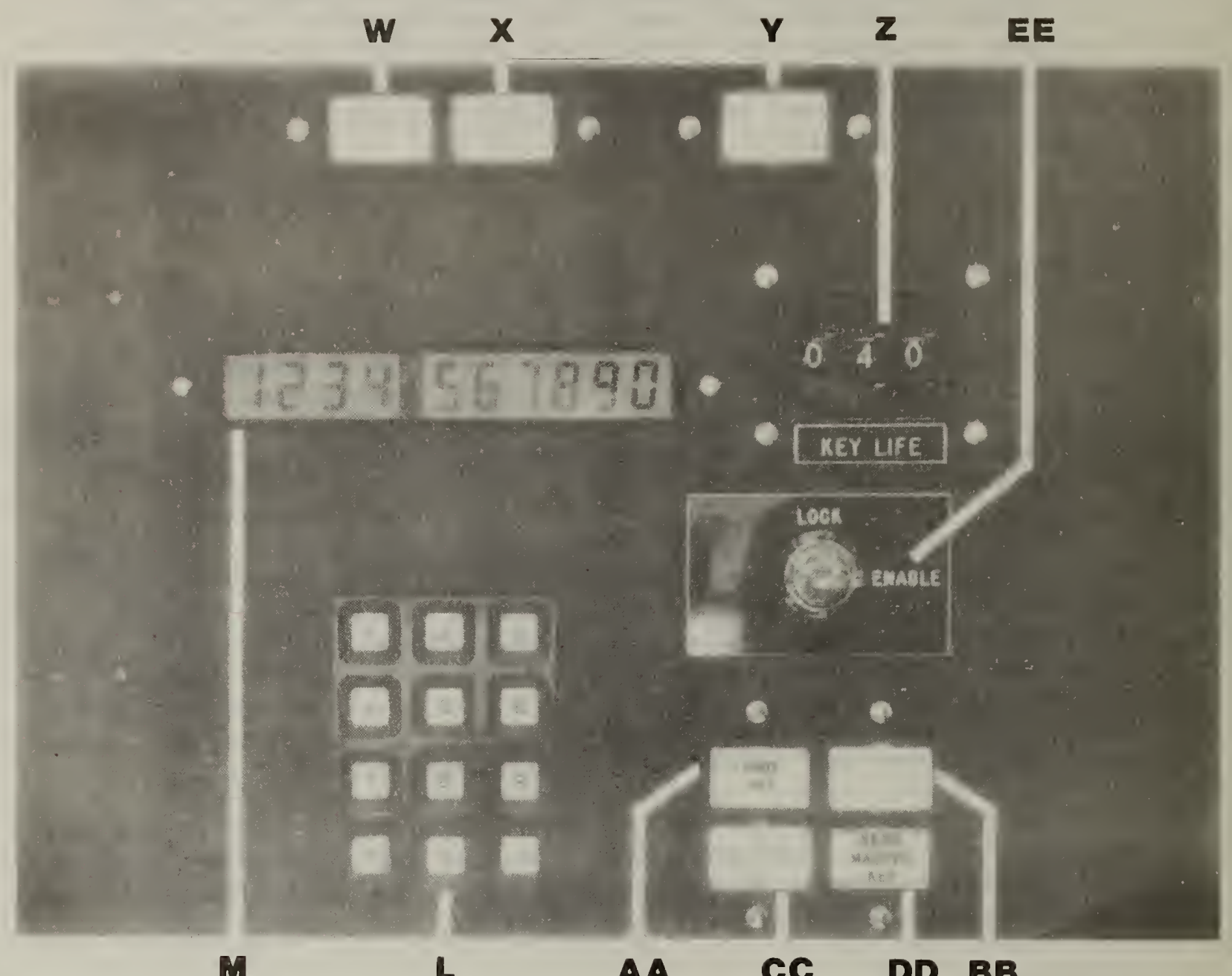

Figure 14. Right-hand control group

Associated with the 10-Digit Keypad is an LCD 10-Digit Display (M). As a number is entered via the Keypad it appears on the Display. That is, it is appended onto the right of the previously entered digits. The number remains on the Display until it is either erased by use of the CLEAR Key or entered into the system by use of the SELECT ZONE Control (N) or the LOGIN (W) Or LOGOUT (X) Controls 
The SELECT zONE Control, figure 13, is a lighted, momentary action, push switch. It is used in conjunction with the Ten Digit Keypad. If a valid zone number is entered into the Ten Digit Display via the Keypad then the CCTV Monitors and switchable controls may be attached to that zone by the actuation of the SELECT ZONE control.

If there is no zone number in the Ten Digit Display prior to actuating the SELECT ZONE Control then a Guidance Message, "No Zone Number Entered", is displayed on the DDU and is vocalized by the voice Unit. Similarly, if an invalid zone number is entered into the Ten Digit Display then the Guidance Message "Invalid zone Number selected" is used.

This Control has no effect while an unacknowledged alarm condition exists. The ACKNOWLEDGE control will continue to flash for attention. Also, if attempts are made to attach Controls to a zone that is experiencing a communication loss, a warning message is issued.

Zone Selected Display (0)

The Zone Selected Display, figure 13, is a three digit LCD. It identifies to the GCS Operator the number of the zone to which the switchable controls are attached. This number will be the same as the number in the LCD display (E) associated with CCTV Monitors to which the MPS points.

CCTV Controls (P, Q, R)

Three controls are provided on the Console to operate those assessment cameras which are fitted with remote control facilities. There is a single Pan/Tilt/Zoom Control (P), figure 13, and two individual switches (Q and $R$ ), figure 15 , for operating the focus and ir is motors.

The Pan/Tilt/Zoom Control (P) is a "T" shaped control with three degrees of freedom. Pushing it straight forward causes the camera lens to zoom in making it appear as though the camera were moving closer to the subject. Pulling the Control straight back causes the lens to zoom out 
from the subject. Rotating the top of the control to the left or right causes the camera to pan to the left or $r$ ight respectively. Rocking the top of the control forward causes the camera to tilt down. Rocking the top of the control backwards causes the camera to tilt up. The hand motions on the "T" simulate the motions which would be used if a camera were held in the Operators hand, i.e., pushing and pulling for moving close or away, rotating left and right for panning left and right, and rocking forward and back for tilting down and up.

The Pan/Tilt/Zoom Control is spring loaded and when released will return to the center, neutral position. This causes all camera motion to cease. The camera, therefore, will remain in its current position when the control is released and will remain in that position until intentionally moved.

The IRIS (Q) and FOCUS (R) Controls, figure 15 , are lighted, three position, spring loaded, center off switches. These switches are used to drive the motors for these functions. Pushing the switch causes the motor to run in one direction while pulling causes the motor to run in the opposite direction. These Controls are lighted when there is an operational camera equipped with these remote controls at the selected zone.

TV OK/Bad (S)

This Control, figure 15, is a lighted, three position, spring loaded, center off switch. It is used by the operator to tell the system when a camera at the currently selected zone is malfuncting. When the control is moved into the BAD position, the system is informed of a malfuncting camera. That camera is then considered to be not available. The CCTV Monitor display (F) will then light the "NO CAMERA" legend. If not already on, the white site status summary Display legend (I), "REPAIRS REQUIRED", will also be lighted. In addition, an entry will be made in the DDU status display showing the time and the zone at which the TV has been determined to be malfunctioning.

When the camera has been returned to functional status, that fact may be indicated to the system by the operator moving this control to the OK position. This will cause the "TV Bad" entry to be removed from the DDU status display and the CCTV Monitor display will show the Operator what 


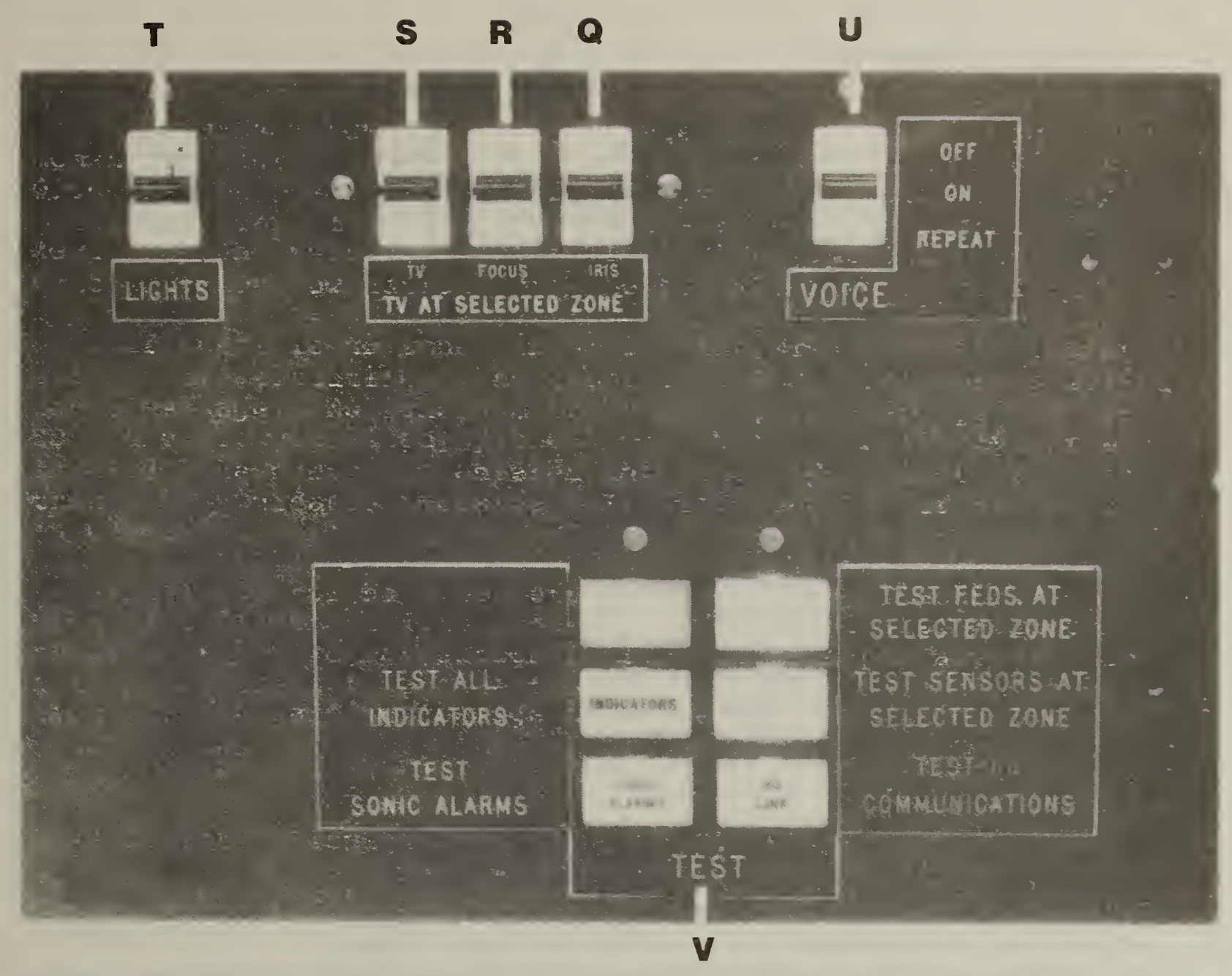

Figure 15. Left-hand control group

controls, if any, are available. Also, if there are no other devices at the site requiring maintenance then the white Site Status Summary Display legend, REPAIRS REQUIRED, will be turned off.

If the camera at the selected zone is already in the state to which the TV OK/Bad Control is being pushed, then nothing will occur. If there is no camera at the selected zone, then operation of this control will have no effect. 
This Control, figure 15, is a lighted, three position, spring loaded, center off switch. It is used by the GCS Operator to turn the lights on and off for the assessment camera(s) at the selected zone

Voice off/On/Repeat (U)

This Control, figure 15, is a lighted three position switch. It is spring loaded in the REPEAT position and automatically returns to the center or ON position. It allows the operator to have the last voice message that was output repeated by pushing the control to the REPEAT position. It also allows the Operator to turn off the Voice Unit by placing the Control in the ofF position. The Voice Unit may then be returned to the on state by placing the control in the oN position. When this Control is in the OFF position the light is also turned off.

Test Controls (V)

These Controls, figure 15, are all lighted push switches. Each is lighted when the state of the system will allow that test to occur. The functions of these controls are summarized in Table 3. 
TABLE 3

Test Control Functions

Control

This control may be actuated voluntari
the operator or in response to a system
request. This control is lighted when a zone
is selected which is either secure or in
Access. Operation causes the system to
sequentially test the squib bridgewire and
the voltage of the firing battery for each
FED device at that zone. If all FED test
good, then a guidance message results. If a
failure results then the guard is advised to
notify maintenance and the failure message
remains on the status Message Queue until
the condition is corrected and the FEDs are
retested.

Sensors

HQ Link
This Control may be actuated voluntarily by the Operator or in response to a system request. It is lighted when no unacknowledged alarm conditions exist and a valid zone has been selected. Operation of this Control causes the system to sequentially test each Sensor at the zone. A Sensor passes the test if, when a stimulus is applied, it goes from the quiet to the alarmed state, and, when the stimulus is removed, the sensor returns to the quiet state. If all sensors pass the test, a guidance message results. If any sensor fails, then the operator is notified and advised to post a guard at that zone and to notify maintenance. Each failed sensor is noted as a status message and remains on the status message queue until the condition is corrected and the sensor passes the test.

This Control transmits a test message over each of the redundant data links to higher headquarters. If the messages compare then the Link is considered to have passed the test and a Guidance Message results. If the messages do not compare, then a message goes on the status message queue, and remains until the condition is corrected and a successful test is completed. 
Indicators

Sonic Alarms
This Control energizes all lamps in the Console while it is depressed.
This Control causes the system to energize each of the Sonic Alarms in turn for a period of two seconds.

Login and Logout ( $W$ and $X$ )

These Controls, figure 14, are lighted push switches. They allow the Operator to either Login (W) or Logout (X) of the system. The Operator must first enter the correct identification number into the 10-Digit Display via the Keypad. When the tenth digit has been entered, the lights in these two controls begin to alternately flash. The lights are turned off when either of these controls is actuated. The identification number is automatically checked for its validity. If the identification number is correct then the Login is allowed. Similarly for the Logout, except that there can never be less than two GCS Operators on duty, and the Logout will not be allowed if this situation results.

Use of these controls is inhibited if there are any outstanding unacknowledged alarms. Operation of either Control while in this state will cause the system to reiterate the request for the Operator to "Please Acknowledge".

Scroll Control

(Y)

This Control, figure 14 , is a lighted, momentary actuated, spring return switch.

The site status messages which are displayed on the DDU may not fit onto a single DDU page. Therefore, they are kept in a buffer having a capacity of more than one page. This control allows the information in the buffer to be circularly advanced around the screen of the DDU, from the bottom towards the top.

This Control is lighted when the DDU buffer contents exceeds a single page of information, otherwise it is dark. It is always dark when site status information is not being displayed. 
Key Life (Z)

This Control, figure 14, is a three-digit thumbwheel switch. It is used to set the time interval, in minutes, during which an Electronic Key is valid. The least significant position is fixed at zero, so that the minimum increment is ten minutes. This value will be loaded into an Electronic Key along with a random number. When this time interval expires, the random number is erased from the system.

Code Key (AA)

The CODE KEY Control, figure 14, is a lighted push switch. Actuation of this Control causes a computer generated random number to be loaded into an Electronic Key, along with the time interval from the Key Life Control and the number of the zone that is currently selected.

In order to code an Electronic Key for a particular zone, the Key must be inserted into the Key port $(G)$, a non-zero Key Life must be selected, the Lock/Enable (EE) must be enabled, the switchable Controls must be attached to the desired zone, and then the CODE KEY Control actuated. Guidance messages are presented to the Operator if these conditions are not met.

The CODE KEY Control is lighted when no unacknowledged alarm conditions exist and a valid zone is selected for which an Electronic Key does not already exist. The light goes out when a Key is successfully loaded.

An Electronic Key loaded in this manner may be used only once. If it is not used within the time specified by the Key Life Control, the random number is automatically erased from the key memory.

Code Master Key (BB)

The CODE MASTER KEY Control, figure 14, is a lighted push switch. Actuation of this Control causes a computer generated random number to be loaded into an Electronic Key, along with the time interval from the Key Life Control. An Electronic Key loaded in this manner is valid for 
any zone and may be used any number of times within its lifetime.

In order to code a Master Electronic Key, the Key must be inserted into the Key port (G), a non-zero Key Life must be selected, the Lock/Enable (EE) must be enabled, and then the CODE MASTER KEY Control actuated. Guidance messages are presented to the operator if these conditions are not met.

The CODE MASTER KEY Control is lighted when no unacknowlegded alarm conditions exist and a Master Electronic Key does not already exist. The light goes out when a Key is successfully coded.

Send Key Control (CC)

The SEND KEY Control, figure 14 , is a lighted push switch. Actuation of this Control causes a previously generated random number to be sent to the currently selected zone. This is the same number that was previously loaded into an Electronic Key for use at the designated zone by operation of the Code Key Control. If no valid key exists for this zone, that is, no Key has been coded or a Key has been coded but its lifetime has expired, then nothing happens and guidance messages are provided to the Operator. If, at the zone, the random number in the Key matches the number that was transmitted to that zone, then a bolt is withdrawn from a strike and the door to the zone is unlocked. When the key is inserted, the random numbers in both the Electronic Key and computer memory are erased in order to limit the use of the key to a single time.

The SEND KEY Control is lighted whenever a zone is selected for which a valid Electronic Key is coded and no unacknowledged alarm conditions exist.

Send Master Key Control (DD)

The SEND MASTER KEY Control, figure 14 , is a lighted push switch. Actuation of this Control causes a previously generated random number to be sent to the currently selected zone. This is the same number which was previously loaded into a Master Electronic Key by use of the CODE MASTER KEY Control. If no valid Key exists that is, no 
Key has been coded or a Key has been coded but its lifetime has expired, then nothing happens and guidance messages are provided to the operator. If, at the zone, the random number in the Master Key matches the number that was transmitted to that zone, then a bolt is withdrawn from a strike and the door to the zone is unlocked. Whether or not a match occurs, the random numbers are not erased. This way the Master Key may be used again either at the same zone or at another zone.

The SEND MASTER KEY Control is lighted whenever a valid Master Electronic Key is coded and no unacknowledged alarm conditions exist.

Lock/Enable Control (EE)

This Control, figure 14, is a keyed two position switch. In order for the CODE KEY and CODE MASTER KEY Controls to be functional this control must be in the Enable position. Guidance messages are provided to the Operator when either the CODE KEY or CODE MASTER KEY is actuated while this Control is in the Lock position.

Call BAF (GG) and Call ARF \& BAF (FF)

These controls, figure 13, are lighted push switches. They are the means by which the operator may call out reinforcements to cope with an emergency situation. BAF is the back up force of off duty guards on or adjacent to the exclusion area. ARF is an augmentation force located at higher headquarters. When ARF is called, BAF is automatically called. However BAF may be called without ARF. When either of these Controls is actuated a three step sequence of sonic alarms is initiated. This is the same sequence which is initiated by a new alarm condition. Also, the CONFIRM (KK) and CANCEL (LL) Controls flash alternately.

Acutation of the CONFIRM Control terminates the sonic alarm sequence and causes the immediate execution of the CALL BAF or the CALL ARF \& BAF request. The CONFIRM Control also opens a voice communication link between the Operator and the BAF area so that the operator may provide the BAF forces with instructions or directions relative to the emergency situation. 
Actuation of the CANCEL Control terminates the sonic alarm sequence and cancels the CALL BAF or the CALL ARF \& BAF request. In this manner an accidental actuation of these Controls may be cancelled

If neither CANCEL or CONFIRM Controls have been actuated when the alarm sequence times out in 40 seconds, then, automatic callout occurs. This action is based on the assumption that the operator would actuate one of these Controls unless incapacitated.

In the event that a new alarm condition should occur during the sonic alarm sequence, then, in addition to the CANCEL and CONFIRM Controls, the ACKNOWLEDGE Control will begin to flash. Actuation of the ACKNOWLEDGE Control or the Monitor Pointing switch will acknowledge the new alarm. Termination of the sonic alarm sequence is only accomplished by use of the CANCEL or the CONFIRM Controls. During the new alarm condition use of either the CANCEL or the CONFIRM Controls will not only do their normal functions but also do the acknowlegdment of the new alarm.

The rational for this is that the purpose of the acknowlegdment is to provide evidence to the system that the Operator has not been incapacitated. Use of the CANCEL or CONFIRM Controls provides this evidence.

Arm FEDS Control (HH)

This Control, figure 13, provides the first of three step in the sequence to remotely fire the FEDS. Actuation of this control will cause the FIRE FEDS (JJ) and the SAFE FEDS (II) to begin to alternately flash. The ARM FEDS Control is lighted when a zone is selected that is in the alarm acknowledged state and the FEDS have not previously been either armed or fired.

Safe FEDS (II)

This control, figure 13, causes the FEDS to be returned to the unarmed state. It flashes alternately with the FIRE FEDS Control when the ARM FEDS Control has been properly used. Operating it at any other time will have no effect and guidance messages will be provided to the Operator. 
Fire FEDS (JJ)

This Control, figure 13, is the second of three steps in the sequence to remotely fire the FEDS. It flashes alternately with the SAFE FEDS Control when the ARM FEDS Control has been properly used. Actuation of this control causes the CANCEL and CONFIRM Controls to flash alternately. Use of this Control any time other than when it flashes will have no effect and guidance messages will be provided to the Operator.

If the FIRE FEDS Control had been used then the CONFIRM Control is the third and final step in the sequence to remotely fire the FEDS. All steps. in the sequence must be taken in the proper order to fire the FEDs: select a valid zone, Arm FEDS, Fire FEDS, and Confirm. If, at any point in the sequence, the Operator selects another zone and then returns to the original zone, then the sequence continues from where it was when the original zone was selected. That is, attaching to another zone does not return the FEDS at the original zone to the safe state.

Confirm (KK) and Cancel (LL)

These controls, figure 13, flash alternately when the FIRE FEDS or the CALL BAF or the CALL ARF \& BAF Controls are properly operated. Their use to confirm or cancel these commands has already been described.

Reset Alarm (MM)

The RESET ALARM control is shown in figure 13. This control is used to restore a zone to the secure condition when an access condition is terminated, or after conditions causing a sensor to alarm have been quieted. In addition, if an Alarm State is present and the use of the TEST SENSORS control shows that the sensor causing the alarm has failed, then, use of the RESET ALARM control will restore the zone to a secure state. Prior to restoring the zone to the Secure state under such circumstances, the Operator must insure that a guard has been posted at that zone.

If there is no Unacknowledged Alarm condition present, then the RESET ALARM control is lighted when the selected zone is in either the Access or Alarm States. 
When this control is successfully used, the zone is removed from the alarm queue and the alarm messages are replaced by status messages on the DDU.

Date Display (NN)

A four digit display of the current date as is shown in figure 1l. The first two digits represent the month. These are separated by a colon from the second two digits which show the day of the month.

Time Display (OO)

Directly below the date display, a six digit display shows the current time in hours, minutes, and seconds on a 24-hour basis. This is shown in figure 11.

GCS HARDWARE DESCRIPTION

In the following sections, some of the specially designed or modified hardware components of the GCS simulator are described. 


\section{Console Processor Interface Boards}

The Console Processor in the GCS contains six parallel line interface boards and one serial line interface board with four ports. The parallel line outputs drive the lamps, alarms, digital readouts, and the video switches; the parallel inputs are connected to the console switches. The serial line ports interface the Console Terminal, DDU, Voice Unit, and the Event Processor (download link) to the Console Processor. An additional parallel line interface was modified specifically to communicate with the Event Processor at a higher data rate than is possible with the serial download link.

A $60 \mathrm{~Hz}$ line clock is available in the Console Processor for timing and synchronization. It is not configured as an I/O device (not addressable, therefore not programmable) but is connected directly to an interrupt line reserved for that purpose.

\section{GCS Special Interface Boards}

To connect the GCS hardware to the console Processor, parallel line interface boards requires a variety of circuits for decoding, driving, debouncing, etc. This circuitry is located on 10 special-purpose logic boards mounted in a card file in the left pedestal of the GCS adjacent to the Processor. Cables from the parallel line interfaces connect to these boards as do cables from the six console panels, thus this card file serves as a junction box for-signal cables with signal routing done on the boards and on the card file backplane. The 10 logic cards are of the following types:

Switch Debounce (2 each)

Lamp Drivers ( 3 each)

LCD Decoder/Drivers ( 3 each)

Keypad/10-digit Display Interface (2 each) 


\section{Switch Debounce}

The pushbutton and lever switches on the Console require debouncing circuits so that one actuation is not misinterpreted as several due to contact bounce. This is accomplished by connecting each switch to a latch (RS flipflop); the latch is set upon closure of the normally-open switch contacts and is unaffected when these contacts bounce open. The latch is reset by the normally-closed contact pair when the switch is released and, again, the latch is not affected by bounce. Note that double-throw switches are required for this technique. The latch outputs are tied directly to the inputs of the parallel line interface boards, one line (bit) per switch.

\section{Lamp Drivers}

There are two incandescent lamps in each indicator and each illuminated switch. Each lamp requires $180 \mathrm{milliam-}$ peres of current at 5 volts. Since this is far more than the current drive capability of the parallel line interface outputs, lamp driver circuits are required. The lamp driver chips that are used have a 2-input OR-gate in front of each driver circuit which facilitates the implementation of a hardwired lamp test feature. One input of each gate is tied to an output of a parallel line interface board; the remaining inputs are tied together and driven by the TEST INDICATORS function switch so that the lamps are all turned on when this switch is depressed.

Power for the lamps comes from a 5 volt, 25 ampere supply. In order to minimize the power surge through the system cabling when all lamps are simultaneously turned on (as in TEST INDICATORS) the lamps are biased to nearly glowing when in the OFF state. This is done by connecting a 31 ohm resistor to each lamp pair in parallel with the lamp driver. This has two effects which reduce the step increase in current: (a) the power supply is pre-loaded to more than $50 \%$ of its peak load, and (b) the lamp filaments are preheated which increases their resistance. This reduces inrush current when the lamp is turned on. The lamps are rated for 6.3 volt operation and running them at only 5 volts greatly increases their life expectancy. 
The liquid crystal displays (LCD's) are one-half inch high, seven-segment digits with four or six digits per display package. To drive each segment individually from the Processor ports would require a very large number of lines (bits), that is, seven times the number of digits. Therefore, decoder/driver integrated circuits are used which require only four input lines per digit. still, to drive the 19 digits the GCS uses would take 76 bits of parallel port output, a formidable number. A further reduction is accomplished by using a multiplexing scheme. This scheme requires only 16 bits for data plus 6 bits to select the display to be set or updated. The decoder/driver chips contain latches for the data and all the driver circuits necessary for supplying the required waveforms to the ICD backplanes and segments.

Keypad/10-digit Display

The 12 keys of the keypad are not interfaced to the Console Processor through the parallel ports. Since the keypad output is always directed to the 10-digit display, it has been hardwired for that function. Eleven of the single-pole, single-throw switches on the keypad are debounced and then encoded to four lines. These four lines feed a $10 \times 4$-bit shift register. As each key is depressed, its number is entered into the rightmost digit position of the register, causing previous entries, if any, to shift to the left. When the register is full (10 digits entered) a FULL status signal is asserted at one of the parallel ports.

The Processor reads one digit at a time; the leftmost digit. This causes the string to shift left one position, with blank spaces coming in on the right end. The process of read/shift is repeated until the register is empty (all blanks).

The eleventh key on the pad, labeled "*" , inserts a "-" symbol in the display, but this capability is not normally used. The remaining (12th) key on the keypad is used to manually clear the register. This is used when the operator realizes he has made an erroneous entry. The clear key is not decoded; it simply forces the code for "blank" into all digit positions. 
The contents of the shift register is at all times displayed by the 10-digit LCD display. Non-latching decoder/driver circuits translate the four-line codes in the shift register to the seven-segment LCD digits.

For error conditions, the display can be placed in a special mode which causes it to flash a pattern of dashes (--------). Upon receipt of an ERROR command from the Processor, codes for "blank" and "-" are alternately forced into all stages of the shift register at a rate of about 2 Hz. The flashing continues until the Processor clears the ERROR bit or the CLEAR key is depressed.

\section{Monitor Pointing Switch}

The Monitor Pointing Switch (MPS) is a large 2-position switch designed to give unambiguous and rapid switching of CCTV displays and associated controls. Reliable electrical switching, positive mechanical action, and large actuator size are the prime requisites of this custom designed switch.

Electrical switching is done with optical switches instead of mechanical contacts. Each optical switch consists of an LED source and photo-transistor detector molded into a U-shaped package. A flange on the underside of the MPS passes through the "U" to obstruct or pass the light beam according to the position of the switch. Two optical switches are used for each position for redundancy; the outputs of the detectors are OR-gated so that one failure will not cause the MPS to be inoperative.

Outputs from the MPS to the Console Processor consist of three bits: MPS LEFT, MPS RIGHT, and MPS MOVED. The MPS MOVED bit is asserted by motion in either direction and is cleared when the processor reads that information. Connections to the parallel interface lines of the processor are made via one of the switch debounce boards. 


\section{CCTV Subsystem}

The CCTV Subsystem employes commercial 525 line resolution, black and white camera and monitor equipment. In virtually all other respects, however, the system described below is quite different from what would be installed at an actual site. A site with a large number of cameras would require a more sophisticated Video Switcher and would employ video transmission and camera control arrangements of the sort described in Reference 1.

Cameras

In this simulation, four CCTV cameras are used in the alarm assessment system. Three are stationary, fixed lens cameras; the fourth is mounted on a pan/tilt head and has a zoom lens with controllable iris and focus adjustments.

\section{Video Switcher}

The video signals from the cameras are connected to the monitors by the Video Switcher. Switching is accomplished through reed relays that are in turn controlled by the Console processor through one of the parallel line ports. Two bits of the parallel port are assigned to each video signal to select left or right display. The Processor, using internal information and the Monitor Pointing Switch position, actuates the appropriate relay(s). The top/bottom assignment is hard-wired since this depends on the number of cameras per zone. For two-camera zones the video from the camera with the longer focal length is switched only to top monitors; all other video is switched to bottom monitors.

\section{Camera Controls}

Pan, tilt, and zoom control for the camera equipped with those features is done via a special handgrip style control. Electrically, the Pan/Tilt/Zoom Control consists of optical switches, two switches for each control axis. Each optical switch has a light source and a detector molded into one package. The light paths of the switches are all obstructed when the control is in the rest position; actuation allows light to pass in the selected switch. With this design, failure of a light source will not result in unintended motion of the camera. 
Control of lens iris and focus is provided for by two 3-position lever switches mounted on the lower left panel. These are conventional hard contact switches.

\section{Camera Motor Drive}

The controllable CCTV hardware used in this simulation is part of a commercial area surveillance system that has been adapted for this application. The original control module is mounted in the right pedestal of the GCS. The GCS camera controls are connected through relays to this control module; the control module is in turn cabled to the camera. The control module contains the required power supplies of 165 volts and + and -70 volts $D C$. It is designed to drive camera positioning equipment at distances up to 3000 feet.

\section{Voice Unit}

The Voice Unit in the GCS is a stored vocabulary speech response device that synthesizes utterances from encoded, compressed speech data that has been stored in read-only memory (ROM). The vocabulary has been selected for the application and is sufficient to annunciate all the messages that are printed on the DDU.

The Voice Unit is computer controlled through the use of word pointer and commands. Word pointers specify which utterance should be spoken; commands are used to instruct the unit to change pitch, select frame rate, set interutterance delay, etc. Commands and word pointers are arranged in the desired sequence by the computer program and downloaded to a buffer in the Voice Unit via a serial $I / O$ port. Once they have been downloaded, a microcontroller in the unit retrieves the encoded speech data from ROM and presents it to the speech synthesizing circuits that generate the digital representation of the original speech waveform. A digital-to-analog converter then transforms this representation into its analog counterpart which is amplified and fed to a conventional 8 ohm speaker.

The vocabulary may be changed or added to by changing or adding ROM chips. Seven 28-pin ROM sockets are available to the user. These sockets accept 16,32 , or 64 kilobit ROMs, thus the vocabulary memory may range from 16 kilobits to 448 kilobits. (One second of speech requires about 2.2 kilobits of memory.) The present implementation uses all seven ROM sockets for a total of 248 separate utterances. These utterances include 226 discrete words, 11 prefixes and 
suffixes, 2 phrases of 5 words each, and 4 tones. Not all ROMs are utilized to capacity because the vocabulary was ordered in increments as the system was developed instead of as a single unit. For example, one ROM contains just two phrases (in German); this was done to demonstrate the multi-lingual capabilities of the unit.

Sonic Alarms

Three audible alarms are provided. They were selected so that each is easily distinguishable from the other. Two are solid state audio devices; one gives a $2900 \mathrm{~Hz}$ pulsating sound and the other a $4500 \mathrm{~Hz}$ continuous tone. The third device is a conventional buzzer. The alarms are driven from one of the parallel ports of the Console Processor in the same manner as the lamps, i.e., one bit of the port drives each device through a current driver circuit. All of the devices are mounted on the upper left panel of the GCS.

\section{Console shell}

The cabinetry for the GCS has been designed for flexibility of device placement in order to facilitate experiments in the layout of controls and displays. The Console consists of an upper plywood shell, with cutouts for panels and shelves for TV monitors. It rests on two short equipment racks which serve as pedestals.

The plywood shell is 34 inches long by 68 inches deep. The lower portion, sloped 15 degrees from the horizontal plane, has a long cutout designed to accept three $11.5 \times 20$ inch metal panels. The upper portion, sloped 15 degrees from the vertical plane, has five recessed shelves for placement of the four CCTV monitors and the DDU. Shelves are used here instead of rigid mounting devices to facilitate experiments with angular and depth positioning of these units. In addition to the shelves, there are cutouts for three metal panels: $8 \times 24$ inch panels on either side of the shelf area and a $5 \times 18$ inch panel at top center. All exterior surfaces of the plywood shell, including the recessed shelves, are covered with Formica.

The pedestals are stock equipment racks, 26 inches high, 22 inches wide, and 28 inches deep. The internal mounting space accepts standard 19 inch wide panels. The left pedestal houses the Console processor, the card file for the special GCS interface boards, and two power 
supplies. The Processor is mounted on chassis slides; the card file is mounted to the front rails above the processor. The power supplies are attached to the rear rails. The right pedestal contains the Voice Unit, Video Switcher, and Camera Motor Drive.

\section{Cabling}

GCS internal cabling is designed around a modular concept for ease of assembly and maintenance. Each panel may be lifted out, quickly disconnected, and completely removed from the console. All signal connections are made via flat cables; cable ends have crimp-on insulation displacement connectors. DC power ( +5 volts) is brought to each panel via a locking circular connector which accepts up to 14-guage wire.

\section{Power Supplies}

Five volt DC power is required for the special interface boards and for the lamps. Since the lamp power supply is subject to large step changes in load it is inadvisable to run the lamps and interface boards from the same supply. Hence, two supplies are used, a 25 ampere switching supply for the lamps and a 3 ampere linear supply for the interface boards. The processor and other packaged units such as the Voice Unit, CCTV cameras, etc., have their own internal DC supplies.

\section{SIMULATION SOFTWARE}

For the sake of expediency and economy, the selection of hardware and software was based on availability and familiarity rather than optimization of function and speed. The hardware used existing available DEC LSI $11 / 23$ [*]

[*] Certain commercial equipment, instruments, or materials are identified in this paper in order to adequately specify the experimental procedure. Such identification does not imply recommendation or endorsement by the National Bureau of standards, nor does it imply that the materials or equipment identified are neces- 
processors. The software was written in the C language [3] (and some assembly language where necessary). The UNIX operating system served as the development system environment, and was also used for part of the run time environment.

Software Design

Three cooperating processes were the basis of the software design. One, the Console Process, was to service the console inputs and outputs, and the second, the Event Process, was to service and maintain the history of events from the individual zones via the C.U. and from the console Process. A third process, the Director Process, was also required to coordinate events and simulate the actions that could occur at the various zones.

Each process was to be placed on a separate processor, primarily because the processors available had a limited per process address space (56K bytes/process). Also, project requirements specified that the Console be polled rather than interrupt driven, to increase speed and avoid operating system overhead. Placing each process on a separate processor allowed the Console process to run stand-alone in a polling mode, while the other processes could be run under an interrupt driven operating system. This was possible, for this feasibility model only, since time was not as critical for these other processes. The Director Process was placed on a third processor, mainly so as not to interfere with or delay the other processes.

The additional communication overhead between the processors was more than offset by the gain in speed with parallel processing. The communication between the three processes was designed to be message based, with an extensible message set. It was inplemented on a 16 bit parallel port for speed, approximately $40 \mathrm{~K}$ bytes/sec.

sarily the best available for the purpose. 
The Console process is a combination of a minimum operating system and an application program running on a bare processor. It functions mainly as an intelligent device driver. It samples the state of the console switches, processes any change in their state, exchanges messages with the Event process, and updates the console display lights to reflect current operational conditions. Messages are exchanged to inform the Event process of any state changes, as well as accepting information on current operational conditions from the Event Process. The Console Process also maintains an abbreviated status record about the overall site and the currently attached zone, if any. Formatted DDU messages, generated by the Event process, are stored and displayed as necessary by the Console process. The software organization of the Console Process is shown in figure 16, where each box represents a module. The device drivers, scheduler, and clock modules handle the necessary operating system features in this polling environment. The remaining modules handle the various application features. The only requirement placed on a module is that once it starts executing it completes its designated function.

The Scheduler

In this organization the execution flow is controlled by a table driven scheduler which continually polls each application module in sequence. The individual modules determine if there is any work for them to do, and at completion return a result indicator to the scheduler. Based on the result indicator the scheduler determines if one or more of the next sequential application modules should be skipped during this round of polling. After the polling of any application module the device driver module is always polled next. Thus, in effect inter leaving the device driver module between each application module.

The Device Driver

This module handles the details of getting data between the buffers and the individual devices (DDU, Logging Printer, Voice Unit, and Communication Channel - inbound and outbound). Each device is allocated a dedicated circular buffer. The inbound communication channel acts as a 


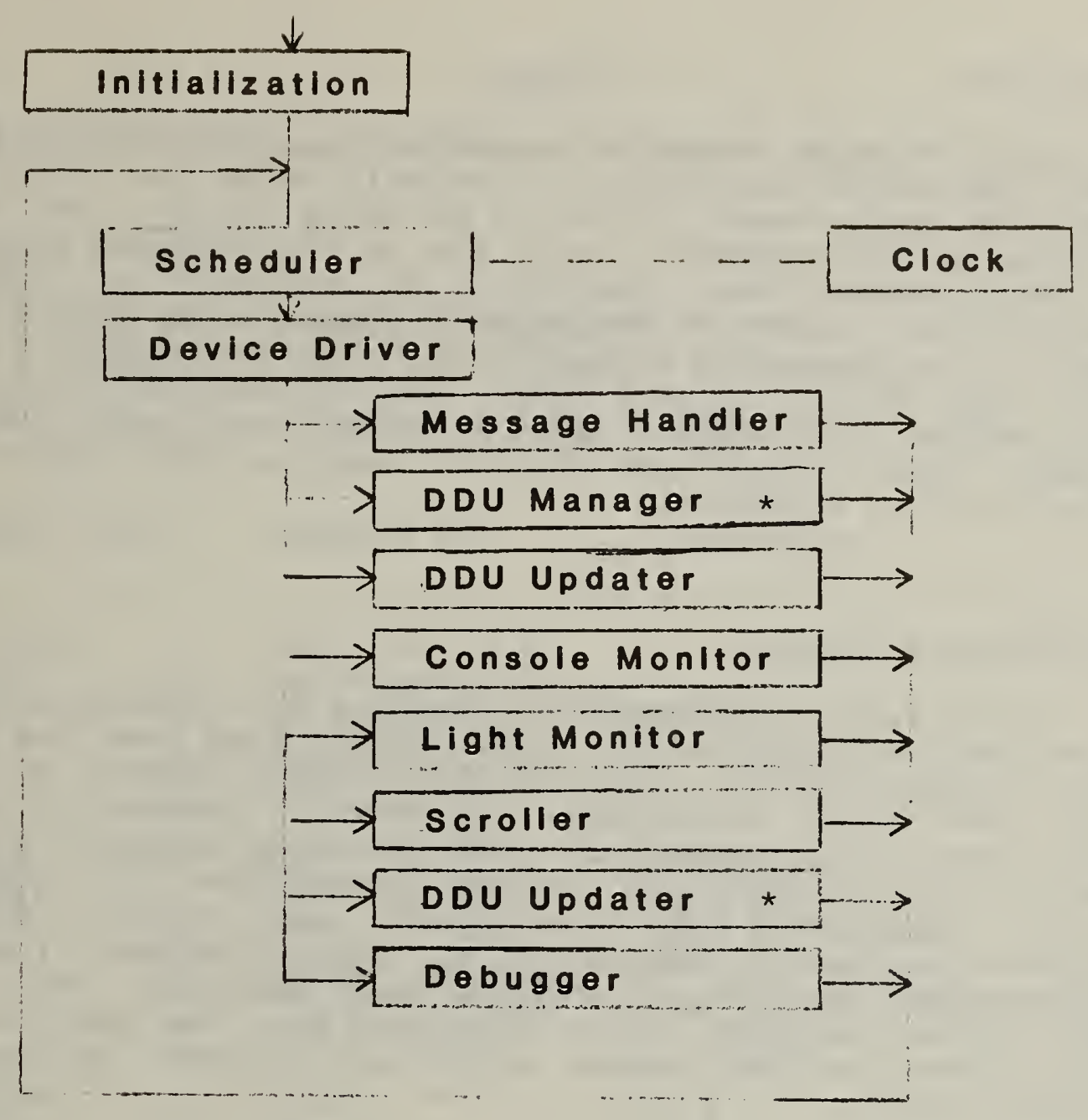

*Executed more than once for a polling sequence, but only a single copy of code is used.

Each rectangle represents a module.

Figure 16. Block diagram of the Console Process producer function, filling its buffer, while for all the other devices the drivers act as a consumer function, emptying their buffers. 
The Clock

This module, which is a combination of operating system and application function, is the only exception to the noninterrupt requirement. This is strictly due to the architecture of the processor which had no programmable interface to the hardware clock. Therefore, in this case an interrupt was the only means of determining when a clock tick had occured. The operating system function of this module is to keep track of the current time. The application function of this module is to update the console date and time LCD display each second and maintain various other application required time durations.

The Message Handler

This application module processes all incoming messages from the Event Process and then removes them from the incomming message buffer. The list of messages between the Console and Event Processes are shown in figures 17 and 18, along with their parameters. The incoming messages of figure 18 fall into two general categories: (1) update the current abreviated site or attached zone status, and (2) process a device request (i.e., add or delete a formatted DDU message, execute a formatted voice message, etc.). If the current message is not concerned with the DDU, then the DDU Manager and DDU Updater module are skipped during this polling cycle. 
TABLE 4

List of Messages from Console Process to Event Process

Message format:

\begin{tabular}{|c|c|c|c|}
\hline $\begin{array}{c}\text { size in } \\
\text { bytes }\end{array}$ & $\begin{array}{c}\text { command } \\
\text { type }\end{array}$ & $\begin{array}{c}\text { command } \\
\text { number }\end{array}$ & parameters \\
\hline 2 bytes & $\frac{1}{1}$ byte & I byte & n bytes \\
\hline
\end{tabular}

COMMAND TYPE COMMAND NO. PARAMETERS

\begin{tabular}{|c|c|c|c|}
\hline $\begin{array}{l}\text { request } \\
\text { info }\end{array}$ & $\begin{array}{l}0 \\
1 \\
2 \\
6 \\
9\end{array}$ & $\begin{array}{l}\text { - date and time } \\
\text { - valid zone list } \\
\text { - zone status } \\
\text { - alarmed status } \\
\text { - site status }\end{array}$ & $\begin{array}{l}- \\
\text { zone } \# \\
-\end{array}$ \\
\hline $\begin{array}{l}\text { send } \\
\text { info }\end{array}$ & $\begin{array}{l}0 \\
2 \\
3 \\
4 \\
7 \\
8\end{array}$ & $\begin{array}{l}\text { - date and time } \\
\text { - zone status } \\
\text { - CCTV for zone \# } \\
\text { - lights, zone \# } \\
\text { - set FEDs state } \\
\text { - acknowledge }\end{array}$ & $\begin{array}{l}\text { date, time } \\
\text { zone status } \\
\text { zone } \#, \text { ok/bad } \\
\text { zone } \#, \text { on/off } \\
\text { zone } \#, \text { safe/arm/fire } \\
\text { - }\end{array}$ \\
\hline $\begin{array}{l}\text { guard } \\
\text { action }\end{array}$ & $\begin{array}{l}0 \\
1 \\
3 \\
4\end{array}$ & $\begin{array}{l}\text { - } \operatorname{login} \\
\text { - logout } \\
\text { - code key } \\
\text { - code master }\end{array}$ & $\begin{array}{l}\text { ID \# } \\
\text { ID \# } \\
\text { zone\#, key } \frac{\# \text {, key life }}{\text { key\#, key life }}\end{array}$ \\
\hline $\begin{array}{l}\text { test } \\
\text { request }\end{array}$ & $\begin{array}{l}0 \\
1 \\
2\end{array}$ & $\begin{array}{l}\text { - test FEDS } \\
\text { - test sensors } \\
\text { - test HQ links }\end{array}$ & $\begin{array}{l}\text { zone \# } \\
\text { zone \# } \\
\text { - }\end{array}$ \\
\hline $\begin{array}{l}\text { request } \\
\text { help }\end{array}$ & $\begin{array}{l}0 \\
1 \\
2\end{array}$ & $\begin{array}{l}\text { - BAF } \\
\text { - ARF } \\
\text { - restore }\end{array}$ & $\begin{array}{l}\text { zone \#, guard/automatic } \\
\text { zone \#, guard/automatic } \\
\text { - }\end{array}$ \\
\hline access & $\begin{array}{l}0 \\
1 \\
2 \\
3\end{array}$ & $\begin{array}{l}\text { - access } \\
\text { - master access } \\
\text { - clear access } \\
\text { - clear master } \\
\text { access } \\
\text { - secure } \\
\text { - check key life }\end{array}$ & $\begin{array}{l}\text { zone\# } \\
\text { zone\# } \\
\text { zone\# } \\
\text { zone\# } \\
\text { zone\# } \\
\text { - }\end{array}$ \\
\hline
\end{tabular}




\section{TABLE 5}

List of Messages from Event Process to Console Process Message format:

\begin{tabular}{|c|c|c|c|}
\hline $\begin{array}{c}\text { size in } \\
\text { bytes }\end{array}$ & $\begin{array}{c}\text { command } \\
\text { type }\end{array}$ & $\begin{array}{c}\text { command } \\
\text { number }\end{array}$ & parameters \\
\hline 2 bytes & -1 byte & I byte & n bytes \\
\hline
\end{tabular}

COMMAND TYPE COMMAND NO. PARAMETERS

\begin{tabular}{|c|c|c|c|}
\hline $\begin{array}{l}\text { request } \\
\text { info }\end{array}$ & $\begin{array}{l}0 \\
2\end{array}$ & $\begin{array}{l}\text { - date and time } \\
\text { - zone status }\end{array}$ & zone \# \\
\hline $\begin{array}{l}\text { send } \\
\text { info }\end{array}$ & $\begin{array}{r}0 \\
1 \\
2 \\
6 \\
9 \\
10 \\
11\end{array}$ & $\begin{array}{l}\text { - date and time } \\
\text { - valid zone list } \\
\text { - zone status } \\
\text { - alarm zone status } \\
\text { - site status } \\
\text { - communication } \\
\text { failure } \\
\text { - alarm }\end{array}$ & $\begin{array}{l}\text { date, time } \\
\text { zone \#'s } \\
\text { zone status } \\
\text { zone status } \\
\text { site status } \\
\text { - } \\
\text { zone status }\end{array}$ \\
\hline guard & 0 & - identity valid & yes/no \\
\hline test & 2 & - HQ link & - \\
\hline $\begin{array}{l}\text { access } \\
\text { results }\end{array}$ & $\begin{array}{l}0 \\
1 \\
2 \\
3 \\
4 \\
5\end{array}$ & $\begin{array}{l}\text { - regular key } \\
\text { - master key } \\
\text { - clear access } \\
\text { - clear master } \\
\text { - go secure } \\
\text { - continue alarm }\end{array}$ & $\begin{array}{l}\text { zone\#, yes/no } \\
\text { zone\#, yes/no } \\
\text { zone\# } \\
\text { zone\# } \\
\text { zone\# } \\
\text { zone\# }\end{array}$ \\
\hline $\begin{array}{l}\text { logging } \\
\text { message }\end{array}$ & $\begin{array}{l}0 \\
1\end{array}$ & $\begin{array}{l}\text { - status } \\
\text { - alarm }\end{array}$ & $\begin{array}{l}\text { message } \\
\text { message }\end{array}$ \\
\hline $\begin{array}{l}\text { DDU } \\
\text { message }\end{array}$ & $\begin{array}{l}0 \\
1 \\
2 \\
3 \\
4 \\
5 \\
6 \\
7\end{array}$ & $\begin{array}{l}\text { - remove status } \\
\text { - remove alarm } \\
\text { - display status } \\
\text { - display alarm } \\
\text { - display new alarm } \\
\text { - augment new alarm } \\
\text { - guidance } \\
\text { - canned guidance }\end{array}$ & $\begin{array}{l}\text { message \# } \\
\text { message \#, zone\# } \\
\text { message \#, message } \\
\text { message \#, zone\#, message } \\
\text { message \#, zone\#, message } \\
\text { message \#, zone\#, message } \\
\text { message \#, guidance message } \\
\text { message (guidance)\# }\end{array}$ \\
\hline $\begin{array}{l}\text { voice } \\
\text { message }\end{array}$ & $\begin{array}{l}0 \\
1\end{array}$ & $\begin{array}{l}\text { - status } \\
\text { - alarm }\end{array}$ & $\begin{array}{l}\text { message } \\
\text { message }\end{array}$ \\
\hline
\end{tabular}


The Message Handler and the DDU Manager

A major function of the Console Process is to maintain a data base of current DDU messages, and manage their presentation. This requires a reasonable amount of work and has resulted in this function being split into a number of modules. The Message Handler module does the initial processing of a DDU message request, by updating the actual DDU data base and marking the resulting status. The DDU Manager module acting on the new data base status then computes the contents of a new display window for the DDU, and the changes required to the current display window to produce the new display window.

The DDU Updater

This application module is the final step of the DDU function. The DDU data base consists of two separate, but similar, data bases: (1) a status message data base, and (2) an Alarm message data base. The DDU Updater module keeps track of which data base is currently being displayed. If a change is indicated in the data base to the current DDU display, the DDU Updater module fills the DDU buffer with the previously computed changes required to produce the new display window.

The Console Monitor

This module samples the console switches and processes any newly activated ones. The processing starts by checking the validity of the activated switch against the current system state. If it is not valid, then some combination of an audio beep, a printed DDU guidance message, and an audio guidance message will provide the operator error feedback. If the activated switch is valid then the indicated action is initiated by updating the local abreviated status information and sending the appropriate messages to the Event Process.

The Light Monitor

Based on changes in the local status and data base this module turns on, off, or flashes the lamps in the console switches and indicators. 
The Scroller

This is another module related to the presentation of the DDU message data base. It is applicable to the condition of too many messages to fit onto the display window at one time. When activated this module will compute the contents of a new display window that is the current display window circularly scrolled up by a single, but entire, message. Only entire messages are displayed, never any partial messages. When successfully activated this module indicates to the scheduler that the DDU Update module should be the next application module to be polled.

The Debugger

This module was used during debugging and is intended for normal preventative maintenance and trouble shooting. It interacts with a separate terminal, that may be built in or a portable one that can be plugged in when required. It allows maintenance personnel to interactively peruse the local data base and status information, monitor the contents of messages exchanged with the Event process, as well as run predefined diagnostics while the system continues to work.

\section{The Event Process}

The Event Process maintains a detailed data base for all zones within the site. This data base contains information on the state of each zone (i.e.,Secure, Alarmed, Accessed, etc.), the state of each sensor and FEDS, as well as a reference to all currently active DDU messages residing within the DDU message data base maintained by the Console Process.

For this simulation model the Event process was implemented within an interrupt driven operating system environment, rather than a stand alone polling environment as was the Console Process. Under this type of environment, when a process issues a read device request, the process is usually blocked until the read request is completed. When the device is a terminal or a communication link to another process, the duration of this blocking can be very long, depending on whether the other end has something to write. The Event process must wait for asyncronous input from the 
Console Process, the Director Process, and from a local debugging terminal. Therefore, the existing blocked I/O of the operating system was not acceptable. A modification was made to the applicable device drivers which provided a programable user option to invoke non-blocked device reading. Under this option, a read request would complete immediately with an indication whether or not there was something to be read. This modification provided the Event Process the capability to poll each of these devices and to determine when there was something to process.

A block diagram of the software organization for the Event Process is shown in figure 19, where each box represents a module. The Controller polls the communication channel to the Console Process, the Director Process, and to the Debugging terminal. When a message is received, the Control module passes it to the appropriate handler module for processing.

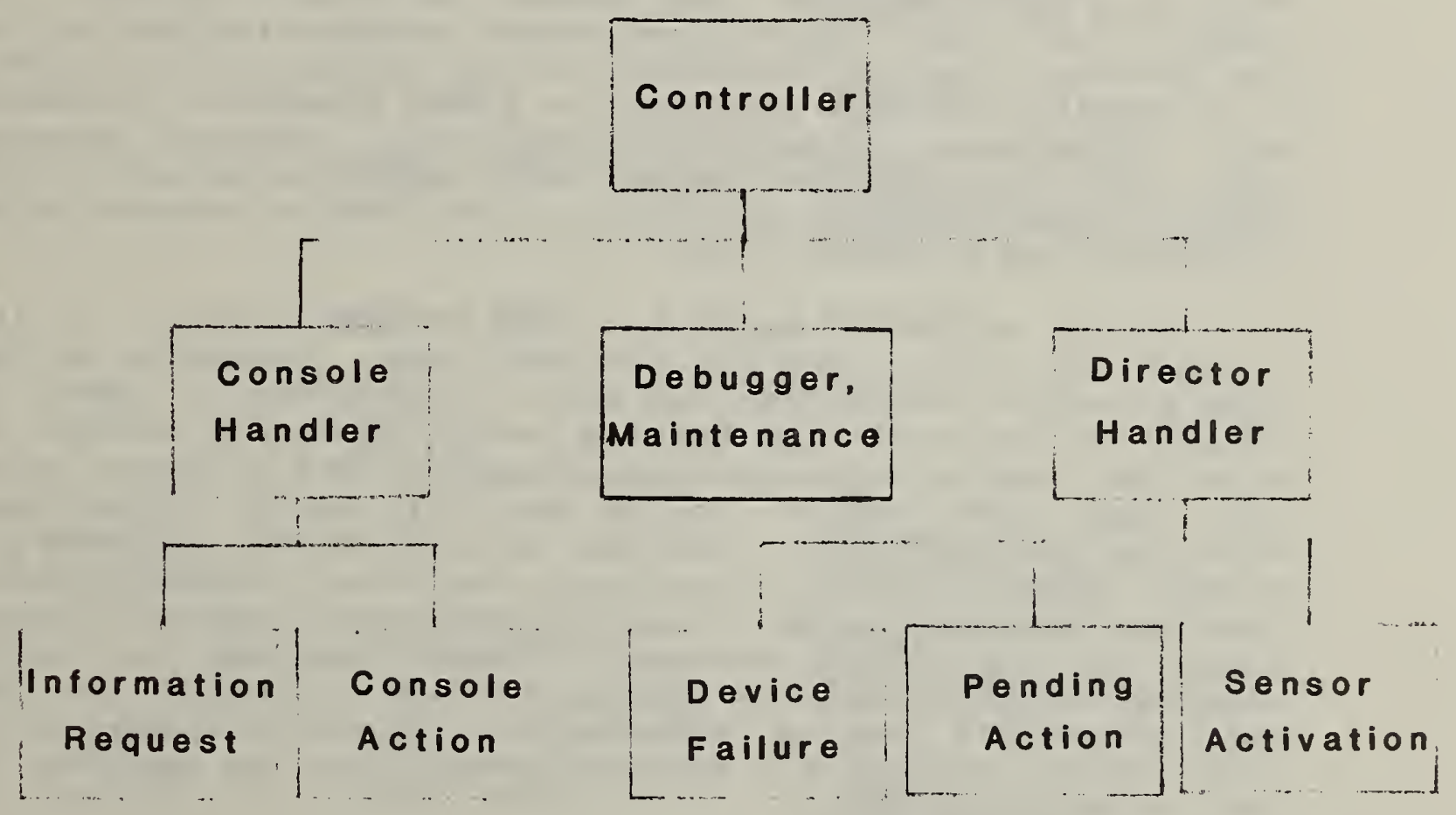

Figure 17. Organizational block diagram of Event Process software. Each rectangle represents a module. 
Console Handler

The Console Handler module processes messages from the Console process. These messages fall into two general categories (1) request for specific information from the Event Process data base and (2) notification of a legal console action.

The first category of Console Process messages, request information, is relatively straight forward. This category includes messages such as "request list of valid zones", "request abreviated status of zone 15", etc. The requested information, located within the Event Process' data base, is extracted and sent to the Console Process.

The second category of Console Process messages, action notification, is the more complicated category (e.g., fire FEDS at zone 4 ). This requires the Event process to check the validity of the action for the current system state. If it. is not valid, then either a reject message or a specific message is returned to the console process. A reject message is a short message, that causes the Console process to update its local abreviated status information and inform the Operator that the requested action failed. Alternatively a specific message requires the Event Process to assemble both a completely formatted DDU and audio guidance message that will provide the Operator with specific rejection details. These messages are sent to the Console process which maintains and presents them.

If the action is valid then the indicated action is initiated by first updating the data base. Depending on the type of action requested, the Event process may be able to affect or provide the desired result by itself. If not it must then send an appropriate messages to the Director process and then, while noting that this action is pending, wait for the response. When the results of the action are known, either success or failure, the Event process updates its date base and sends a result message and specific messages to the Console Process. A result message is a short message, which causes the Console process to update its 10cal abreviated status information. Specific messages, as noted above, provide the Operator details on the results of the requested action.

It should be noted that "determining system state", "updating the data base", and "assembling formatted messages" are complex operations that involve significant workload. While the Event process is servicing these various requests, other actions and requests may be processed in parallel by both the Console and the Director Process. 
TABLE 6

List of Messages from Director Process to Event Process

Message format:

\begin{tabular}{|c|c|c|c|}
\hline $\begin{array}{c}\text { size in } \\
\text { bytes } \\
\text { I byte }\end{array}$ & $\begin{array}{c}\text { command } \\
\text { type }\end{array}$ & $\begin{array}{c}\text { command } \\
\text { number }\end{array}$ & parameters \\
\hline I byte & I byte & n bytes \\
\hline
\end{tabular}

COMMAND TYPE COMMAND NUMBER

PARAMETERS

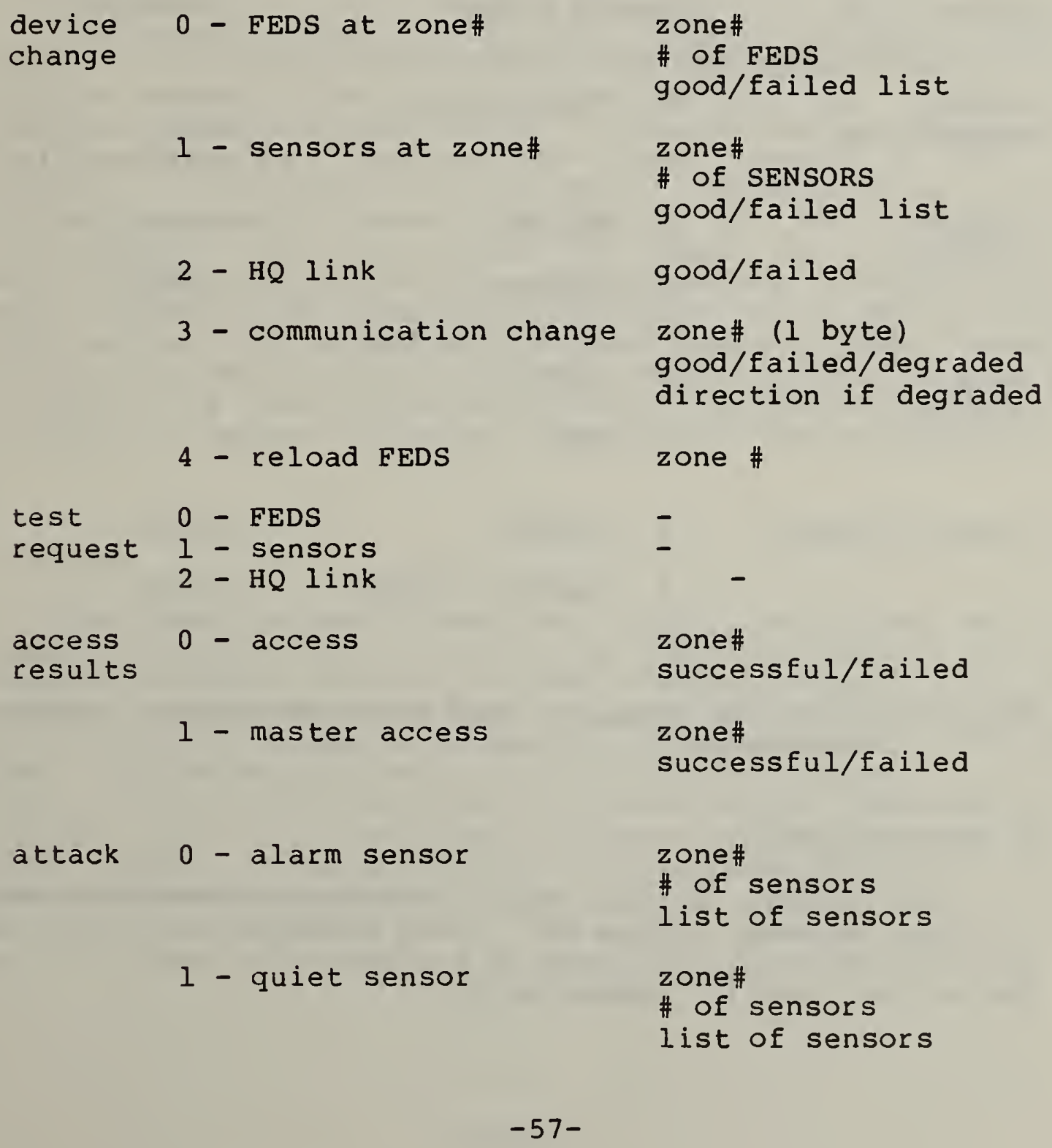


TABLE 7

List of Messages from Event Process to Director Process Message format:

\begin{tabular}{|c|c|c|c|}
\hline $\begin{array}{c}\text { size in } \\
\text { bytes }\end{array}$ & $\begin{array}{c}\text { command } \\
\text { type }\end{array}$ & $\begin{array}{c}\text { command } \\
\text { number }\end{array}$ & parameters \\
\hline I by te & I byte & I byte & $n$ bytes \\
\hline
\end{tabular}

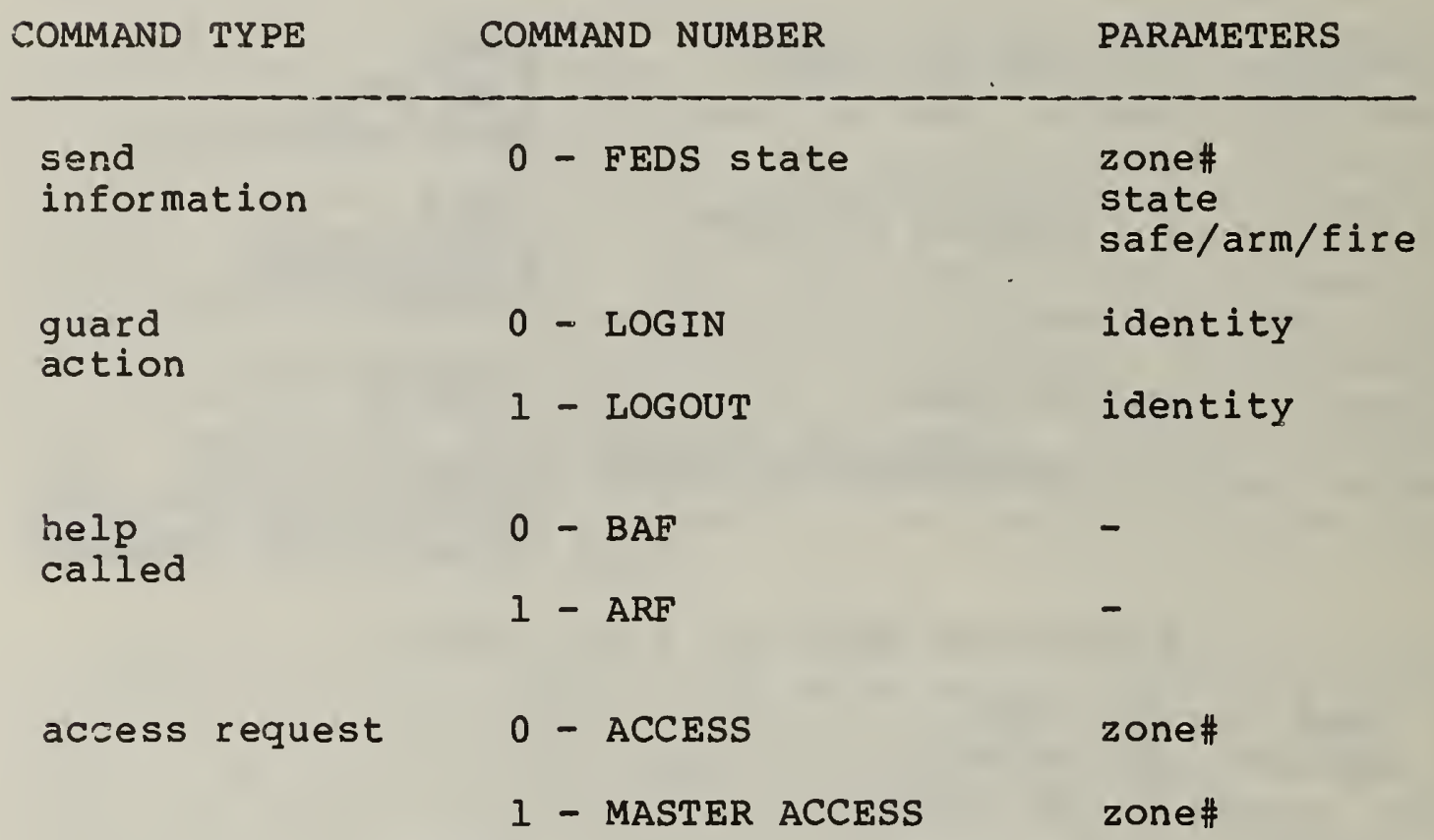

Figure 21. List of messages from Event Process to Director Process.

Director Handler

The Director Handler module processes messages from the Director Process, figure 20. These messages fall into three general categories (1) devices failure, (2) result of pending action, and (3) sensor alarm. 
The first category of Director Process messages, device failure, is concerned with providing the current operability status of the various devices throughtout the entire site (e.g., motion sensor failed at zone 17). This status can be operational, partially operational, and failed. The Event Process updates its data base with this information, and sends both a result message and specific messages to the Console Process.

The second category of Director Process messages, action result, provides the result of a previously requested action that takes place somewhere on the site ( e.g., access successful at zone 10). The Event Process updates its data base, clears the pending status, and sends both a result message and specific messages to the Console Process.

The third category of Director Process messages, sensor alarm, is a spontaneous action that indicates a sensor has changed state, from quiesent to active or active to quiesent. An activated sensor indicates that a disturbance has been detected. This may have been caused by an intruder, or by failure of the sensor. When a sensor is activated the Event Process first tests if that sensor is operational. If the sensor is known to already be in failure mode, the activation is disregarded. The Event Process updates its data base indicating the sensor and zone affected, as well as adding the zone onto the Alarm queue. Also the Console Process is sent a result message causing it to update its local abreviated status and specific messages are sent to inform and evoke a response from the Operator.

Debugger

The Debugger module was used during debugging and is intended for normal preventative maintenance and trouble shooting, similar to that for the Console process. It interacts with a separate terminal, that may be built in or a portable one that can be plugged in when required. It allows maintenance personnel to interactively peruse the data base, status information, and the contents of messages exchanged with both the Console Process and the Director Process, as well as run predefined diagnostics, while the system continues to function. 
The Director Process is the driver for the Event and Console processes. It was designed to accept commands from a human operator via a terminal, translate those commands into one of the messages in figure 20, and send that message to the Event Process. The messages represent the results of actions that may occur at the various zones throughout the site. Since the Event and Console Process require only the resultart action, there was no need to simulate the complex computations required to determine that the actions actually occured.

The Director Process was not designed to keep track of the system state or to automatically execute preprogrammed senarios. Although these features are not difficult to add and they would be useful in a training environment, they were not deemed of high enough priority for our simulation model. The only data base maintained consisted of the current list of active zones and the current list of zones requesting access. Although other information regarding changes in the system status were sent to the Director Process, they were displayed to the Operator and not retained. The Director Process also required the non-blocking read option so that it could constantly poll the terminal for commands from the operator, and the communication link for information on changes in system status from the Event Process.

\section{EXPERIMENTS USING THE SYSTEM}

A series of experiments using the GCS mock-up were conducted. The first of these involved validating the system performance to insure that its behavior was in accordance with specifications in all respects. Next, certain training materials were prepared describing the CSSMRS and the GCS. These were intended to support a tutorial presentation. scenarios were written that could be used to provide handson experience in a simulated physical security environment. These materials were employed in experiments in which coworkers who did not have a background or experience in physical security were trained to use the system. Finally, a group of military personnel were trained to operate the GCS. Observations of these experiments provided insight as to the ease or difficulty of learning the cSSMRS concepts and learning to use the GCS. These observations, together with 
student's comments, also provided the basis for a number of minor changes in the physical arrangement of the console.

\section{Validation of System Performance}

Key aspects of the performance of the GCS in a simulated CSSMRS environment were described by a "partial" state diagram that was amplified by a descriptive event/result sheet that was prepared for each of the active console controls. This process was repeated for each of the system states. The format of the event/result sheets was standardized. On each sheet an event is postulated and the results are described. In most instances, the postulated event is the actuation of one of the console controls. The description of the results includes changes to status as well as changes to any of the displays, messages, audible or visual signals, or other operator cues. This is further expanded by comments where appropriate.

More than 150 of these event/result sheets were prepared using a computer based word processing system. This, together with the standardized format, greatly facilitated making necessary revisions or up-dates. These sheets served as the guiding basis for the simulation software that was developed for the mock-up. While they do not cover every conceivable event that could occur in any possible system state, they are sufficiently broad in scope that the outcome of any event that is not described can be inferred with a high order of confidence.

Validation of the system performance was accomplished by going through each of these event/result sheets and testing to verify that performance was in compliance. This process revealed a number of software "bugs". In most instances they were readily corrected. In a few cases it was determined that it would be better to change the requirements on the sheets. Only a few instances were disclosed where the event/result sheets did not provide complete coverage of the circumstances. Additional sheets were prepared to cover those situations.

The testing and validation process provided an initial indication that the console layout was generally good, it appeared to be easy to use, and was effective in conveying information to the Operator. 


\section{Preparation of Training Materials}

Two types of materials were prepared for use in training personnel to be Operators of the GCS.

The first was an outline of a brief, tutorial instruction course that covered CSSMRS concepts that are essential in order to operate the GCS. These include such things as the subdivision of the site into zones; the association of certain console controls with a zone that has been selected; communication with a zone; access to a zone; alarm acknowledgement and the automatic selection of closed circuit television for assessment; and the use of FEDS and the ARF and BAF response forces. The course also includes a description of the operation of the GCS itself. There are frequent hands-on exercises and demonstrations. The course material can be thoroughly covered in about four hours of time, with additional time required for the hands-on experience if there is more than a single person in the class. The course outline is shown in Appendix I.

Aâditional training materials were prepared in the form of scenarios. These are conducted using an instructor who plays the role of various on-site support personnel such as mobile patrols or maintenance personnel. A second "director" plays the role of adversary by invoking alarms or other events from a nearby computer terminal. The trainee plays the part of GCS Operator and responds to the alarms or other events in what the trainee believes to be an appropriate manner. The instructor and the director have copies of the scenarios to guide their actions, but the student does not. The students actions are guided solely by the various console displays and signals.

When an alarm occurs, CCTV from the alarming zone is automatically selected and displayed on one or both of the previously dark console monitors. In order to provide some basis for reasonable operator responses to simulated alarms, it is necessary to simulate video data from the alarming zone. This was done by placing still, black and white photographs of perimeter fencing in the fields of view of each of three television cameras that were located near the director, figure 18 .

The airector could set appropriate pictures in place before the camera(s), and then type into his terminal the command that would cause an alarm to be reported and simultaneously cause the picture(s) to be displayed on the console monitor. A variety of pictures were available. Some of these showed adversary attacks on the perimeter fence. Others showed an intruder, which in one instance was a deer. In others, there was no visible evidence of what might have caused the 


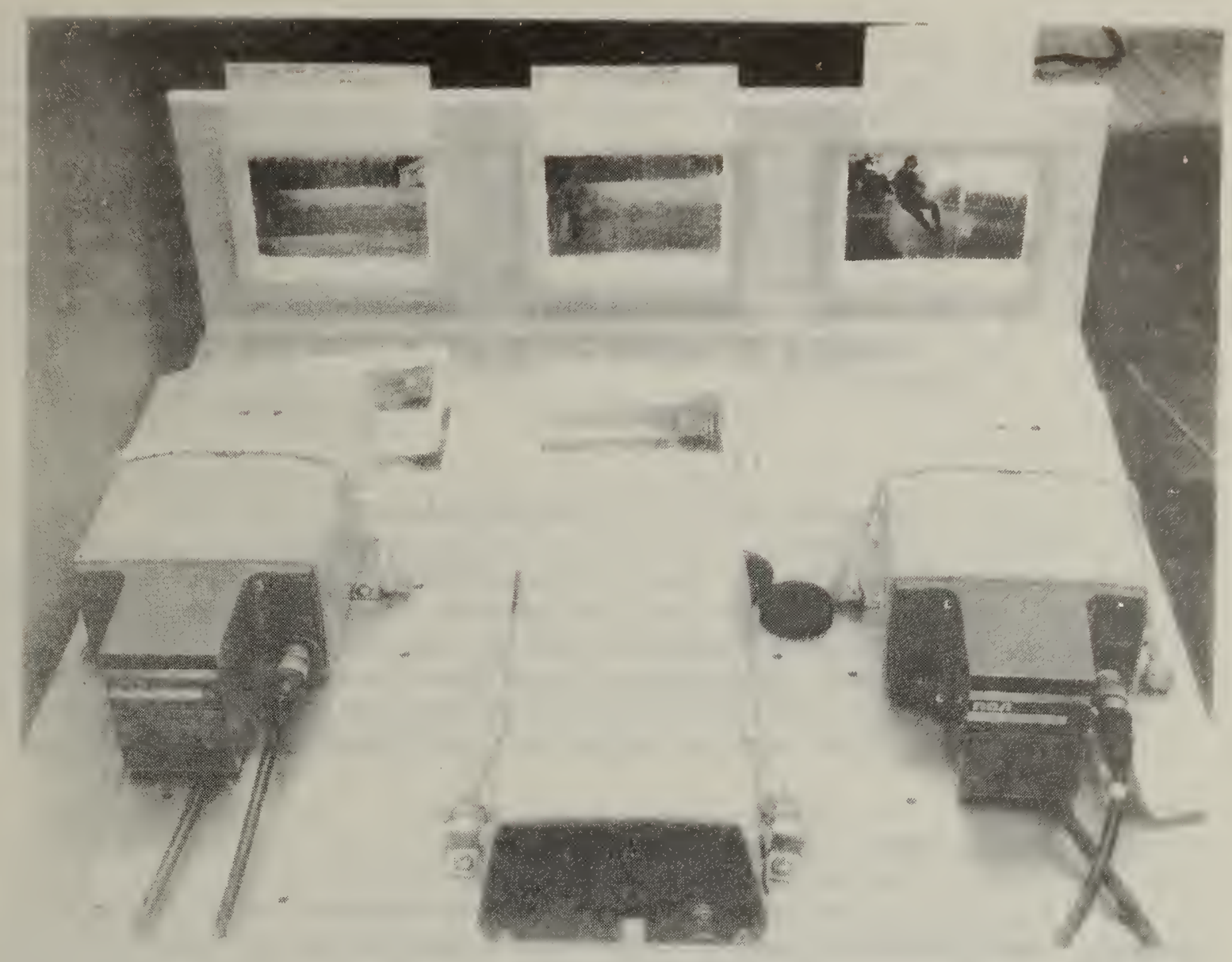

Figure 18. Arrangement of CCTV cameras and still pictures.

alarm condition. One group of these photographs was especially made for the scenarios using a camera and lens combination that would exactly duplicate the fields of view of the two cameras that were specified for use on a 100 meter perimeter segment in [1]. These confirmed that the resolution was adequate to recognize a man-sized intruder anywhere along the full length of the segment.

In addition to the three fixed CCTV cameras, there was a fourth camera mounted in the simulation room that was equipped with controls on pan, tilt, zoom, focus and iris that could be remotely manipulated from the console when the zone with which it was associated had been selected. This was used in conjunction with a group of small targets that had been scattered about the room to give the students an opportunity to develop their skills in camera manipulation. 
The scenarios covered a considerable range in complexity. They ranged from simple exercises intended to develop speed and confidence in finding and operating the various controls to more complex and stressful exercises simulating a multi-person attack on the storage site. They include examples of virtually all of the actions that an operator would be required to participate in, such as key issuance, access, maintenance actions, and testing activities as well as alarm acknowledgement, patrol dispatching and FEDS actuation. An example of one of the more complex scenarios appears in ippendix $I$.

\section{Tests of Training Materials}

Initial tests of the training materials were conducted in two separate sessions using two co-workers who were not Eamiliar with the project or with physical security procedures. In each instance, less than one day was devoted to the training effort. The time was approximately evenly divided between the course material and the scenarios.

Test by an Electronics Technician

The first person to test the training materials was a male electronic technician who had not previously participated in the construction or the console or had any intimate cornection with the project. He was presented with the tutorial course material for approximately an hour, and then was initiated to the scenarios. He quickly learned to use the GCS, but some of his scenario resporse actions were sub-optimal and it was apparant that the time devoted to covering the tutorial material shouid be lengthened. This trainee's handling of the conscie controls was generally good, but on a couple of occasions he made errors in the zone selection process. This served as an alert to a possible repetition of these erzors on the part of future trainees.

Test by a secretary

The second trainee was a female secretary that was reported by her supervisor to be a satisfactory, average performer in her regular job. In this instance, presentation of the tutorial portion of the course material was extended to about two hours before work was started on any of the scenarios. She seemed to grasp the material quite quickly. She had no problems with any of the controls and all of her 
response actions to simulated alarm conditions were judged satisfactory. The only problem that she seemed to experience was that of simulating radio communications with the guard patrols. This was an area that had not been covered in the instructional material, and since she had had no prior experience as a communicator, her difficulty here was quite understandable. In all other respects her performance as an operator of the GCS was highly commendable.

\section{Training of Military Personnel}

During the first week of October, 1982, five male military personnel were trained to use the GCs. One of these was an Air Force Master Sargent with extensive experience in physical security equipment, having served as chief instructor for the Small portable Control and Display system (SPCDS) of the Base and Installation Security system (BISS). The other four were Army Military Police from Ft. Meade with no significant experience with security systems or special weapons storage sites.

Most of the first day was spent going over the tutorial portion of the course material. This was interspersed with frequent hands-on sessions where the students operated the controls and began to get the feel of the system. None of them seemed to have any difficulty understanding the system concepts and learning the meanings of the various signals and displays.

When the tutorial portion of the course material had been covered, all of the students but one were isolated from the simulation laboratory. The one student remaining in that laboratory then operated the GCS while the scenarios were played out. Then another student was brought in to participate in running the scenarios. The reason for the isolation of the others was to keep them all at the same level of training on their initial exposure to the scenarios. Once they had completed the scenarios they were permitted to remain in the simulation laboratory to watch the next student's actions. All of them performed quite well, and very few mistakes were made. Those few generally involved zone selection, but the trainees themselves did not seem to notice this. Occasionaly a student would make a response that, while not exactly in accordance with the response anticipated in the scenarios, was still acceptable. In these cases the instructor and director were nearly always able to make impromptu moves that would lead the simulated action back to the prepared script. 
When all of the trainees had completed all of the prepared scenarios, they were given brief instructions in the operation of the computer terminal used by the director to simulate adversary actions. They very quickly mastered this, and then proceded to generate and act out impromptu scenarios, with each student taking turns at playing the different roles of director, instructor, and operator. They became quite proficient at this, and generated a number of very realistic event sequences. They became adept at keeping track of the locations of the simulated patrols that they dispatched to assess alarm conditions or conduct maintenance activities. They conducted impressive demonstrations for several visitors to the simulation laboratory, and seemed to be thoroughly comfortable and at home with the system.

At the conclusion of the training sessions, they were each given a questionaire to complete. This was intended to draw out their comments about what they found to be good or bad about the arrangement of the console and its controls and cisplays. A copy of the questionaire and their combined responses and comments appears in Appendix II.

\section{SUMMARY AND CONCLUSIONS}

Observations of the performance of the seven individuals that have been trained to operate the GCS provides convincing evidence that most of the original design objectives have been met. The trainees invariably found that the GCS was easy to learn and easy to use. They did not find it intimidating or overpowering and seemed to feel quite comfortable with it after a relatively short period of time. The displays, cues and guidance messages were judged adequate in terms cf size, resolution and information content, but flither improvements can be made by relocating certain displays and controls, and by adding another group of displays. The queueing of multiple alarms, and the operator controlled display of detailed information about only one alarn at a time provided obvious relief from information overload. This was in marked contrast to the more congested display of status information that appears in chronological sequence on the DDU when the zone that has been selected is secure. 


\section{Console Design Changes}

Based on a review and analysis of both our observations and the comments of the trainees, a number of changes to the design of the GCS console are being reflected in the specifications for a prototype CSSMRS. These are discussed in detail in the following sections.

\section{0-Digit Keypad}

On the mock-up, a 10-digit keypad is located on the right hand side of the console table. This is used to enter digital information into the system. The digital information is either a 10-digit operator identification number or a one-to-three-digit zone number. The number that is entered on the keypad appears on a 10-digit liquid crystal display directly above the keypad. When a zone number, consisting of only one-to-three-digits, is entered, nothing else happens until the SELECT ZONE control is operated. At that time, the number that has been entered in the 10-digit display is transferred to the 3-digit ZONE SELECTED displays, and the 10-digit display is cleared. Console controls and CCTV (if available) are attached to the zone that has been selected.

On certain occasions, student GCS operators were observed to be watching the ZONE SELECTED display rather than the 10-digit display as they made entries on the keypad. There was hesitancy and a brief period of confusion before they realized what the problem was. Additionaly, observations of hand movements indicates that a faster, more positive zone selection would occur if the 10-digit keypad is relocated to a position directly below that presently occupied by the ZONE SELECTED display on the console table. The SELECT ZONE control will be moved from its present location and will appear as a pair of keys, one located on either side of the keypad.This will permit zone selection to be made by operation of either key, using either the thumb or little finger. The entire process can be completed without the arm movement that is presently required on the mock-up.

The relocated keypad will be used only for entering zone identity and not for entering operator identity. The keypad will require much better tactile characteristics than the keypad used on the mock-up. The feel should be similar to that of the keys on the typical computer terminal or electric typewriter. In addition, the keys should be color coded with the electronic industry code as well being labeled with the digit value. This will permit the zones in small sites to be identified by "color" rather than "number" 
if desired. A software switch should be provided so that zones zero through nine can be optionally identified by a color instead of a number. The equivalence of number, color, and a three character abbreviation of that color are shown in Table 8 below.

TABLE 8

Color Code For zones 0 - 9

$\begin{array}{clc}\text { Zone } & \text { Color } & \text { Abbreviation } \\ 0 & \text { Black } & \text { BLK } \\ 1 & \text { Brown } & \text { BRN } \\ 2 & \text { Red } & \text { RED } \\ 3 & \text { Orange } & \text { ORN } \\ 4 & \text { Yellow } & \text { YEL } \\ 5 & \text { Green } & \text { GRN } \\ 6 & \text { Blue } & \text { BLU } \\ 7 & \text { Violet } & \text { VIO } \\ 8 & \text { Gray } & \text { GRA } \\ 9 & \text { White } & \text { WHI }\end{array}$

The three-digit ZONE SELECTED display will be replaced with a three-character display labeled ENTRY. Keypad entries will appear here until the SELECT ZONE (or CLEAR) key is depressed, and then this display will go blank again. When the software switch is set to COLOR, the display will show the three character abbreviation of the color of the key that is operated. When the switch is set to NUMBER the display will show the numerical value of the keys (up to three digits) that are actuated. When the SELECT ZCNE key is actuated, the information shown in the display is transferred to the other ZONE SELECTED displays.

\section{Selected Zone Status Display}

The six colored lamps at the top of the console panel provide a "quick look" indication of overall site status that is more useful to a supervisor than to the Operator. As long as the Operator has selected a zone that is in an alarmed state his display provides him with complete and uncluttered information about that zone. However, when the Operator selects a zone that is not in the alarmed state, the display shows status information that may involve a number of zones. The Operator must read through this to learn the status, such as ACCESS, REPAIRS REQUIRED, or other conditions affecting a particular zone. 
Two changes will improve this situation. The information on the status display can be better organized (this will be described in a following section), and a zone status display can be added. The added zone status display will contain a three-character LCD display and six colored lamps which replicate all of the functions of the site status summary group. This zone status display will be mounted on a panel located directly behind the MPS and beneath the DDU. When an operator selects a zone, either by use of the keypad or by operation of the MPS to select an alarmed zone, the identity of the selected zone will appear in the threecharacter display. Zone identity may be either a number or color depending on the setting of the software switch. The status of this zone will be indicated by illuminating one or more of the six associated status lamps.

\section{Organization of Status Display on DDU}

In the mock-up, status information is displayed on the DDU whenever the selected zone is in a non-alarmed state. The information is ordered chronologically rather than being grouped by class or condition. Thus, if the white, REPAIRS REQUIRED, summary status lamp is lit, it may be necessary to scan the full list of status messages in order to ascertain which zone(s) require maintenance. This will be changed, and the entries will be grouped by class, and listed chronologically within each class. Alternate classes will be in reverse video to define the class without loss of screen area that would be required to provide separation by inserting a blank line between groups, however, if any class is empty, a blank line will be inserted in its place.

The top line of the status display will be used for reporting the number of outstanding uncleared alarms. This report will be in double width letters, and will blink continuously so long as there are any outstanding alarms. Video will not be reversed.

The first group will be reverse video and display any zones in Access.

The second group will be normal video. It will display any time dependent actions such as KEY SENT. The remaining time on any items in this group will be continuously decremented at intervals of not less than one minute, and the entry will be removed when the time limit has expired. 
The third group will be reverse video. The entries will be updated with each automatic cycle of sensor and FEDS tests conducted by the central processor unit and any failures are listed. The entries will also list all other maintenance actions that are outstanding.

The fourth group will list the names, but not identity numbers, of operators logged in and on duty, in normal video.

A variable size window at the bottom of the screen will be used to display operator guidance messages as on the mock-up. It and the top line, displaying uncleared alarms, will not be affected by the scroll control, while the other four groups of status messages will be scrollable.

\section{Log in/Logout}

The provisions for operators to login and logout of the system will be revised to protect the identification number of operators from being disclosed to possible unauthorized or unqualified personnel. This will be accomplished by removing the present 10-digit keypad, 10-digit display and the LOGIN and LOGOUT controls from the console table and locating them in a recessed, covered enclosure which may be at any convenient location in, or adjacent to the control room containing the GCS. Again, the keypad will have greatly improved tactile characteristics as compared with the one used in the mock-up, but color coding is not required here.

When a valid login occurs, the operators name but not identity number will be displayed on the status display. The voice annunciation will be truncated to, "Valid (Invalid) Operator Login (Logout)", since it would be impractical to continuously update roster names for the voice unit.

\section{CCTV Facilities}

On the mock-up, there is a three-digit display indicating the selected zone and a cluster of lamps indicating TV facilites at that zone associated with each of the monitor pairs. They are located to the left of the left hand monitor pair and to the right of the right hand monitor pair. These were judged to be too far toward the periphery of vision, and will be moved to a position between each of the monitor pairs and as close to the DDU as feasible. The three-digit displays will be changed to three character displays to accomodate zones identified by colors, and an 
up-arrow and down-arrow will be added to each. These arrows will be selectively illuminated to point to the monitors that should be active for the selected zone. They will provide cues to CCTV malfunction, since if both arrows are illuminated and only one monitor is operating, there is evidence of a problem with the dark video channel.

Increased visibility of the CCTV facilities indicators will be provided by removing the smoke colored indicator covers and using covers with greater translucence or less attenuation. The intensity of the lamps in all of the indicators has been lowered somewhat by operating them at a reduced voltage ( 5.0 volts rather than 6.3 volts) in order to provide a projected life of 290,000 hours ( 33 years). This is in keeping with overall CSSMRS reliability objectives.

Pan/Tilt/Zoom Control

The Pan/Tilt/Zoom CCTV control will be moved back about $10 \mathrm{~cm}$. (three inches) farther from the edge of the console table. This location is judged to be more appropriate to the expected frequency of use since few zones are expected to be equipped with cameras that are controllable. The new location will improve the access and visibility of the controls associated with the electronic key.

Monitor Pointing Switch

The handle on the MPS will be shortened by about three cm., and the throw of the switch will be increased to a full 90 degrees, side to side. This control is the single, most used control on the console, and it's continued operation is vital to the operation of the system. Further reductions in size would be at an unacceptable cost of reduced mechanical strength and durability.

Scroll Control

The SCROLL control will be moved to a position to the right of the MPS and symmetrically located with the SAFE FEDS control. 
Emergency Controls

The CALL BAF and the CALL BAF \& ARF controls will be separated from each other and placed in locations that are symmetrically aligned with ARM FEDS and SAFE FEDS. A locked, key operated switch labeled FIRE WADS (weapons Access Denial system) will be located in line with these two controls and aligned with FIRE FEDS.

\section{Key port}

The system specifications will make provision for a second key port that duplicates the functions of the console key port but is remotely located from the console. The location of this duplicate key por: may be outside of the control room so that it is possible to load an electronic key without having to gain access to the control room.

\section{Electronic Key}

The final design of the electronic key will incorporate an LCD display that shows the remaining key life. This display will be in minutes and seconds, and will be updated each second.

Writing Shelves

A pair of pull-out shelves will be provided on the console, one on either side of the operator. These can be used as surfaces for writing.

\section{Summary of Changes}

Figures 19 and 20 show the general arrangement of the GCS console after these changes have been reflected. Figure 19 shows the console back panel and figure 20 shows the console table top. 


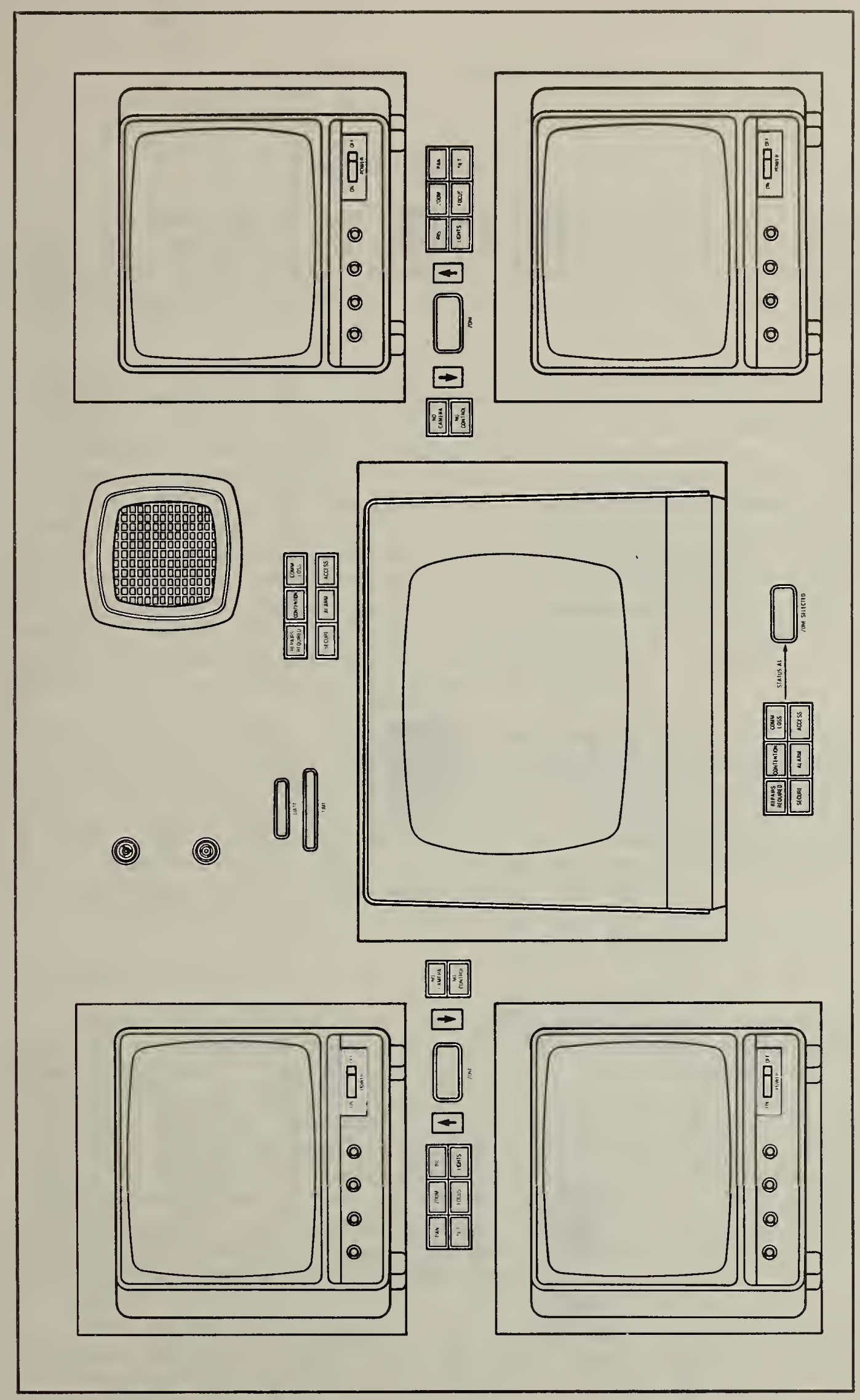

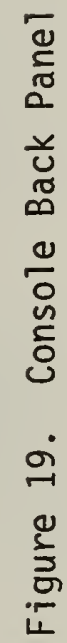




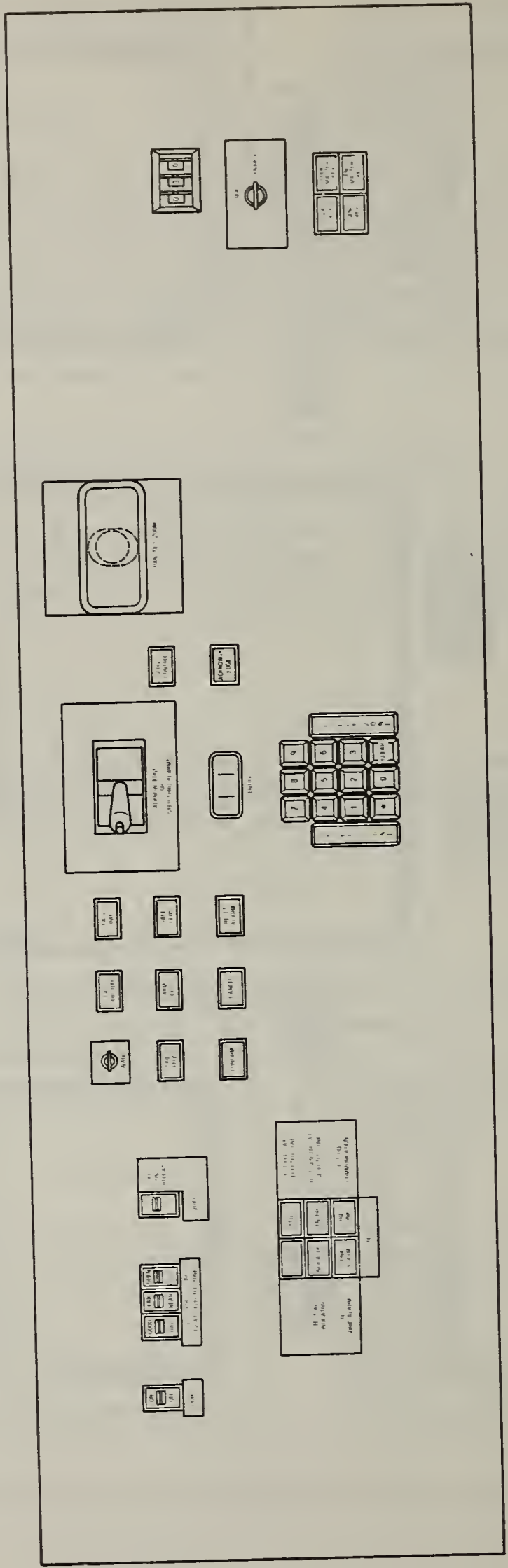

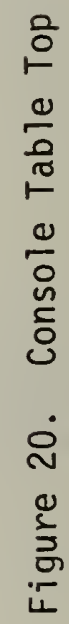




\section{Conclusions}

The mock-up of the GCS and its operation in a simulated physical security environment has provided an excellent opportunity to evaluate both the ergonomics of its design and the potential utility of such a simulator as a training device.

Observations of the actions and performance of trainees, and careful evaluation of their comments in relations to these observations has suggested a number of minor changes in the arrangement of console controls and indicators. Their inclusion in the design of a future prototype model of the CSSMRS will make small, but useful improvements in the speed, accuracy, and ease of GCS operation. It is believed that none of these changes will have noticeable effects on the process of learning to use the GCS, as it is felt that the principal objectives with respect to this process were attained with the original mock-up design. None of the seven students that were trained appeared to experience any difficulty in learning to operate the GCS.

As a training tool, the present implementation of the system has several significant, but potentially correctable deficiencies. These involve characteristics of the programming that was prepared for the director processor and its terminal, the CCTV video presentation to the Operator, and the fact that several important adjuncts to the CSSMRS GCS were not implemented in this mock-up.

\section{Director Scftware}

The software for the director processor was not arranged to support several capabilities that would be highly desireable on a training simulator. For example, there was no provision by which multiple alarms could be simultaneously introduced at several zones, or by which alarms at several zones could be quieted simultaneously. Alarms at each $z$ one had to be introduced and quieted individually by keyboard entries at the director's terminal. The delays associated with this process would prevent a realistic simulation of certain classes of events, and errors in kejboard entries can cause unintended results.

For training purposes, it would be highly desireable to program entire scenarios so that they could be completely executed by the director processor without a requirement for interaction by anyone other than the student trainee at the GCS. The processor could even be programmed to score the trainee's performance. This would reduce the manpower 
required to conduct a training session.

GCS Video

The use of black and white, still photographs as a demonstration of the resolving power that results from the use of the recommended arrangement of perimeter CCTV cameras proved to be quite effective. Those same photographs, however, are not very realistic alarm assessment imagery. The lack of motion, and the small number of cameras that made it necessary to reuse a given camera on more than one zone, all detracted from the illusion of reality. We would recommend that a training facility be equipped with from eight to twelve video tape units. There should be one for each zone of the simulated facility that is equipped with CCTV. Their tapes should be pre-recorded with scenes and actions that are appropriate to the director processor's scenario, and they should be started simultaneously at the beginning of the scenario, and should run continuously for its duration. This could provide extremely realistic video. For example, if a student should happen to be attached to a zone where the scenario had scheduled an alarm to occur, the student might actually see the intruder approach the perimeter a second or so before the alarm was triggered!

Features Not Implemented

We recommend that a simulation training facility should be provided with several of the GCS adjunct facilities that were not implemented on this mock-up. These include a Geographical Display Unit (GDU) replicating the layout of the simulated site and equipped with status indicator lamps for each zone. This is particularly important as the console DDU does not present any geographic information to the operator; it was intended that this would be provided by the GDU. It is needed in order to provide the operator with an overall perspective on the locations of possibly related alarms.

Another facility that is not adequately implemented on the mock-up is that for communications, including radio, telephone and intercomm. In fact, until these have been implemented and tested, it is not clear whether or not a single operator can handle the work load, or whether some portions of the communications functions may have to be handled by another person. 
The logging functions should also be implemented in a facility intended for training in order to insure that students are properly instructed in the procedures for recording the dispositions of alarms. This administrative function was not addressed at all in the exercises involving the mock-up.

With the implemetation of these, additional adjunct facilities and capabilities, we believe that a highly effective training facility could be set up based on the general software approach to simulation that has been demonstrated in this project.

\section{BIBLIOGRAPHY AND REFERENCES}

1. R. T. Moore, R. J. Carpenter, A. W. Holt, A. L. Koenig and R. B. J. Warnar, "Phase II Final Report Computerized Site Security Monitor and Response System", National Bureau of Standards, NBSIR 79-1725, PB 294343 , NTIS, springfield, VA 22161.

2. Military Standard, Human Engineering Design Criteria for Military Systems, Equipment and Facilities, MIL-STD-1472A, Department of Defense, Washington, DC 20301 .

3. Kernigham, B. W. and Ritchie, D. M., "The C Programming Language", Prentice-Hall, Inc., Englewood Cliffs, NJ, 1978 .

4. Ritchie, D. M., and Thompson, K., "The UNIX Timesharing System", CACM, Vol. 17, No. 7, July 1974 . 


\title{
GLOSSARY OF ACRONYMS AND ABBREVIATIONS
}

\author{
ACK Ack nowledge \\ ARF Augmentation force. This force may be located at \\ higher headquarters. \\ BAF Back-up force. This force is located on or near \\ the secure site. \\ CCTy closed circuit television. \\ CSSMRS Computerized Site Security Monitor and Response \\ System. \\ FEDS Forced Entry Deterrent Systems. \\ GCS Guard Control station. \\ HC Headquarters. \\ MPS Monitor Pointing Switch.
}




\section{APPENDIX 1}

Outline of GCS Console Instruction Course

\section{Background of CSSMRS}

A. Experiment to see if modern technology can be used to improve the physical security of special Weapons storage sites.

B. Use the computer to help make the guard's job easier. Provide guidance toward the right choice of action.

C. Does not require that the guard know anything about computers or know how to type. The system uses functional controls with guidance by means of lights and voice announcements as well as printed messages on the screen.

D. A novice cannot do anything to hurt the system. If the wrong control is pushed, it will tell you, or ask you to Cancel or Confirm that order. In other words, "Do you really want to do that?"

E. Our objective is to find out whether or not it really is easy to learn to use, and if it is not, find out what to change before a prototype is built.

\section{CSSMRS Concepts}

A. The site is divided into zones - each zone is $100 \mathrm{~m}$. perimeter - one football field long - or one magazine or bunker

B. Each zone has the following:

Intrusion sensors which might include:

$\begin{array}{ll}\text { Maid/Miles } & \text { Radac } \\ \text { Motion (ultrasonic, RF or TV) } & \text { Spir } \\ \text { E-Fild } & \text { Blid } \\ \text { Vibration (three types) } & \text { Optical } \\ \text { Magnetic Switch } & \text { Infra-red }\end{array}$


Explain each

Closed Circuit Television to assess alarms -

TV may include controls and lights

Forced Entrance Deterrence Systems

Gas

Smoke

Pyro

Explain each - all are conceptual

A Computer

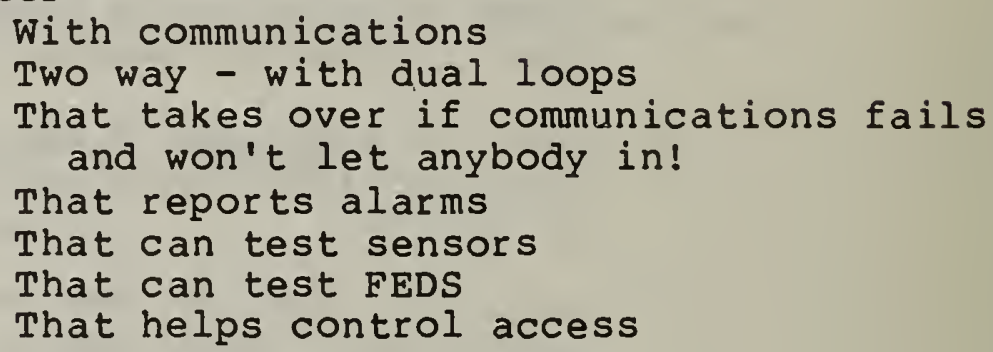

C. There is a Central station with computers

It acts as a link between the zones and two GCS

It talks to the zone computers

It runs the controls and displays on the GCS

It talks to higher headquarters

It calls BAF or BAF and ARF if the GCS operator is disabled

D. There are two Guard Control stations in a real CSSMRS (only one has been implemented in this model) which:

Permit interactions between the guards and the system by:

Displays

$$
\begin{aligned}
& \text { Hand out list } \\
& \text { Identify each on GCS }
\end{aligned}
$$

Signals and voice announcements

Controls

$$
\begin{gathered}
\text { System - hand out list } \\
\text { Identify each on GCS } \\
\text { Attachable - hand out list } \\
\text { Identify each on GCS }
\end{gathered}
$$

Demonstrate simple interactions - provide hands-on practice: 


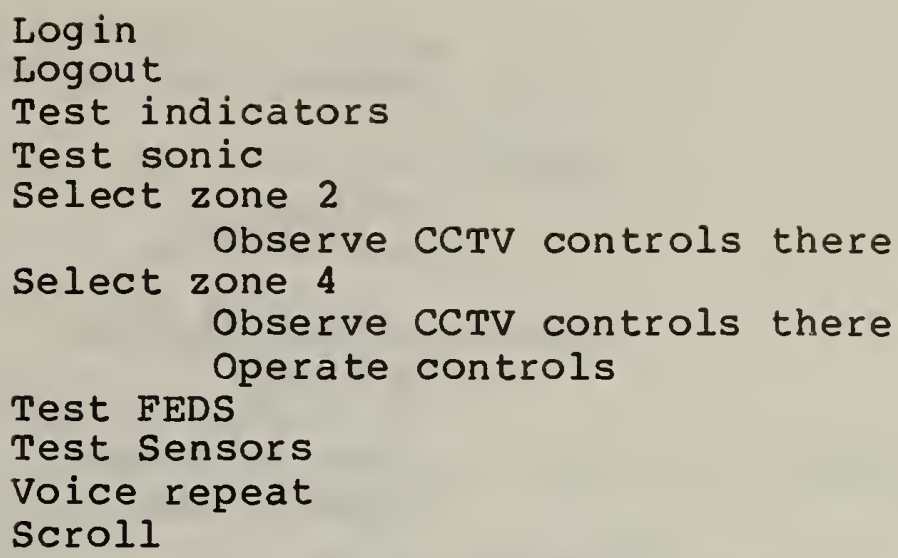

E. Each zone has security status that depends on prevailing conditions

Hand out list of status displays

SECURE

Define

status information on display

Most likely controls to use are lighted

CCTV (if any) on monitor that MPS is pointing toward

Demonstrate above

ALARM UNACKNOWLEDGED

Define

Voice and display announcement

Audible alarm sequence begins

Flashing lights in controls

Most other controls disabled

CCTV from new alarm on the dark monitors

MPS not pointing to these

BAF call out if no acknowledgement

Demonstrate above

ALARM ACKNOWLEDGED

Define

Display shows full alarm report

Audible alarm sequence is stopped

status light is on steady

Most likely candidate controls are lighted

Controls associated with zone MPS pointing toward

Demonstrate above

ACCESS

Define

Most likely candidate controls are lighted 
Status information on display

Demonstrate above

\author{
REPAIRS REQUIRED \\ Define \\ Demonstrate
}

COMMUNICATIONS FAILURE

Define

No controls operable at this zone

Zone autonomous

Demonstrate

CONTENTION

Define

Not implemented in this model

3. Instruction in the use of Guard Control station

Review use of keypad and significance of lights

A. When an alarm occurs

TV from the alarming zone appears on the previoulsy

blank screens

Appears immediately

Provide opportunity to catch moving targets

Alarm must be acknowledged to prove to the system

that the guard is not disabled.

30 seconds - one tone

30 more seconds - another tone

10 more seconds - a buzzer

Then BAF is automatically called

How would you like to face your buddies if you dragged them out of the sack for not acknowledging an alarm on time?

Two ways to acknowledge an alarm

MPS - attaches controls to alarmed zone

ACKNOWKEDGE - does not attach controls to zone

Demonstrate each way

After acknowledgement, must find out what caused alarm

Examine scene in CCTV

Send patrol to investigate

If intruder is observed, there are options

send patrol to apprehend

Actuate FEDS

Demonstrate simulated radio call

Demonstrate 


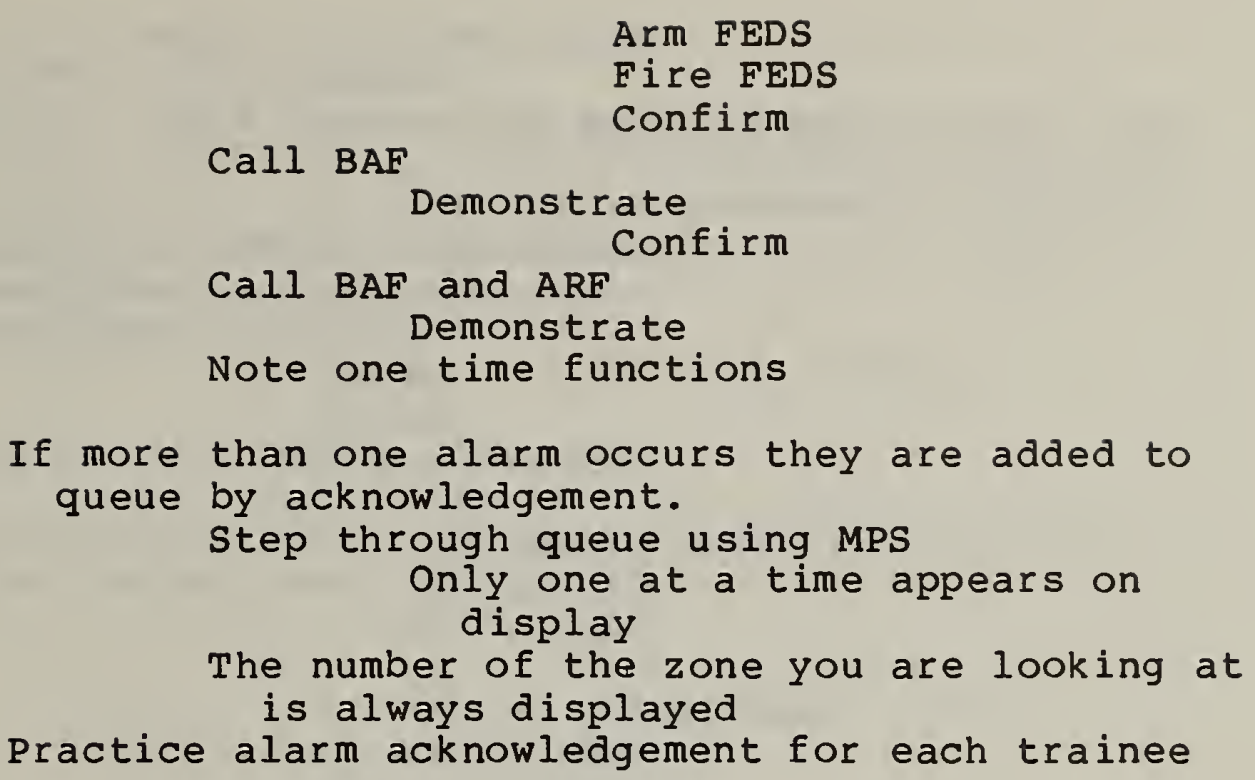

B. Clearing alarms

Conditions for clearing:

You must be satisfied that there is no threat

The condition causing the alarm must have gone away

Sensors at the zone must be working Test Sensors

Clear by operating RESET ALARMS

Alarm message replaced by status message Control lighting changes

Practice clearing alarms for each individual

C. Access Procedures

Purposes of access

Move SNM

Repair or maintenance

Authorizations

Two man rule

Mechanical and electronic keys

Coding Key

Lock/Enable

Key life - Two digit thumbswitch

One-time key

Only one outstanding 


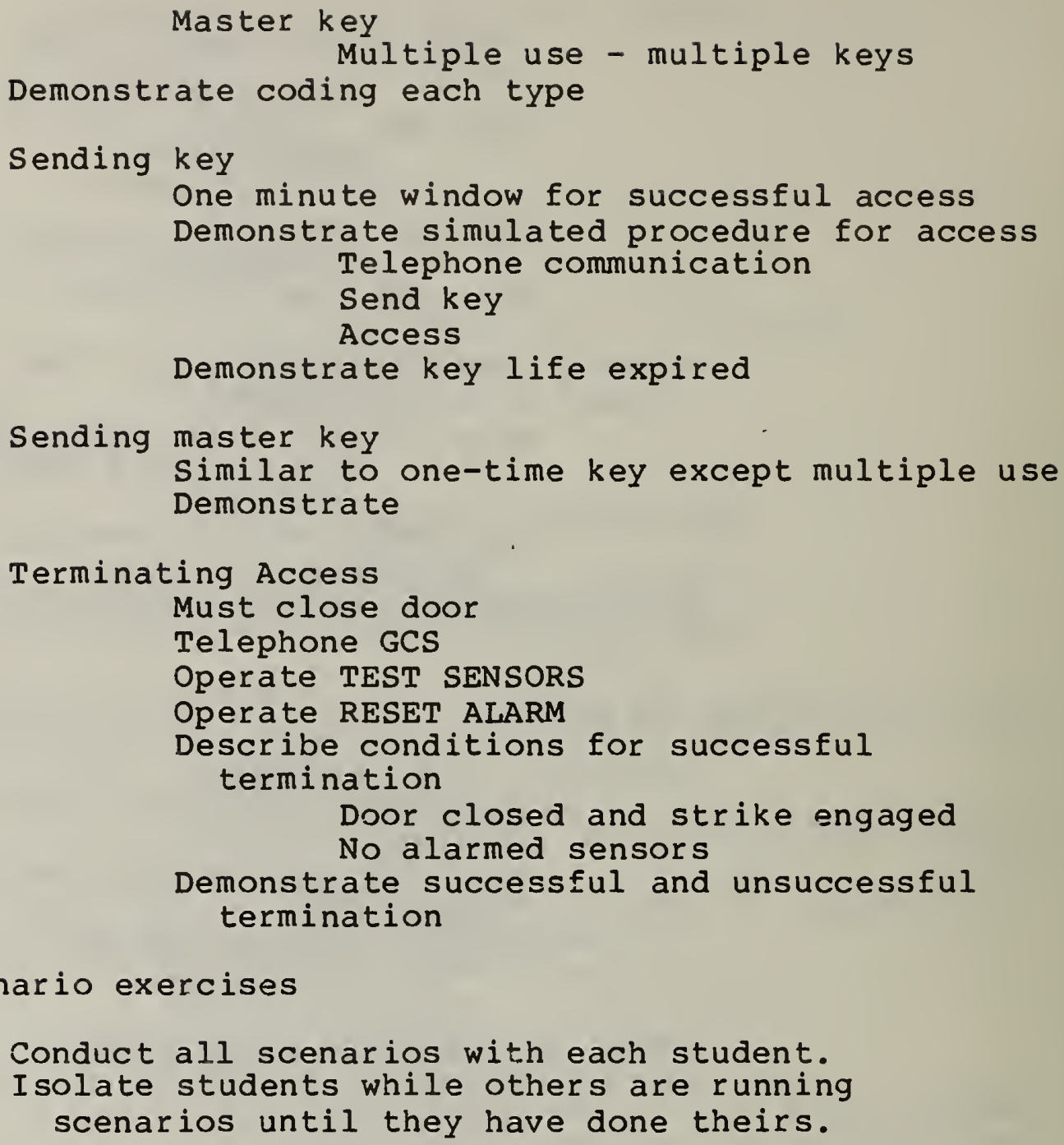

Conduct all scenarios with each student. Isolate students while others are running scenarios until they have done theirs. 
A Sample Scenario

The simulated site consists of ten perimeter zones that are numbered one through ten, and nine magazines that are numbered 11 through 19. In addition, the entrance portal is designated zone 116, and the Site security Control Center is designated zone 114 .

SITE LAYOUT FOR SCENARIO SHOWING ZONE NUMBERS

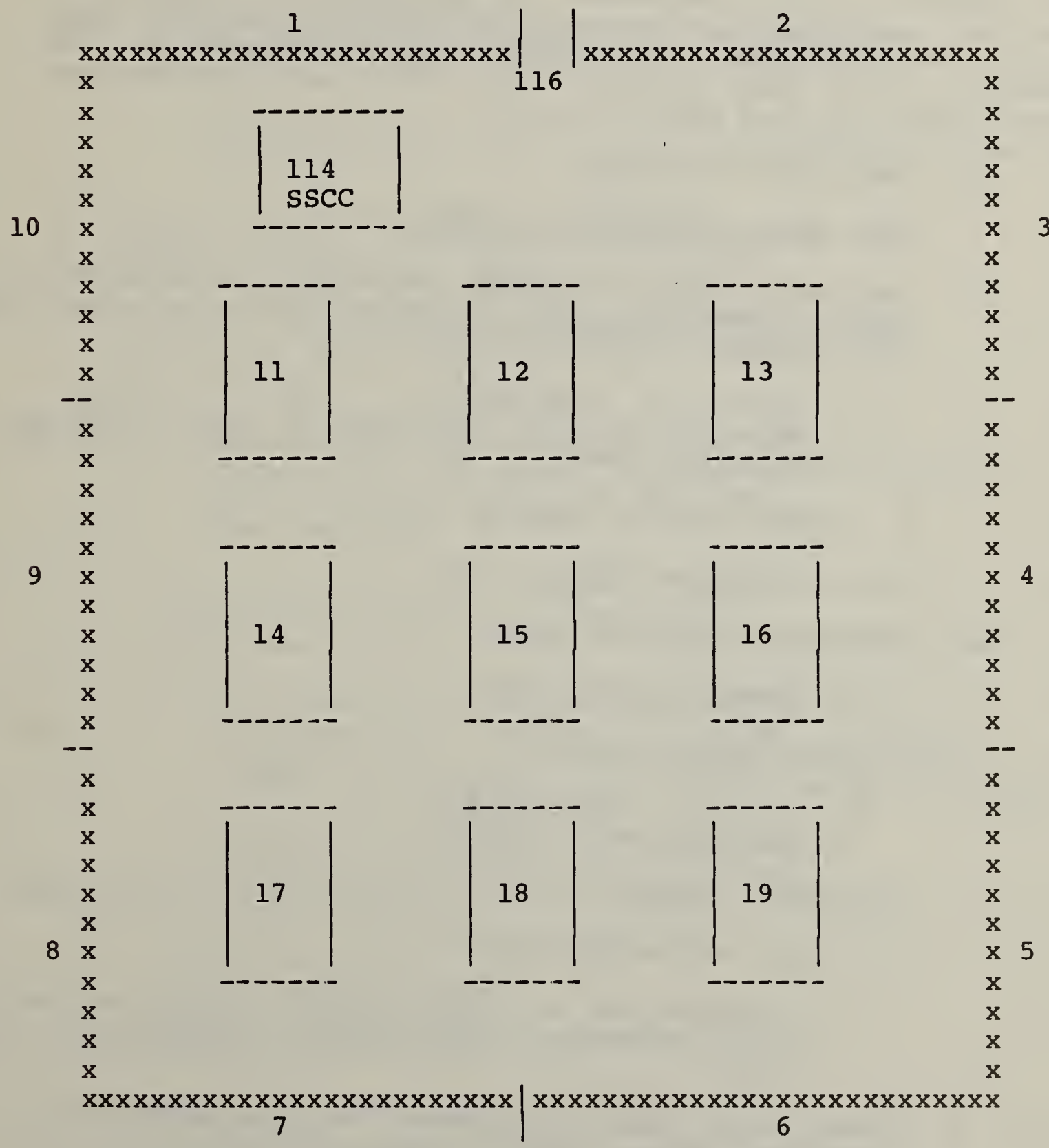


INITIAL CONDITIONS:

The site is secure, but zone 15 is operating on backup battery power. An instructor, (I), is logged in on the system and can simulate the activities of a second GCS operator (0).

(I) will also simulate the actions of maintenance personnel and of patrol ABLE and BAKER.

The director of the simulation (D) operates the "Terrorist" terminal and mounts the photographs for the fixed CCTV cameras. The instructor, (I), observes the performance of the operator, (0), and records any errors and any instances where 0 did not know what to do next.

1. I: Requests 0 to login.

2. O: Enters 1234567890 , LOGIN

3. I: Announces that maintenance personnel are going to $f i x$ zone 15, and that they should be given a 10 minute key. "Let me have a key for zone 15".

4. 0 : Enters 15, ZONE SELECT

Puts key in port and enters 10 (min), CODE KEY Gives key to I.

5. D: Issues test HQ command

5. O: Operates TEST HQ LINK

7. I: Requests 0 to test lamps

8. 0 : Operates TEST LAMPS

9. D: test sensors zone 2

10. 0 : Enters 2, ZONE SELECT Operates TEST SENSORS

11. I: Positions himself at door of room to act as intruder

12. D: alarm motion zone 116

13. O: Operates MPS and CCTV CONTROLS to assess alarm, and dispatches Patrol ABLE to investigate at zone 116.

14. I: Using local phone, announces: "We, the maintenance personnel, are at zone 15 and want access." 
I moves out of the doorway and is no longer visible in the CCTV monitor.

15.

D: quiet motion zone 116

16 .

O: Enters 15, ZONE SELECT

Operates SEND KEY

"Go on in"

17. D: open door zone 15

alarm e-field zone 3

after instaling perimeter zone photographs

$A 5$ and $B 5$ in cameras $A$ and $B$.

18. 0 : Operates MPS

and dispatches patrol BAKER to investigate zone 3

19. I: Simulating patrol ABLE at zone 116, advises 0 that an intruder has been apprehended at zone 116 and that the area is clear.

20 I: Unless 0 does so without prompting, he should be asked to try to secure the alarm at zone 116 . Note if prompt is required.

21.

0 : Operates MPS to bring up zone 116

(Enter 116, ZONE SELECT is an acceptable but less efficient series of actions.)

Operates RESET.

22. D: As soon as operator releases zone 3, install pictures $A 6$ and $B 6$ and generate $a$

alarm maid zone 3 as soon as zone 116 is secure.

23.

0 : Operates MPS to acknowledge alarm at zone 3. (He should now recognize intruder is in the middle of the clear zone between fences and should advise the enroute patrol BAKER of this).

24.

O: Simulates radio call to patrol BAKER

"There is one man inside the fence."

25. D: Disables CCTV covering zone 3 quiet maid zone 3 quiet e-field zone 3 close door zone 15 good power zone 15

26. O: Operates TV BAD control.

27. I: Simulates telephone call from maintenance 
personnel at zone 15 indicating that they have vacated and locked the zone and that it may now be secured.

28 .

O: Enters 15, and RESET.

(O should also advise the maintenance personnel

of the $\mathrm{TV}$ failure at zone 3 , and instruct them

to return for an electronic key to that zone.

28. D: alarm motion zone 18

29. O: Operates MPS to acknowledge.

Finds there is no CCTV to assess, so instructs patrol ABLE to investigate.

30. D: quiet motion zone 18

bad motion zone 18

31 I: Simulates radio transmission, "This is patrol BAKER. There is no one in zone 3."

32 .

O: Reply, "OK, resume regular patrol."

Operates MPS to bring up zone 3

Operates RESET.

33. I: Simulates radio transmission, "This is patrol ABLE. The door at zone 18 is locked and all appears secure"

34 .

O: Reply, "OK let me try to secure the zone" Operates MPS to bring up zone 18 .

Operates RESET. (It fails)

Operates TEST SENSORS. (They fail, white light on)

35 .

O: Radio transmission to patrol ABLE, "The sensors have failed test at zone 18. Post a guard there until we can get them fixed."

36. I: "OK, I'm leaving Smith on guard at zone 18".

37. O: Operates RESET. (Green light comes on)

38. I: Simulates maintenance, "We are here, what is the problem". (Preferably o should send them to fix zone 18 and relieve the guard ahead of making the CCTV repair at zone 3 )

39. 0 : Enters 18, ZONE SELECT

Puts key in port, enters 10 MINS., CODE KEY Gives key to I.

40. D: Issues command to test sensors zone 11 
41. 0 : Enters 11, ZONE SELECT

Operates TEST SENSORS

42. I: "We are ready to access zone 18"

43. $0:$ Enters 18, ZONE SELECT

Operates SEND KEY

"Go on in"

44. D: open door zone 18

45. D: Inserts picture with deer in camera $C$ and "alarm e-field zone 6".

46.

$0:$ Operates MPS to bring up zone 6 .

Sees deer and radios patrol Baker,

"There is a deer in zone 6. See if you can chase him over the fence."

47. D: good motion zone 18

48. I: "I think we have cleared the problem here at zone 18. Will you please test sensors"

49 .

0: Enters 18, ZONE SELECT

Operates TEST SENSORS

Responds, "Sensors test OK now. You can close up and bring Smith back with you. Remember, I've got another problem at zone 3."

PAUSE

50. D: close door zone 18

51. I: "We have locked up zone 18 now"

52. 0 : Operates RESET

53. D: Puts deer picture in camera $A$ alarm maid zone 7

54. 0 : Operates MPS and again sees deer

Radio to patrol BAKER, "Now he is in zone 7."

55. D: test sensors zone 12

$56 \quad 0$ : enters 12 , SELECT ZONE, TEST SENSORS

57. D: Replaces deer picture with clear zone camera A

58. I: "This is BAKER. We see him. He just jumped the 
outer fence."

59. D: quiet e-field zone 6 quiet maid zone 7

60. O: "Good, I'll secure both zones" Operates MPS

Operates RESET

Operates MPS

Operates RESET

61. I: Advises 0 that maintenance personnel are back and want key for zone 3 to fix the CCTV problem there.

62. 0 : Enters 3, ZONE SELECT

Puts key in port, enters 10 (min.), CODE KEY Gives key to I

63. D: test sensors zone 4

64. O: Enters 4, ZONE SELECT

Operates TEST SENSORS

(Sensors test OK, but note that there is still no CCTV working at zone 3 ).

65. I: Simulating maintenance, "We are ready to access zone 3 now and fix your CCTV".

66. O: Operates SEND KEY

"Go on in"

67 .

D: Permits successful access

Requests TEST SENSORS ZONE 4

(As soon as 0 releases zone 3 , restores the CCTV cameras A \& B, pictures A9 \& B9).

68.

O: Operates 4, ZONE SELECT

Operates TEST SENSORS

69. I: Simulating maintenance, "I think we have

fixed your problem at zone 3. Check your TV"

70. 0 : Operates 3, ZONE SELECT

(Finds both monitors working)

Operates TV OK

Advises maintenance personnel that it is OK.

PAUSE 
71. D: close door zone 3

72. I: "We have closed and locked zone 3"

73. 0 : Operates RESET

74. I: Would you like to log out now?

75. O: Enters 1234567890 , LOGOUT

(Another guard will have to login before this logout is accepted by the system). 
APPENDIX II

Summary of Responses to Request for Comments

The following questionaire was given to each of the military personnel who participated in the training program. Their responses have been assembled (exactly as received) and associated with a letter identifier that protects their privacy.

\section{COMMENTS}

\section{Oc tober 7,1982}

In addition to any other comments that you may have about the Computerized Site Security Monitor and Response system concept and the simulated implementation of it that you have been working with for the past three days, please address the following questions about the console itself. Your thoughtful responses, together with the rationale for them, will be greatly appreciated and will be most helpful.

1. Are there any controls or indicators located in positions that you found to be awkward or hard to reach? If so, where would you suggest that they be relocated?

A. (a) Move the Site status Summary Display down to the panel. Nothing causes the Operator to direct his attention to its location. (b) Move the CCTV Facilities at Selcted zones more to the center of the console. Nothing causes the operator to direct his attention to them. (1) A character generator could be used to display the affected zone on the monitor and eliminate the other indicators.

B. Site status lights should be lower on the console. CCTV equipment lights should be closer to the center of the console.

C. Move the systems display down so that it was more visible to the Operator, and move the CCTV indicators to a position inside the zone screens so that they could be more easily detected. This would cut down on response time when 
the Operator is assessing a situation in a particular zone. I also believe that I would move the camera control handle. In its present location it inhibits the movement of one's hand from the key pad to the select zone button.

D. The Site Status Summary Display where it is located now does not attract the operator's attention. Those summary lights should be lowered to get more attention. Same should be done for date and time displays. In reference to the CCTV display lights and zone indicator (located next to TVs on outside) should be moved in between the 2 TVs on both sides. While working controls you hve no occasion to look at lights. If moved between TVs much more useful for operators. The camera control knob should be moved up away from edge of panel. It makes it awkward to move hands and is easily leaned on there for moving camera unintentionally.

E. The zone CCTV lights on the side of the panel should be moved in towards the center. Reason being that usually there is no reason to look that far off to the side. Also the camera pan handle should be moved up about six inches so it wont inhibit the movement of your hands from side to the other. The functions lights at the top should be moved to the control panel itself because it is very hard to be aware of it when it's way up top.

2. Did you find the labeling of any of the controls or displays confusing or hard to understand? If so, how do you thing it should be changed?

A. Labeling of the controls and indicators seemed to be useful in the understanding and operation of the console. $\mathrm{HQ}$ data link is confusing unless it is fully explained, i.e., what $\mathrm{HQ}$, where it's located, and what type link you are talking about. There are no indicators to let the operator know his equipment is working or when it fails.

B. NO.

C. Labeling quite satisfactory. Console is uncluttered by unnecessary labels, making controls easy to locate. CCTV indicator lights could be brighter.

D. Lights on indicators should be brighter, especially the green lights. Lights should not have smoke colored cover over them. It should be clear, especially the "no camera" light.

E. Some lights should be brighter.

3. Did you find it easy or difficult to learn to use the 
console? If so, describe what the problem was.

A. Easy to understand and work with.

B. Easy to learn.

C. Easy to use.

D. Very easy to use.

E. A challenge, but not very hard to learn.

4. What is your opinion about the usefulness of the Monitor pointing switch for stepping through a group of uncleared alarms?

A. It's big and noisey.

B. Switch makes it possible to see all alarms very quickly and take action for all alarms.

C. Pointing switch extremely useful. Seems to be one of the fundamental and essential features of the system. Use of the switch allows the operator to pull all information on a particular zone instantaneously without choosing zones that have previously been selected. The operator would undoubtedly need this capability in the event that multiple incidents would take place.

D. Moving point was very useful in stepping through alarms. Switch made it possible for operator to go through alarms more quickly and get an idea of how things are going on the site.

E. It is all right.

5. When using the Monitor pointing Switch, did you have any difficulty in keeping track of the zone to which your controls were attached? If so, what would make it easier for you to keep track of which zone the controls are curently associated with?

A. Didn't find it difficult to keep track of.

B. No problems with it.

C. No problem keeping track of zones. Any problems could be remedied by moving the CCTV indicators in so that he had 3 quick reference points close to the center of the screen indicating what zone he's in at a given moment. 
D. No problem keeping track of zones. If you move the CCTV equipment in between the 2 TVs you would have 3 items telling him which zone he was in.

E. When you switch the lever over in a 2 or more alarm situation, the computer should say, for example, vibration alarm zone 2 and when you switch over to the second alarm, it should specify zone and circumstances.

6. Did you find the Pan/Tilt zoom control easy to use? If not, what arrangement of controls for these functions do you think would have been better?

A. Easy to use but compared to other controls and indicators is large and bulky.

B. Easy to use

C. Easy; no rearrángement necessary.

D. Easy to use. Should be moved up on console.

E. Easy to use, but should be moved up about 6 inches.

7. Are there any other controls or displays you think should be on the console? If you answer yes, please describe the form and function of these additional controls and/or displays.

A. Some type of indicator that would indicate the time remaining on the electric key. Some type of indicator that will show that a TV camera has gone bad.

B. Would like to test all sensors and feds at once.

C. TV bad indicator. A 60 second count down after sent key and a time remaining indicator for keys.

D. Camera down zone. Key code countdown. A 60 second count down display.

E. Detailed map board above the system with led lights so when someone goes out and calls in we know exactly where they are and can assist them by giving them the most direct route possible.

8. Are there any controls or displays on the console that you feel could be removed? If so, which ones, and why do you think that they should be removed?

A. Difficult to say. 
B. No.

C. No.

D. No.

E. No.

9. Did you find the console to be intimidating, or friendly
to learn to use?

A. Easy to use.

B. Easy to understand.

C. Friendly. Overloading an operator can be a problem with any security sys'em

D. Very easy to use.

E. Friendly

10. Do you think that this sort of simulator would be a useful tool for training personnel to work with a future fielc model Computerized site Security Monitor and Response system?

A. As a training simulator, it should be set up where the computers could control more than one console. This would clit down on the cost and increase the number of students that could be in training at one time. Preprogram scenarios so the trainer doesn't lose track of what has taken place.

B. Yes, if the simulation was improved.

C. Yes, but the simulation would need a great deal of improvement to include the geographical display unit, video type (instead of still pictures) and scenarios

D. Yes, but computer should be programmed to do everything the terrorist terminal does. Have precomputerized scenarios. Should have video tapes camera pictures.

E. Yes. They should have a school or even a different MOS for operating this unit. Someone should have a basic knowledge of computers and how they work before they operate on a reaI ADA site.

11. Please continue with any other comments that you wish to make. 
A. (1) Sensor test all at once rather than individually. (2) Software to indicate the number of times a sensor has been activated. (3) Eliminate the voice announcement of the operator change and their code. (4) Ability to shut down sensors for maintenance or due to high nuisance or false alarm rate. (5) Have a portion of the computer that could be programmed locally to indicate local instructions for the operator to follow. (6) Green lights need to be brighter. (7) Time for notification of $\mathrm{HQ}$ that an operator has failed to respond to an alarm is too long. (8) FEDS are a use of force and should be dealt with the same as the use of a weapon, K-9, nightstick, or MACE. (9) Remove the term "Guard." (10) Stop considering the military police and security police as dumb. The operator of this type of equipment will be highly motivated and educated to the point that he will be able to understand technical information.

B. None.

C. My only comments address the human element and not the system itself. First, I would want to include an exterior check and balance system so that the operators can be monitored, making sure they are doing their jobs and responding to situations in an acceptable time span. Also for the sake of security and integrity of the entire system, I would implement a couse of training for use of system to include a "deadly force" type rule for the use of FEDS, and realistic simulations so that the operator is completely aware of his responsibilities. Upon completion of the couse, the individual would be certified to operate the system and only those that are certified may do so. This could help in eliminating misuse of the system. I don't see the system as one that should be operated by just anyone.

D. The console is a pleasure to work with and would really be useful in actual environment. Having just left a hawk missile tactical site in Germany I can see where it would fit in and work exceptionally well.

E. I never had any real experience with computers but I had no problem understanding principles behind the system.

12. Education:

A. A/A in Police Science. Total of 95 semester hours in occupational education and computer programming.

B. High school diploma. Private pilot. Worked as technician with T.C.I. for three years.

C. High school and two years college in fine arts and 
business administration. Former art director and department head for graphic arts company.

D. High school graduate. Civilian police desk operations and controls. Milirary police school graduate.

E. High school graduate. 
NBS.IILA (REV. 2-80)

U.S. DEPT. OF COMM.

BIBLIOGRAPHIC DATA

SHEET (See instructions)

1. PUBLICATION OR

2. Performing Organ. Report Nod 3. Publication Date REPORT NO.

NBSIR $82-2656$

February 1983

4. $7 !$ ITLE AND SUBTITLE

Simulation of the Guard Control Station in a Computerized Site Security

Monitor and Response System

5. AUTHOR(S)

R.T. Moore, A.W. Holt, A.L. Koenig, A. Mink, G. Nacht

6. PERFORMING ORGANIZATION (If joint or other than NBS, see instructions)

NATIONAL BUREAU OF STANDARDS

DEPARTMENT OF COMMERCE

WASHINGTON, D.C. 20234

9. SPONSORING ORGANIZATION NAME AND COMPLETE ADDRESS (Street, City, State, ZIP)

Defense Nuclear Agency Task Code B99QAXRF

Wash.,D.C. 20305

Work Unit 00014

10. SUPPLEMENTARY NOTES

[ Document describes a computer program; SF-185, FIPS Software Summary, is attached.

11. ABSTRACT (A 200-word or less factual summary of most significant information. If document includes a significant bibliography or literature survey. mention it here)

This report describes a mock-up of a Guard Control Station that was used in simulating the performance of this component of a Computerized Site Security Monitor and Response System. The mock-up was interconnected to three microcomputer systems in an arrangement that permitted simulation of physical security scenarios in an interactive mode. Seven different individuals were allowed to act as operators of the Guard Control Station Console following a brief period of training. Based both on observations of their actions and on their comments, it was determined that the CSSMRS concepts were effective and easy to learn. The Console was judged to have met most design objectives, but certain controls and displays were identified as candidates for minor modification or relocation to further improve the console's overall effectiveness.

12. KEY WORDS (Six to twelve entries; alphabetical order: capitalize only proper names; and separate key words by semicolons)

13. AVAILABILITY

XX Unlimited

$\square$ For Official Distribution. Do Not Release to NTIS

$\square$ Order From Superintendent of Documents, U.S. Government Printing Office, Washington, D.C. 20402.

XX Order From National Technical Information Service (NTIS), Springfield, VA. 2216I
14. NO. OF

PRINTED PAGES

105

15. Price

$\$ 13.00$ 


